Supplementary Materials for

\title{
Suitability of 17 rainfall and temperature gridded datasets for large-scale hydrological modelling in West Africa
}

Moctar Dembélé ${ }^{1}$, Bettina Schaefli ${ }^{1,3}$, Nick van de Giesen $^{2} \&$ Grégoire Mariéthoz ${ }^{1}$

${ }^{1}$ Institute of Earth Surface Dynamics, Faculty of Geosciences and Environment, University of Lausanne, CH-1015 Lausanne, Switzerland

${ }^{2}$ Water Resources Section, Faculty of Civil Engineering and Geosciences, Delft University of Technology, Stevinweg 1, 2628 CN Delft, The Netherlands

${ }^{3}$ Now at: Institute of Geography, Faculty of Science, University of Bern, CH-3012, Switzerland

Correspondence to: Moctar Dembélé (moctar.dembele@unil.ch; mocdembele@gmail.com)

\section{Content}

This supplementary materials file contains additional figures and tables to support the analysis of the results presented in the main manuscript. The methodology used to obtain these results is explained in the main manuscript.

The following sections are presented:

- Section 2: Spatial long-term averages of rainfall and temperature Figure S1 to Figure S2

- Section 3: Temporal long-term averages of rainfall and temperature Figure S3 to Figure S6

- Section 4: Ranking of rainfall and temperature datasets Figure $\mathrm{S} 7$

- Section 5: Model performance for streamflow Figure S8 to Figure S12; Table S1 and Table S12

- Section 6: Model performance for terrestrial water storage Figure S13 to Figure S28; Table S13

- Section 7: Model performance for soil moisture Figure S29 to Figure S46; Table S14 to Table S15

- Section 8: Model performance for evaporation Figure S47 to Figure S64; Table S16 to Table S17

- Section 9: Model parameters

Figure S65 to Figure S66; Table S18 
2 Spatial long-term averages of rainfall and temperature

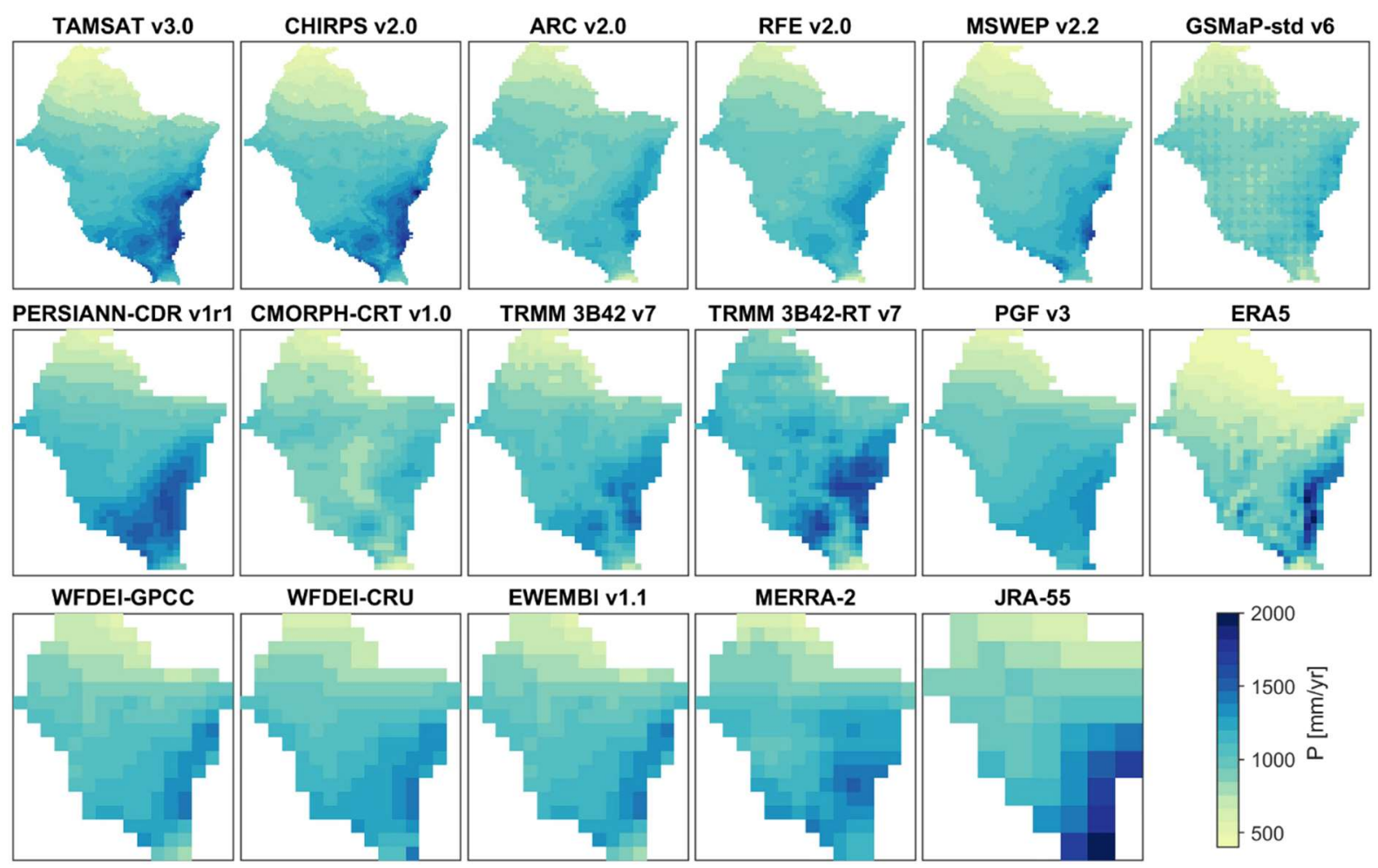

Figure S1. Mean annual rainfall totals over the period 2003-2012 for 17 rainfall datasets the Volta River basin

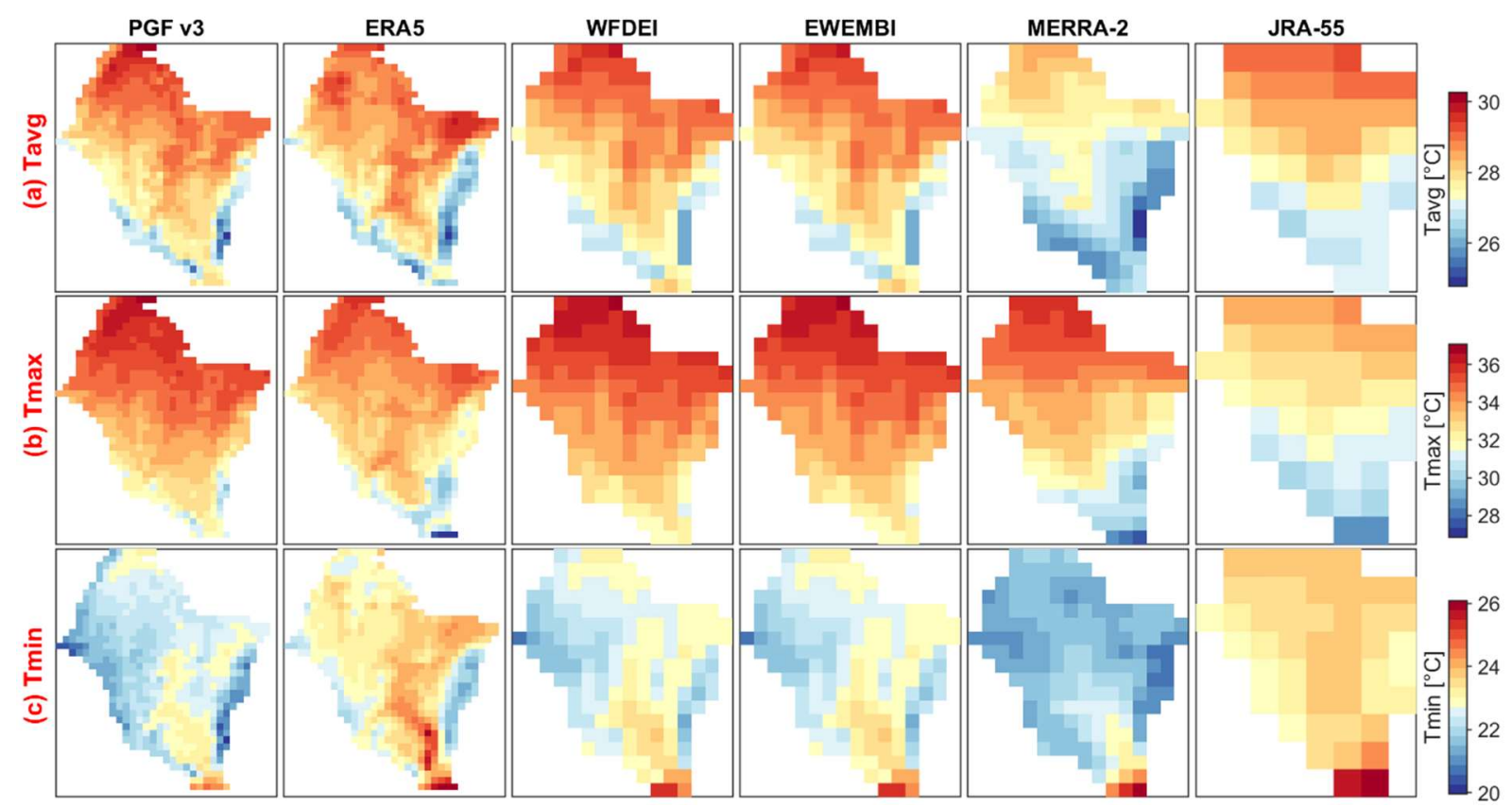

Figure S2. Mean annual air temperature (average (a), maximum (b) and minimum (c)) over the period 2003-2012 for 6 temperature datasets in the Volta River basin 
3 Temporal long-term averages of rainfall and temperature

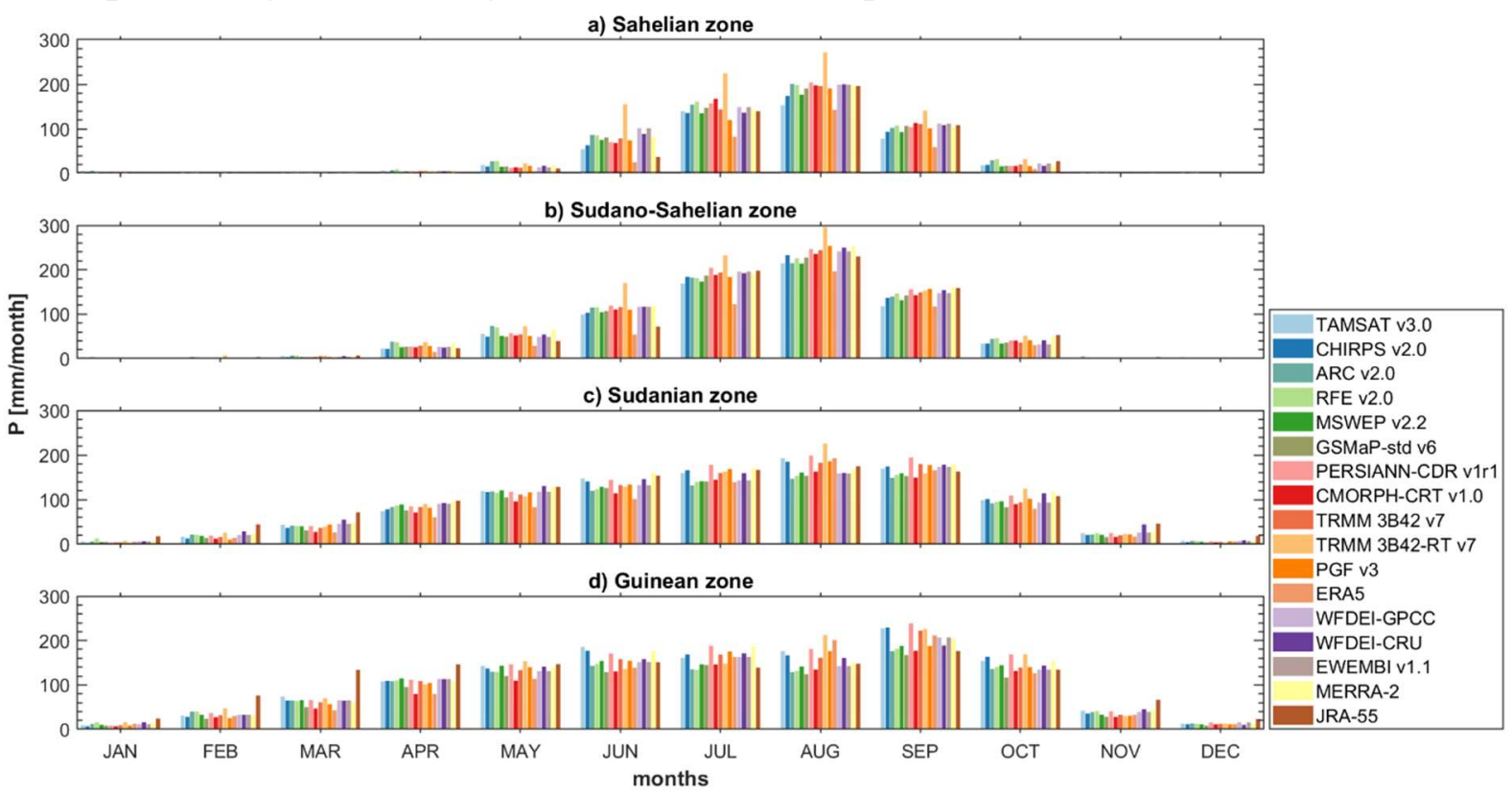

Figure S3. Climatology of mean monthly rainfall totals over the period 2003-2012, averaged over four climatic zones ( $\mathrm{a}, \mathrm{b}, \mathrm{c}$ and $\mathrm{d})$. . The colored bars represent 17 rainfall datasets.
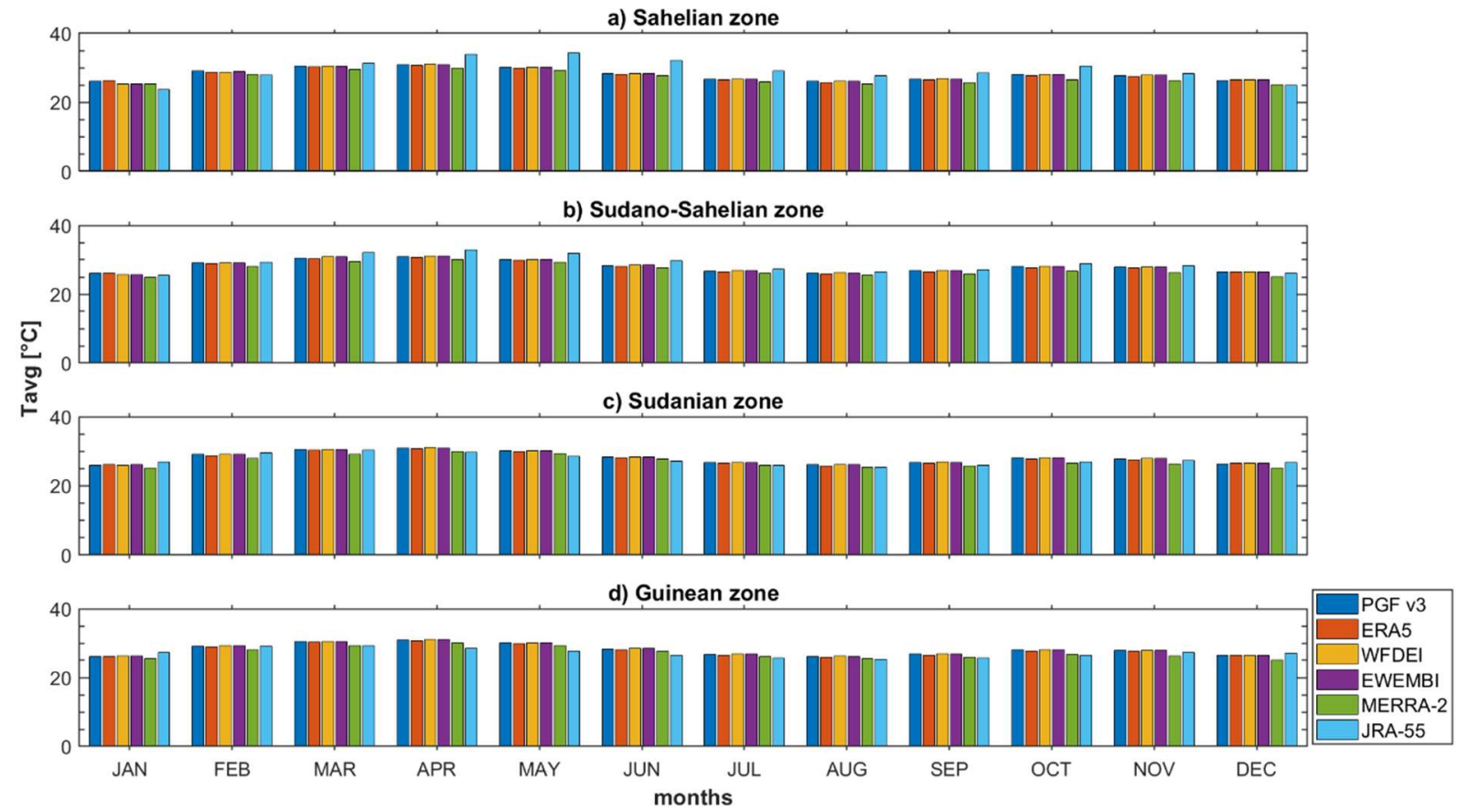

Figure S4. Climatology of mean monthly average air temperature over the period 2003-2012, averaged over four climatic zones ( $\mathrm{a}, \mathrm{b}, \mathrm{c}$ and $\mathrm{d})$. The colored bars represent 6 temperature datasets. 

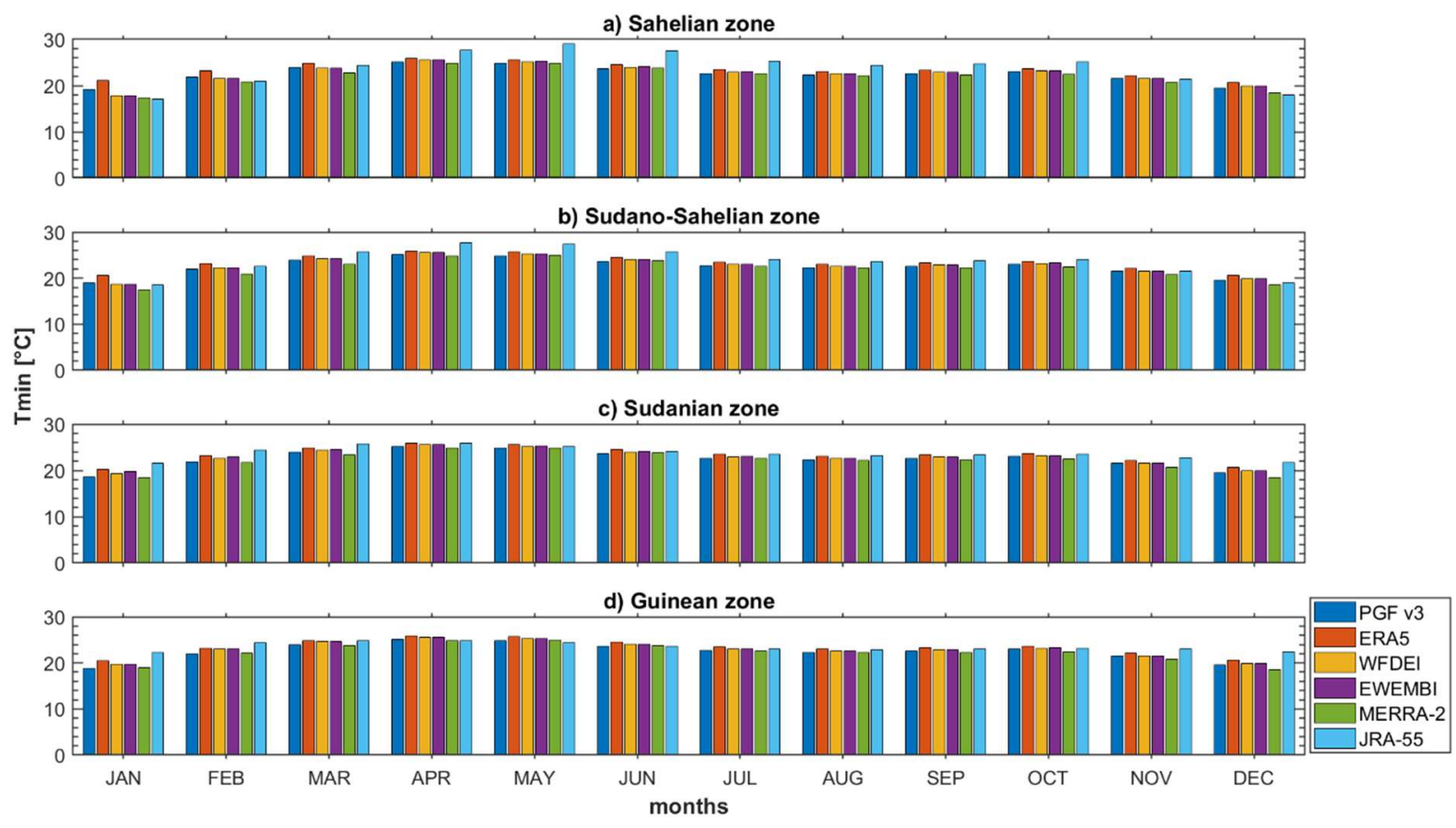

Figure S5. Climatology of mean monthly minimum air temperature over the period 2003-2012, averaged over four climatic zones ( $\mathrm{a}, \mathrm{b}, \mathrm{c}$ and $\mathrm{d})$. The colored bars represent 6 temperature datasets.
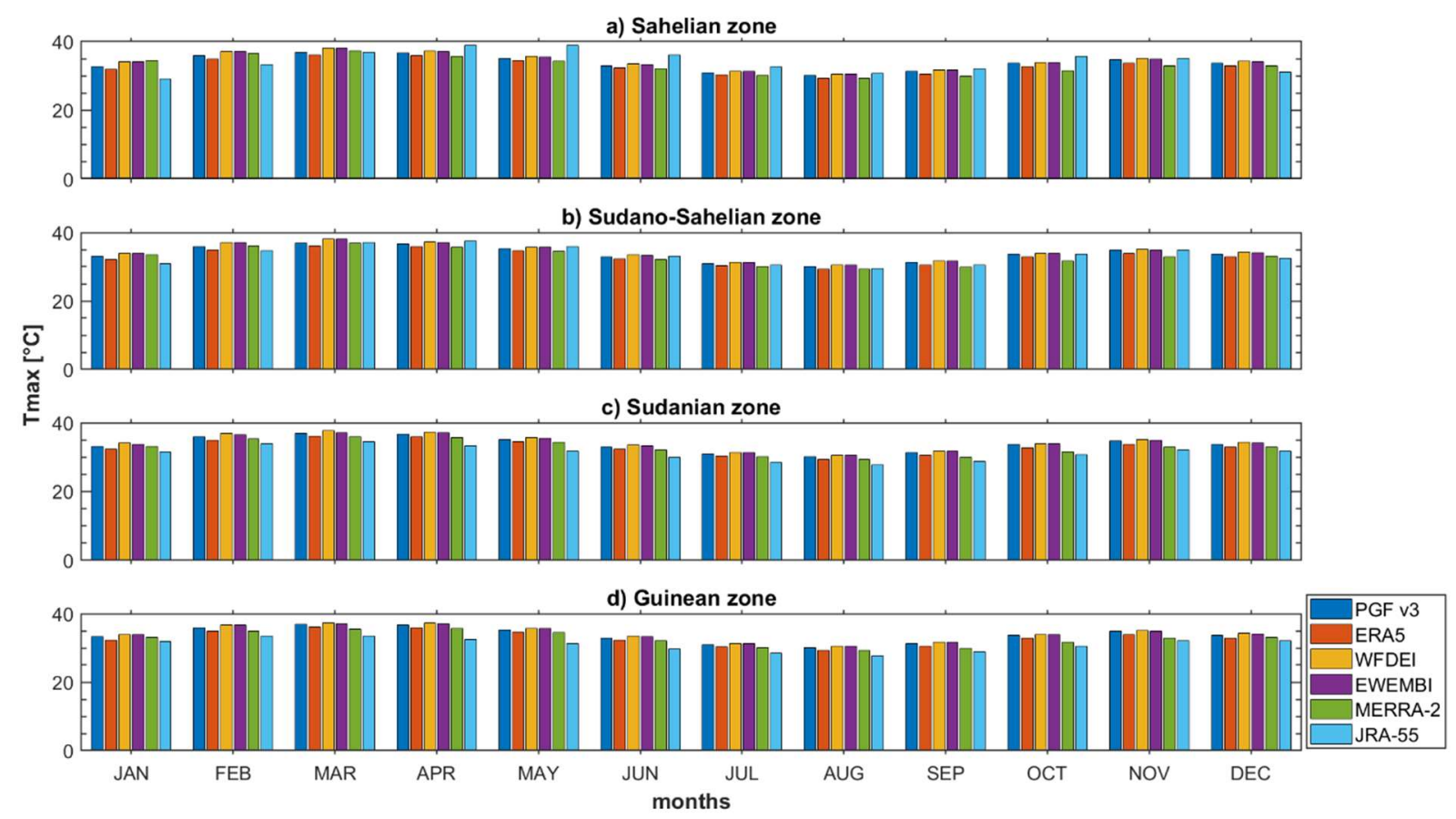

Figure S6. Climatology of mean monthly maximum air temperature over the period 2003-2012, averaged over four climatic zones ( $\mathrm{a}, \mathrm{b}, \mathrm{c}$ and $\mathrm{d})$. The colored bars represent 6 temperature datasets. 
4 Ranking of rainfall and temperature datasets

\begin{tabular}{|c|c|c|c|c|c|c|}
\hline & & \multicolumn{5}{|c|}{ Top 5 rainfall datasets for a given temperature dataset } \\
\hline & & 1 & 2 & 3 & 4 & 5 \\
\hline & & \multicolumn{5}{|c|}{ Temporal dynamics of $Q$} \\
\hline \multirow{41}{*}{ 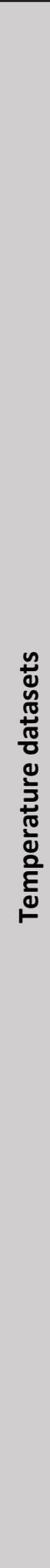 } & JRA-55 & TAMSAT & GSMaP-std & CHIRPS & MERRA-2 & CMORPH-CRT \\
\hline & MERRA-2 & TAMSAT & GSMaP-std & PERSIANN-CDR & CHIRPS & CMORPH-CRT \\
\hline & EWEMBI & TAMSAT & GSMaP-std & CHIRPS & MERRA-2 & ARC \\
\hline & WFDEI & TAMSAT & GSMaP-std & CHIRPS & MERRA-2 & ARC \\
\hline & ERA5 & CHIRPS & TAMSAT & CMORPH-CRT & GSMaP-std & ARC \\
\hline & PGF v3 & TAMSAT & GSMaP-std & PERSIANN-CDR & CHIRPS & MERRA-2 \\
\hline & & \multicolumn{5}{|c|}{ Temporal dynamics of $S t$} \\
\hline & JRA-55 & CMORPH-CRT & ARC & RFE & TAMSAT & CHIRPS \\
\hline & MERRA-2 & ARC & RFE & TRMM 3B42-RT & WFDEI-CRU & CHIRPS \\
\hline & EWEMBI & ARC & CHIRPS & TRMM 3B42-RT & GSMaP-std & ERA5 \\
\hline & WFDEI & ARC & CHIRPS & TRMM 3B42-RT & GSMaP-std & ERA5 \\
\hline & ERA5 & ARC & RFE & CMORPH-CRT & TAMSAT & TRMM 3B42-RT \\
\hline & PGF v3 & CMORPH-CRT & RFE & ARC & CHIRPS & TAMSAT \\
\hline & & \multicolumn{5}{|c|}{ Temporal dynamics of Su } \\
\hline & JRA-55 & WFDEI-GPCC & EWEMBI & MERRA-2 & PGF & TAMSAT \\
\hline & MERRA-2 & MERRA-2 & WFDEI-GPCC & EWEMBI & PERSIANN-CDR & ERA5 \\
\hline & EWEMBI & WFDEI-CRU & PGF & PERSIANN-CDR & MERRA-2 & GSMaP-std \\
\hline & WFDEI & WFDEI-CRU & PGF & PERSIANN-CDR & MERRA-2 & GSMaP-std \\
\hline & ERA5 & WFDEI-GPCC & EWEMBI & MERRA-2 & PGF & ERA5 \\
\hline & PGF v3 & WFDEI-GPCC & EWEMBI & WFDEI-CRU & MERRA-2 & TAMSAT \\
\hline & & \multicolumn{5}{|c|}{ Temporal dynamics of $E a$} \\
\hline & JRA-55 & ARC & RFE & TAMSAT & WFDEI-GPCC & EWEMBI \\
\hline & MERRA-2 & ARC & CMORPH-CRT & RFE & WFDEI-GPCC & EWEMBI \\
\hline & EWEMBI & RFE & GSMaP-std & ARC & TAMSAT & PERSIANN-CDR \\
\hline & WFDEI & RFE & GSMaP-std & ARC & TAMSAT & PERSIANN-CDR \\
\hline & ERA5 & ARC & RFE & WFDEI-GPCC & EWEMBI & GSMaP-std \\
\hline & PGF v3 & TAMSAT & WFDEI-GPCC & EWEMBI & WFDEI-CRU & ARC \\
\hline & & \multicolumn{5}{|c|}{ Spatial patterns of $S u$} \\
\hline & JRA-55 & WFDEI-GPCC & EWEMBI & TAMSAT & MSWEP & RFE \\
\hline & MERRA-2 & MSWEP & PGF & ARC & WFDEI-CRU & WFDEI-GPCC \\
\hline & EWEMBI & TAMSAT & PERSIANN-CDR & MSWEP & WFDEI-GPCC & EWEMBI \\
\hline & WFDEI & TAMSAT & PERSIANN-CDR & MSWEP & WFDEI-GPCC & EWEMBI \\
\hline & ERA5 & MSWEP & RFE & WFDEI-GPCC & EWEMBI & WFDEI-CRU \\
\hline & PGF v3 & RFE & TAMSAT & MSWEP & ARC & MERRA-2 \\
\hline & & \multicolumn{5}{|c|}{ Spatial patterns of $E a$} \\
\hline & JRA-55 & TAMSAT & WFDEI-GPCC & EWEMBI & MSWEP & MERRA-2 \\
\hline & MERRA-2 & MSWEP & CHIRPS & MERRA-2 & WFDEI-CRU & PGF \\
\hline & EWEMBI & PERSIANN-CDR & TAMSAT & MSWEP & WFDEI-GPCC & EWEMBI \\
\hline & WFDEI & PERSIANN-CDR & TAMSAT & MSWEP & WFDEI-GPCC & EWEMBI \\
\hline & ERA5 & MSWEP & MERRA-2 & WFDEI-GPCC & EWEMBI & PERSIANN-CDR \\
\hline & PGF v3 & MSWEP & CHIRPS & TAMSAT & MERRA-2 & PERSIANN-CDR \\
\hline
\end{tabular}

Figure S7. Best rainfall-temperature dataset combinations for simulating the spatial patterns and the temporal dynamics of streamflow $(Q)$, terrestrial water storage $\left(S_{t}\right)$, soil moisture $\left(S_{\mathrm{u}}\right)$ and actual evaporation $\left(E_{\mathrm{a}}\right)$ in the Volta River basin. 


\section{Model performance for streamflow}

\subsection{Ranking of rainfall and temperature datasets}

\subsubsection{Combined calibration and evaluation periods}

Table S1. Ranking of rainfall and temperature datasets based on median Kling-Gupta efficiency $\left(E_{K G}\right)$ of streamflow $(Q)$ over the simulation period. The mean and the second-order coefficient of variation $\left(V_{2}\right)$ are calculated across 6 temperature datasets for each rainfall dataset, and across 17 rainfall datasets for each temperature dataset. The rank is given from the best (green) to the worst performance (red) for the Volta River basin (VRB)

\begin{tabular}{|c|c|c|c|c|c|c|c|c|c|c|c|}
\hline \multirow{2}{*}{\multicolumn{2}{|c|}{$\begin{array}{c}\text { Kling-Gupta Efficiency of Q } \\
\text { Simulation period } \\
\text { 2003-2012 }\end{array}$}} & \multicolumn{6}{|c|}{ Temperature datasets } & \multicolumn{4}{|c|}{ Statistics across temperature } \\
\hline & & JRA-55 & MERRA-2 & EWEMBI & WFDEI & ERA5 & PGF v3 & Mean & $V_{2}(\%)$ & $\begin{array}{l}\text { Rank - } \\
\text { on mean }\end{array}$ & $\begin{array}{l}\text { Rank - } \\
\text { on } V_{2}\end{array}$ \\
\hline \multirow{17}{*}{ 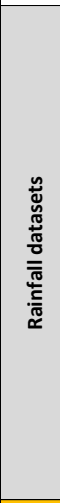 } & TAMSAT V3.0 & 0.727 & 0.771 & 0.719 & 0.719 & 0.718 & 0.730 & 0.73 & 2.81 & 1 & 16 \\
\hline & CHIRPS v2.0 & 0.696 & 0.700 & 0.708 & 0.708 & 0.726 & 0.683 & 0.70 & 2.03 & 3 & 8 \\
\hline & ARC v2.0 & 0.677 & 0.656 & 0.686 & 0.686 & 0.698 & 0.679 & 0.68 & 2.04 & 7 & 9 \\
\hline & RFE v2.0 & 0.635 & 0.624 & 0.658 & 0.658 & 0.663 & 0.641 & 0.65 & 2.43 & 11 & 13 \\
\hline & MSWEP v2.2 & 0.661 & 0.666 & 0.653 & 0.653 & 0.640 & 0.661 & 0.66 & 1.39 & 10 & 3 \\
\hline & GSMaP-std v6 & 0.717 & 0.715 & 0.708 & 0.708 & 0.717 & 0.714 & 0.71 & 0.54 & 2 & 1 \\
\hline & PERSIANN-CDR v1r1 & 0.678 & 0.704 & 0.667 & 0.667 & 0.683 & 0.689 & 0.68 & 2.08 & 6 & 10 \\
\hline & CMORPH-CRT v1.0 & 0.683 & 0.695 & 0.684 & 0.684 & 0.717 & 0.673 & 0.69 & 2.21 & 4 & 11 \\
\hline & TRMM 3B42 v7 & 0.524 & 0.563 & 0.543 & 0.543 & 0.524 & 0.535 & 0.54 & 2.73 & 16 & 15 \\
\hline & TRMM 3B42-RT v7 & 0.543 & 0.542 & 0.544 & 0.544 & 0.521 & 0.545 & 0.54 & 1.72 & 15 & 4 \\
\hline & PGF v3 & 0.664 & 0.686 & 0.652 & 0.652 & 0.664 & 0.650 & 0.66 & 2.02 & 9 & 7 \\
\hline & ERA5 & 0.604 & 0.643 & 0.641 & 0.641 & 0.620 & 0.617 & 0.63 & 2.62 & 14 & 14 \\
\hline & WFDEI-GPCC & 0.642 & 0.659 & 0.631 & 0.631 & 0.634 & 0.629 & 0.64 & 1.76 & 12 & 5 \\
\hline & WFDEI-CRU & 0.656 & 0.692 & 0.652 & 0.652 & 0.674 & 0.669 & 0.67 & 2.40 & 8 & 12 \\
\hline & EWEMBI v1.1 & 0.642 & 0.659 & 0.631 & 0.631 & 0.634 & 0.629 & 0.64 & 1.76 & 12 & 5 \\
\hline & \begin{tabular}{|l|} 
MERRA-2 \\
\end{tabular} & 0.692 & 0.687 & 0.688 & 0.688 & 0.667 & 0.683 & 0.68 & 1.30 & 5 & 2 \\
\hline & JRA-55 & 0.439 & 0.487 & 0.443 & 0.443 & 0.451 & 0.457 & 0.45 & 3.87 & 17 & 17 \\
\hline \multirow{4}{*}{ 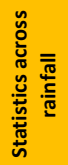 } & Mean & 0.64 & 0.66 & 0.64 & 0.64 & 0.64 & 0.64 & & & & \\
\hline & $V_{2}(\%)$ & 11.6 & 10.5 & 11.0 & 11.0 & 12.0 & 10.8 & & & & \\
\hline & Rank - on mean & 6 & 1 & 4 & 3 & 2 & 5 & & & & \\
\hline & Rank - on V2 & 5 & 1 & 4 & 3 & 6 & 2 & & & & \\
\hline
\end{tabular}

Table S2. Ranking of rainfall and temperature datasets based on median Pearson correlation coefficient $(r)$ of streamflow $(Q)$ over the simulation period. The mean and the second-order coefficient of variation $\left(V_{2}\right)$ are calculated across 6 temperature datasets for each rainfall dataset, and across 17 rainfall datasets for each temperature dataset. The rank is given from the best (green) to the worst performance (red) for the Volta River basin (VRB)

\begin{tabular}{|c|c|c|c|c|c|c|c|c|c|c|c|}
\hline \multirow{2}{*}{\multicolumn{2}{|c|}{$\begin{array}{c}\text { Correlation of Q } \\
\text { Simulation period } \\
\text { 2003-2012 }\end{array}$}} & \multicolumn{6}{|c|}{ Temperature datasets } & \multicolumn{4}{|c|}{ Statistics across temperature } \\
\hline & & JRA-55 & MERRA-2 & EWEMBI & WFDEI & ERA5 & PGF v3 & Mean & V2 (\%) & $\begin{array}{c}\text { Rank - } \\
\text { on mean }\end{array}$ & $\begin{array}{l}\text { Rank - } \\
\text { on } V_{2}\end{array}$ \\
\hline \multirow{17}{*}{ 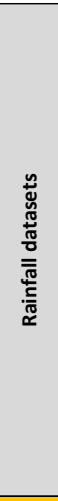 } & TAMSAT V3.0 & 0.907 & 0.904 & 0.907 & 0.907 & 0.898 & 0.906 & 0.90 & 0.40 & 4 & 10 \\
\hline & CHIRPS v2.0 & 0.912 & 0.906 & 0.909 & 0.909 & 0.913 & 0.913 & 0.91 & 0.33 & 1 & 6 \\
\hline & ARC v2.0 & 0.908 & 0.907 & 0.910 & 0.910 & 0.911 & 0.910 & 0.91 & 0.18 & 2 & 2 \\
\hline & RFE v2.0 & 0.906 & 0.901 & 0.911 & 0.911 & 0.902 & 0.903 & 0.91 & 0.48 & 3 & 15 \\
\hline & MSWEP v2.2 & 0.889 & 0.883 & 0.893 & 0.893 & 0.889 & 0.893 & 0.89 & 0.46 & 11 & 14 \\
\hline & GSMaP-std v6 & 0.898 & 0.900 & 0.898 & 0.898 & 0.900 & 0.898 & 0.90 & 0.12 & 8 & 1 \\
\hline & PERSIANN-CDR V1r1 & 0.906 & 0.899 & 0.902 & 0.902 & 0.906 & 0.903 & 0.90 & 0.32 & 5 & 4 \\
\hline & CMORPH-CRT v1.0 & 0.890 & 0.896 & 0.908 & 0.908 & 0.903 & 0.887 & 0.90 & 1.00 & 7 & 17 \\
\hline & TRMM 3B42 v7 & 0.883 & 0.888 & 0.893 & 0.893 & 0.886 & 0.891 & 0.89 & 0.46 & 14 & 13 \\
\hline & TRMM 3B42-RT V7 & 0.892 & 0.891 & 0.893 & 0.893 & 0.886 & 0.890 & 0.89 & 0.32 & 10 & 5 \\
\hline & PGF v3 & 0.895 & 0.901 & 0.897 & 0.897 & 0.897 & 0.892 & 0.90 & 0.34 & 9 & 7 \\
\hline & ERA5 & 0.844 & 0.851 & 0.846 & 0.846 & 0.848 & 0.842 & 0.85 & 0.36 & 17 & 8 \\
\hline & WFDEI-GPCC & 0.887 & 0.887 & 0.894 & 0.894 & 0.891 & 0.886 & 0.89 & 0.42 & 12 & 11 \\
\hline & WFDEI-CRU & 0.870 & 0.885 & 0.871 & 0.871 & 0.878 & 0.885 & 0.88 & 0.82 & 15 & 16 \\
\hline & EWEMBI v1.1 & 0.887 & 0.887 & 0.894 & 0.894 & 0.891 & 0.886 & 0.89 & 0.42 & 12 & 11 \\
\hline & MERRA-2 & 0.900 & 0.895 & 0.901 & 0.901 & 0.897 & 0.901 & 0.90 & 0.27 & 6 & 3 \\
\hline & JRA-55 & 0.855 & 0.862 & 0.856 & 0.856 & 0.856 & 0.862 & 0.86 & 0.40 & 16 & 9 \\
\hline \multirow{4}{*}{ 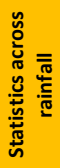 } & Mean & 0.89 & 0.89 & 0.89 & 0.89 & 0.89 & 0.89 & & & & \\
\hline & $V_{2}(\%)$ & 2.1 & 1.7 & 2.1 & 2.1 & 2.0 & 2.0 & & & & \\
\hline & Rank - on mean & 6 & 5 & 2 & 1 & 3 & 4 & & & & \\
\hline & Rank - on V/2 & 6 & 1 & 4 & 5 & 3 & 2 & & & & \\
\hline
\end{tabular}


Table S3. Ranking of rainfall and temperature datasets based on median bias $(\beta)$ of streamflow $(Q)$ over the simulation period. The mean and the second-order coefficient of variation $\left(V_{2}\right)$ are calculated across 6 temperature datasets for each rainfall dataset, and across 17 rainfall datasets for each temperature dataset. The rank is given from the best (green) to the worst performance (red) for the Volta River basin (VRB)

\begin{tabular}{|c|c|c|c|c|c|c|c|c|c|c|c|}
\hline \multirow{2}{*}{\multicolumn{2}{|c|}{$\begin{array}{c}\text { Bias of Q } \\
\text { Simulation period } \\
\text { 2003-2012 }\end{array}$}} & \multicolumn{6}{|c|}{ Temperature datasets } & \multicolumn{4}{|c|}{ Statistics across temperature } \\
\hline & & JRA-55 & MERRA-2 & EWEMBI & WFDEI & ERA5 & PGF v3 & Mean & $V_{2}(\%)$ & $\begin{array}{c}\text { Rank - } \\
\text { on mean }\end{array}$ & $\begin{array}{l}\text { Rank - } \\
\text { on } V_{2}\end{array}$ \\
\hline \multirow{17}{*}{ 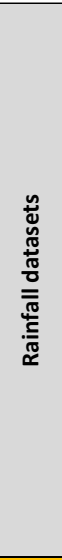 } & TAMSAT v3.0 & 0.865 & 0.911 & 0.846 & 0.846 & 0.880 & 0.855 & 0.87 & 2.86 & 7 & 13 \\
\hline & CHIRPS V2.0 & 0.829 & 0.847 & 0.837 & 0.837 & 0.849 & 0.829 & 0.84 & 1.03 & 8 & 3 \\
\hline & ARC v2.0 & 0.979 & 0.987 & 0.982 & 0.982 & 1.012 & 0.988 & 0.99 & 1.22 & 2 & 7 \\
\hline & RFE v2.0 & 1.006 & 1.051 & 0.976 & 0.976 & 0.980 & 1.007 & 1.00 & 2.91 & 1 & 14 \\
\hline & MSWEP v2.2 & 0.829 & 0.822 & 0.829 & 0.829 & 0.832 & 0.838 & 0.83 & 0.65 & 9 & 1 \\
\hline & GSMaP-std v6 & 0.926 & 0.949 & 0.910 & 0.910 & 0.931 & 0.918 & 0.92 & 1.60 & 4 & 8 \\
\hline & PERSIANN-CDR v1r1 & 0.812 & 0.867 & 0.814 & 0.814 & 0.825 & 0.824 & 0.83 & 2.52 & 10 & 11 \\
\hline & CMORPH-CRT v1.0 & 0.931 & 0.950 & 0.936 & 0.936 & 0.918 & 0.928 & 0.93 & 1.12 & 3 & 6 \\
\hline & TRMM 3B42 v7 & 0.651 & 0.654 & 0.673 & 0.673 & 0.630 & 0.664 & 0.66 & 2.48 & 16 & 10 \\
\hline & TRMM 3B42-RT v7 & 0.701 & 0.743 & 0.701 & 0.701 & 0.646 & 0.706 & 0.70 & 4.43 & 15 & 16 \\
\hline & PGF v3 & 0.799 & 0.846 & 0.825 & 0.825 & 0.784 & 0.825 & 0.82 & 2.67 & 11 & 12 \\
\hline & ERA5 & 0.905 & 0.817 & 0.910 & 0.910 & 0.767 & 0.911 & 0.87 & 7.16 & 6 & 17 \\
\hline & WFDEI-GPCC & 0.790 & 0.787 & 0.785 & 0.785 & 0.780 & 0.766 & 0.78 & 1.10 & 13 & 4 \\
\hline & WFDEI-CRU & 0.886 & 0.898 & 0.873 & 0.873 & 0.852 & 0.871 & 0.88 & 1.75 & 5 & 9 \\
\hline & EWEMBI v1.1 & 0.790 & 0.787 & 0.785 & 0.785 & 0.780 & 0.766 & 0.78 & 1.10 & 13 & 4 \\
\hline & MERRA-2 & 0.812 & 0.820 & 0.800 & 0.800 & 0.803 & 0.805 & 0.81 & 0.96 & 12 & 2 \\
\hline & \begin{tabular}{|l|} 
JRA-55 \\
\end{tabular} & 0.510 & 0.556 & 0.527 & 0.527 & 0.508 & 0.526 & 0.53 & 3.26 & 17 & 15 \\
\hline \multirow{4}{*}{ 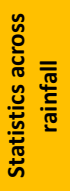 } & Mean & 0.82 & 0.84 & 0.82 & 0.82 & 0.81 & 0.82 & & & & \\
\hline & $V_{2}(\%)$ & 14.6 & 14.1 & 13.7 & 13.7 & 15.5 & 14.3 & & & & \\
\hline & Rank - on mean & 3 & 1 & 5 & 4 & 6 & 2 & & & & \\
\hline & Rank - on $\mathbf{V}_{2}$ & 5 & 3 & 1 & 2 & 6 & 4 & & & & \\
\hline
\end{tabular}

Table S4. Ranking of rainfall and temperature datasets based on median variability $(\gamma)$ of streamflow $(Q)$ over the simulation period. The mean and the second-order coefficient of variation $\left(V_{2}\right)$ are calculated across 6 temperature datasets for each rainfall dataset, and across 17 rainfall datasets for each temperature dataset. The rank is given from the best (green) to the worst performance (red) for the Volta River basin (VRB)

\begin{tabular}{|c|c|c|c|c|c|c|c|c|c|c|c|}
\hline \multirow{2}{*}{\multicolumn{2}{|c|}{$\begin{array}{c}\text { Variability of } Q \\
\text { Simulation period } \\
2003-2012\end{array}$}} & \multicolumn{6}{|c|}{ Temperature datasets } & \multicolumn{4}{|c|}{ Statistics across temperature } \\
\hline & & JRA-55 & MERRA-2 & EWEMBI & WFDEI & ERA5 & PGF v3 & Mean & $V_{2}(\%)$ & $\begin{array}{c}\text { Rank - } \\
\text { on mean }\end{array}$ & $\begin{array}{l}\text { Rank - } \\
\text { on } V_{2}\end{array}$ \\
\hline \multirow{17}{*}{ 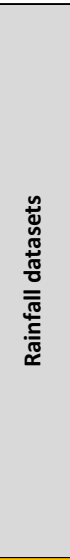 } & TAMSAT v3.0 & 0.953 & 0.963 & 0.970 & 0.970 & 0.911 & 0.968 & 0.96 & 2.40 & 11 & 15 \\
\hline & CHIRPS v2.0 & 0.989 & 0.984 & 0.998 & 0.998 & 0.959 & 0.977 & 0.98 & 1.50 & 2 & 9 \\
\hline & ARC v2.0 & 0.987 & 0.996 & 0.994 & 0.994 & 1.015 & 0.997 & 1.00 & 0.94 & 1 & 4 \\
\hline & RFE v2.0 & 0.964 & 0.964 & 0.953 & 0.953 & 0.969 & 0.981 & 0.96 & 1.08 & 10 & 5 \\
\hline & MSWEP V2.2 & 0.910 & 0.916 & 0.899 & 0.899 & 0.897 & 0.907 & 0.90 & 0.83 & 14 & 2 \\
\hline & GSMaP-std v6 & 0.962 & 0.993 & 0.987 & 0.987 & 0.976 & 0.979 & 0.98 & 1.11 & 4 & 6 \\
\hline & PERSIANN-CDR v1r1 & 0.979 & 0.927 & 0.982 & 0.982 & 0.963 & 0.963 & 0.97 & 2.19 & 9 & 13 \\
\hline & CMORPH-CRT v1.0 & 0.934 & 0.910 & 0.879 & 0.879 & 0.922 & 0.918 & 0.91 & 2.54 & 13 & 17 \\
\hline & TRMM 3B42 v7 & 0.836 & 0.845 & 0.803 & 0.803 & 0.836 & 0.802 & 0.82 & 2.44 & 17 & 16 \\
\hline & \begin{tabular}{|l|} 
TRMM 3B42-RT v7 \\
\end{tabular} & 0.848 & 0.842 & 0.825 & 0.825 & 0.841 & 0.826 & 0.83 & 1.25 & 16 & 7 \\
\hline & PGF v3 & 0.984 & 0.984 & 0.973 & 0.973 & 0.997 & 0.979 & 0.98 & 0.91 & 3 & 3 \\
\hline & ERA5 & 0.971 & 0.995 & 0.946 & 0.946 & 0.995 & 0.984 & 0.97 & 2.30 & 8 & 14 \\
\hline & WFDEI-GPCC & 0.995 & 0.995 & 0.954 & 0.954 & 0.984 & 0.984 & 0.98 & 1.95 & 6 & 10 \\
\hline & WFDEI-CRU & 0.986 & 0.981 & 0.973 & 0.973 & 0.998 & 0.960 & 0.98 & 1.32 & 5 & 8 \\
\hline & EWEMBI v1.1 & 0.995 & 0.995 & 0.954 & 0.954 & 0.984 & 0.984 & 0.98 & 1.95 & 6 & 10 \\
\hline & MERRA-2 & 0.949 & 0.948 & 0.950 & 0.950 & 0.960 & 0.959 & 0.95 & 0.57 & 12 & 1 \\
\hline & \begin{tabular}{|l|} 
JRA-55 \\
\end{tabular} & 0.872 & 0.849 & 0.845 & 0.845 & 0.867 & 0.888 & 0.86 & 2.03 & 15 & 12 \\
\hline \multirow{4}{*}{ 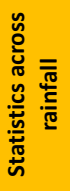 } & Mean & 0.95 & 0.95 & 0.93 & 0.93 & 0.95 & 0.94 & & & & \\
\hline & $V_{2}(\%)$ & 5.4 & 5.9 & 6.5 & 6.5 & 6.0 & 6.1 & & & & \\
\hline & Rank - on mean & 1 & 2 & 5 & 6 & 3 & 4 & & & & \\
\hline & Rank - on V $\mathbf{2}$ & 1 & 2 & 5 & 6 & 3 & 4 & & & & \\
\hline
\end{tabular}




\subsubsection{Separated calibration and evaluation periods}

Table S5. Ranking of rainfall and temperature datasets based on mean Kling-Gupta efficiency $\left(E_{K G}\right)$ of streamflow $(Q)$ over the calibration period. The mean and the second-order coefficient of variation $\left(V_{2}\right)$ are calculated across 6 temperature datasets for each rainfall dataset, and across 17 rainfall datasets for each temperature dataset. The rank is given from the best (green) to the worst performance (red) for the Volta River basin (VRB)

\begin{tabular}{|c|c|c|c|c|c|c|c|c|c|c|c|}
\hline \multirow{2}{*}{\multicolumn{2}{|c|}{$\begin{array}{c}\text { Kling-Gupta Efficiency of Q } \\
\text { Calibration period } \\
\text { 2003-2008 }\end{array}$}} & \multicolumn{6}{|c|}{ Temperature datasets } & \multicolumn{4}{|c|}{ Statistics across temperature } \\
\hline & & JRA-55 & MERRA-2 & EWEMBI & WFDEI & ERA5 & PGF v3 & Mean & $V_{2}(\%)$ & $\begin{array}{c}\text { Rank - } \\
\text { on mean }\end{array}$ & $\begin{array}{l}\text { Rank - } \\
\text { on } V_{2}\end{array}$ \\
\hline \multirow{17}{*}{ 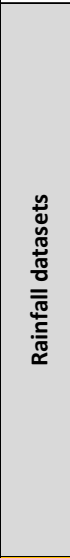 } & TAMSAT v3.0 & 0.702 & 0.728 & 0.704 & 0.704 & 0.701 & 0.708 & 0.71 & 1.43 & 1 & 16 \\
\hline & CHIRPS V2.0 & 0.660 & 0.674 & 0.672 & 0.672 & 0.674 & 0.666 & 0.67 & 0.83 & 5 & 7 \\
\hline & ARC v2.0 & 0.694 & 0.698 & 0.695 & 0.695 & 0.705 & 0.698 & 0.70 & 0.61 & 2 & 4 \\
\hline & RFE v2.0 & 0.672 & 0.666 & 0.657 & 0.657 & 0.678 & 0.666 & 0.67 & 1.27 & 6 & 15 \\
\hline & MSWEP v2.2 & 0.626 & 0.623 & 0.622 & 0.622 & 0.621 & 0.627 & 0.62 & 0.38 & 11 & 2 \\
\hline & GSMaP-std v6 & 0.667 & 0.681 & 0.678 & 0.678 & 0.677 & 0.676 & 0.68 & 0.71 & 3 & 5 \\
\hline & PERSIANN-CDR v1r1 & 0.673 & 0.672 & 0.671 & 0.671 & 0.669 & 0.670 & 0.67 & 0.22 & 4 & 1 \\
\hline & CMORPH-CRT V1.0 & 0.668 & 0.678 & 0.653 & 0.653 & 0.665 & 0.668 & 0.66 & 1.43 & 7 & 17 \\
\hline & TRMM 3B42 v7 & 0.525 & 0.537 & 0.538 & 0.538 & 0.530 & 0.532 & 0.53 & 1.04 & 17 & 12 \\
\hline & TRMM 3B42-RT v7 & 0.532 & 0.546 & 0.533 & 0.533 & 0.529 & 0.530 & 0.53 & 1.17 & 16 & 14 \\
\hline & PGF v3 & 0.640 & 0.660 & 0.650 & 0.650 & 0.644 & 0.648 & 0.65 & 1.04 & 10 & 13 \\
\hline & ERA5 & 0.574 & 0.582 & 0.578 & 0.578 & 0.571 & 0.570 & 0.58 & 0.79 & 14 & 6 \\
\hline & WFDEI-GPCC & 0.599 & 0.612 & 0.607 & 0.607 & 0.608 & 0.600 & 0.61 & 0.84 & 12 & 8 \\
\hline & WFDEI-CRU & 0.649 & 0.661 & 0.647 & 0.647 & 0.658 & 0.652 & 0.65 & 0.90 & 8 & 11 \\
\hline & \begin{tabular}{|l} 
EWEMBI v1.1 \\
\end{tabular} & 0.599 & 0.612 & 0.607 & 0.607 & 0.608 & 0.600 & 0.61 & 0.84 & 12 & 8 \\
\hline & MERRA-2 & 0.652 & 0.658 & 0.652 & 0.652 & 0.649 & 0.647 & 0.65 & 0.57 & 9 & 3 \\
\hline & JRA-55 & 0.552 & 0.558 & 0.554 & 0.554 & 0.557 & 0.566 & 0.56 & 0.89 & 15 & 10 \\
\hline \multirow{4}{*}{ 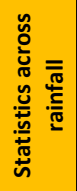 } & Mean & 0.63 & 0.64 & 0.63 & 0.63 & 0.63 & 0.63 & & & & \\
\hline & $V_{2}$ (\%) & 8.8 & 8.7 & 8.4 & 8.4 & 8.9 & 8.7 & & & & \\
\hline & Rank - on mean & 6 & 1 & 5 & 4 & 2 & 3 & & & & \\
\hline & Rank - on $\mathbf{V}_{\mathbf{2}}$ & 5 & 3 & 2 & 1 & 6 & 4 & & & & \\
\hline
\end{tabular}

Table S6. Ranking of rainfall and temperature datasets based on mean Kling-Gupta efficiency $\left(E_{K G}\right)$ of streamflow $(Q)$ over the evaluation period. The mean and the second-order coefficient of variation $\left(V_{2}\right)$ are calculated across 6 temperature datasets for each rainfall dataset, and across 17 rainfall datasets for each temperature dataset. The rank is given from the best (green) to the worst performance (red) for the Volta River basin (VRB)

\begin{tabular}{|c|c|c|c|c|c|c|c|c|c|c|c|}
\hline \multirow{2}{*}{\multicolumn{2}{|c|}{$\begin{array}{c}\text { Kling-Gupta Efficiency of Q } \\
\text { Evaluation period } \\
2008-2012\end{array}$}} & \multicolumn{6}{|c|}{ Temperature datasets } & \multicolumn{4}{|c|}{ Statistics across temperature } \\
\hline & & JRA-55 & MERRA-2 & EWEMBI & WFDEI & ERA5 & PGF v3 & Mean & $V_{2}(\%)$ & $\begin{array}{c}\text { Rank - } \\
\text { on mean }\end{array}$ & $\begin{array}{l}\text { Rank - } \\
\text { on } V_{2}\end{array}$ \\
\hline \multirow{17}{*}{ 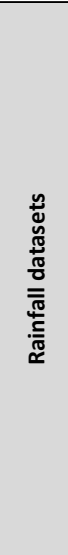 } & TAMSAT v3.0 & 0.674 & 0.726 & 0.676 & 0.676 & 0.667 & 0.683 & 0.68 & 3.11 & 1 & 14 \\
\hline & CHIRPS v2.0 & 0.666 & 0.646 & 0.658 & 0.658 & 0.672 & 0.652 & 0.66 & 1.40 & 3 & 9 \\
\hline & ARC V2.0 & 0.641 & 0.620 & 0.653 & 0.653 & 0.659 & 0.647 & 0.65 & 2.18 & 6 & 13 \\
\hline & RFE v2.0 & 0.575 & 0.537 & 0.645 & 0.645 & 0.629 & 0.597 & 0.60 & 7.13 & 14 & 17 \\
\hline & MSWEP V2.2 & 0.653 & 0.652 & 0.644 & 0.644 & 0.637 & 0.650 & 0.65 & 0.95 & 5 & 6 \\
\hline & GSMaP-std v6 & 0.675 & 0.671 & 0.680 & 0.680 & 0.684 & 0.681 & 0.68 & 0.68 & 2 & 2 \\
\hline & PERSIANN-CDR v1r1 & 0.634 & 0.652 & 0.627 & 0.627 & 0.624 & 0.634 & 0.63 & 1.62 & 11 & 12 \\
\hline & CMORPH-CRT v1.0 & 0.644 & 0.650 & 0.647 & 0.647 & 0.670 & 0.650 & 0.65 & 1.43 & 4 & 10 \\
\hline & TRMM 3B42 v7 & 0.497 & 0.534 & 0.514 & 0.514 & 0.486 & 0.505 & 0.51 & 3.23 & 16 & 15 \\
\hline & TRMM 3B42-RT V7 & 0.529 & 0.542 & 0.532 & 0.532 & 0.525 & 0.531 & 0.53 & 1.05 & 15 & 7 \\
\hline & PGF v3 & 0.633 & 0.646 & 0.635 & 0.635 & 0.635 & 0.634 & 0.64 & 0.74 & 9 & 3 \\
\hline & ERA5 & 0.616 & 0.640 & 0.625 & 0.625 & 0.627 & 0.613 & 0.62 & 1.50 & 13 & 11 \\
\hline & WFDEI-GPCC & 0.636 & 0.647 & 0.645 & 0.645 & 0.635 & 0.635 & 0.64 & 0.87 & 7 & 4 \\
\hline & WFDEI-CRU & 0.634 & 0.631 & 0.640 & 0.640 & 0.639 & 0.633 & 0.64 & 0.64 & 10 & 1 \\
\hline & EWEMBI v1.1 & 0.636 & 0.647 & 0.645 & 0.645 & 0.635 & 0.635 & 0.64 & 0.87 & 7 & 4 \\
\hline & MERRA-2 & 0.629 & 0.634 & 0.630 & 0.630 & 0.615 & 0.634 & 0.63 & 1.12 & 12 & 8 \\
\hline & JRA-55 & 0.343 & 0.383 & 0.347 & 0.347 & 0.351 & 0.355 & 0.35 & 4.05 & 17 & 16 \\
\hline \multirow{4}{*}{ 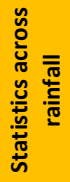 } & Mean & 0.61 & 0.62 & 0.61 & 0.61 & 0.61 & 0.61 & & & & \\
\hline & $V_{2}(\%)$ & 13.6 & 12.5 & 13.1 & 13.1 & 13.6 & 13.0 & & & & \\
\hline & Rank - on mean & 6 & 1 & 3 & 2 & 4 & 5 & & & & \\
\hline & Rank - on $\mathbf{V}_{2}$ & 5 & 1 & 4 & 3 & 6 & 2 & & & & \\
\hline
\end{tabular}


Table S7. Ranking of rainfall and temperature datasets based on mean Pearson correlation coefficient $(r)$ of streamflow $(Q)$ over the calibration period. The mean and the second-order coefficient of variation $\left(V_{2}\right)$ are calculated across 6 temperature datasets for each rainfall dataset, and across 17 rainfall datasets for each temperature dataset. The rank is given from the best (green) to the worst performance (red) for the Volta River basin (VRB)

\begin{tabular}{|c|c|c|c|c|c|c|c|c|c|c|c|}
\hline \multirow{2}{*}{\multicolumn{2}{|c|}{$\begin{array}{c}\text { Correlation of Q } \\
\text { Calibration period } \\
2003-2008\end{array}$}} & \multicolumn{6}{|c|}{ Temperature datasets } & \multicolumn{4}{|c|}{ Statistics across temperature } \\
\hline & & JRA-55 & MERRA-2 & EWEMBI & WFDEI & ERA5 & PGF v3 & Mean & $V_{2}(\%)$ & $\begin{array}{c}\text { Rank - } \\
\text { on mean }\end{array}$ & $\begin{array}{l}\text { Rank - } \\
\text { on } V_{2}\end{array}$ \\
\hline \multirow{17}{*}{ 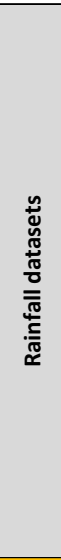 } & TAMSAT v3.0 & 0.904 & 0.900 & 0.902 & 0.902 & 0.892 & 0.903 & 0.90 & 0.46 & 4 & 12 \\
\hline & CHIRPS v2.0 & 0.911 & 0.913 & 0.910 & 0.910 & 0.914 & 0.913 & 0.91 & 0.22 & 1 & 4 \\
\hline & ARC v2.0 & 0.905 & 0.906 & 0.904 & 0.904 & 0.902 & 0.904 & 0.90 & 0.12 & 2 & 1 \\
\hline & RFE v2.0 & 0.898 & 0.895 & 0.901 & 0.901 & 0.901 & 0.896 & 0.90 & 0.33 & 5 & 9 \\
\hline & MSWEP V2.2 & 0.891 & 0.887 & 0.894 & 0.894 & 0.892 & 0.892 & 0.89 & 0.30 & 6 & 7 \\
\hline & GSMaP-std v6 & 0.886 & 0.885 & 0.888 & 0.888 & 0.892 & 0.887 & 0.89 & 0.27 & 10 & 6 \\
\hline & PERSIANN-CDR v1r1 & 0.905 & 0.894 & 0.904 & 0.904 & 0.903 & 0.904 & 0.90 & 0.46 & 3 & 11 \\
\hline & CMORPH-CRT V1.0 & 0.886 & 0.891 & 0.894 & 0.894 & 0.892 & 0.888 & 0.89 & 0.35 & 9 & 10 \\
\hline & TRMM 3B42 v7 & 0.866 & 0.871 & 0.878 & 0.878 & 0.876 & 0.874 & 0.87 & 0.54 & 14 & 16 \\
\hline & TRMM 3B42-RT v7 & 0.869 & 0.874 & 0.875 & 0.875 & 0.873 & 0.873 & 0.87 & 0.26 & 15 & 5 \\
\hline & \begin{tabular}{|l|} 
PGF v3 \\
\end{tabular} & 0.887 & 0.885 & 0.885 & 0.885 & 0.887 & 0.882 & 0.89 & 0.19 & 12 & 3 \\
\hline & ERA5 & 0.864 & 0.859 & 0.866 & 0.866 & 0.855 & 0.863 & 0.86 & 0.52 & 16 & 15 \\
\hline & WFDEI-GPCC & 0.888 & 0.888 & 0.896 & 0.896 & 0.894 & 0.886 & 0.89 & 0.51 & 7 & 13 \\
\hline & WFDEI-CRU & 0.870 & 0.879 & 0.869 & 0.869 & 0.879 & 0.882 & 0.87 & 0.68 & 13 & 17 \\
\hline & EWEMBI v1.1 & 0.888 & 0.888 & 0.896 & 0.896 & 0.894 & 0.886 & 0.89 & 0.51 & 7 & 13 \\
\hline & \begin{tabular}{|l|} 
MERRA-2 \\
\end{tabular} & 0.888 & 0.886 & 0.888 & 0.888 & 0.887 & 0.883 & 0.89 & 0.19 & 11 & 2 \\
\hline & JRA-55 & 0.838 & 0.839 & 0.839 & 0.839 & 0.834 & 0.842 & 0.84 & 0.32 & 17 & 8 \\
\hline \multirow{4}{*}{ 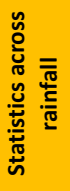 } & Mean & 0.88 & 0.88 & 0.89 & 0.89 & 0.89 & 0.89 & & & & \\
\hline & $V_{2}(\%)$ & 2.1 & 2.0 & 2.0 & 2.0 & 2.2 & 1.9 & & & & \\
\hline & Rank - on mean & 5 & 6 & 2 & 1 & 3 & 4 & & & & \\
\hline & Rank - on $V_{2}$ & 5 & 2 & 3 & 4 & 6 & 1 & & & & \\
\hline
\end{tabular}

Table S8. Ranking of rainfall and temperature datasets based on mean Pearson correlation coefficient $(r)$ of streamflow $(Q)$ over the evaluation period. The mean and the second-order coefficient of variation $\left(V_{2}\right)$ are calculated across 6 temperature datasets for each rainfall dataset, and across 17 rainfall datasets for each temperature dataset. The rank is given from the best (green) to the worst performance (red) for the Volta River basin (VRB)

\begin{tabular}{|c|c|c|c|c|c|c|c|c|c|c|c|}
\hline \multirow{2}{*}{\multicolumn{2}{|c|}{$\begin{array}{l}\text { Correlation of } Q \\
\text { Evaluation period } \\
2008-2012\end{array}$}} & \multicolumn{6}{|c|}{ Temperature datasets } & \multicolumn{4}{|c|}{ Statistics across temperature } \\
\hline & & JRA-55 & MERRA-2 & EWEMBI & WFDEI & ERA5 & PGF v3 & Mean & $V_{2}(\%)$ & $\begin{array}{c}\text { Rank - } \\
\text { on mean }\end{array}$ & $\begin{array}{l}\text { Rank - } \\
\text { on } V_{2}\end{array}$ \\
\hline \multirow{17}{*}{ 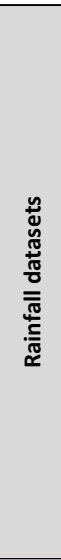 } & TAMSAT v3.0 & 0.896 & 0.900 & 0.895 & 0.895 & 0.891 & 0.896 & 0.90 & 0.31 & 1 & 9 \\
\hline & CHIRPS v2.0 & 0.893 & 0.892 & 0.889 & 0.889 & 0.894 & 0.893 & 0.89 & 0.27 & 2 & 7 \\
\hline & ARC v2.0 & 0.881 & 0.880 & 0.877 & 0.877 & 0.872 & 0.877 & 0.88 & 0.36 & 10 & 11 \\
\hline & RFE v2.0 & 0.880 & 0.876 & 0.884 & 0.884 & 0.881 & 0.879 & 0.88 & 0.34 & 9 & 10 \\
\hline & MSWEP v2.2 & 0.885 & 0.879 & 0.885 & 0.885 & 0.883 & 0.887 & 0.88 & 0.29 & 7 & 8 \\
\hline & GSMaP-std v6 & 0.883 & 0.880 & 0.880 & 0.880 & 0.886 & 0.884 & 0.88 & 0.26 & 8 & 6 \\
\hline & PERSIANN-CDR v1r1 & 0.889 & 0.890 & 0.887 & 0.887 & 0.888 & 0.890 & 0.89 & 0.14 & 3 & 1 \\
\hline & CMORPH-CRT V1.0 & 0.880 & 0.887 & 0.887 & 0.887 & 0.889 & 0.883 & 0.89 & 0.37 & 4 & 12 \\
\hline & TRMM 3B42 v7 & 0.880 & 0.881 & 0.890 & 0.890 & 0.888 & 0.885 & 0.89 & 0.48 & 5 & 14 \\
\hline & TRMM 3B42-RT v7 & 0.872 & 0.876 & 0.877 & 0.877 & 0.873 & 0.875 & 0.87 & 0.24 & 11 & 4 \\
\hline & \begin{tabular}{|l|} 
PGF v3 \\
\end{tabular} & 0.870 & 0.868 & 0.865 & 0.865 & 0.868 & 0.866 & 0.87 & 0.22 & 12 & 3 \\
\hline & ERA5 & 0.800 & 0.811 & 0.800 & 0.800 & 0.807 & 0.794 & 0.80 & 0.73 & 17 & 17 \\
\hline & WFDEI-GPCC & 0.862 & 0.864 & 0.872 & 0.872 & 0.868 & 0.862 & 0.87 & 0.54 & 13 & 15 \\
\hline & WFDEI-CRU & 0.855 & 0.862 & 0.857 & 0.857 & 0.860 & 0.864 & 0.86 & 0.41 & 15 & 13 \\
\hline & EWEMBI v1.1 & 0.862 & 0.864 & 0.872 & 0.872 & 0.868 & 0.862 & 0.87 & 0.54 & 13 & 15 \\
\hline & \begin{tabular}{|l} 
MERRA-2 \\
\end{tabular} & 0.887 & 0.885 & 0.885 & 0.885 & 0.885 & 0.882 & 0.88 & 0.18 & 6 & 2 \\
\hline & \begin{tabular}{|l|} 
JRA-55 \\
\end{tabular} & 0.841 & 0.840 & 0.840 & 0.840 & 0.842 & 0.846 & 0.84 & 0.26 & 16 & 5 \\
\hline \multirow{4}{*}{ 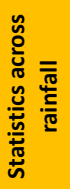 } & Mean & 0.87 & 0.87 & 0.87 & 0.87 & 0.87 & 0.87 & & & & \\
\hline & $V_{2}(\%)$ & 2.7 & 2.4 & 2.6 & 2.6 & 2.5 & 2.8 & & & & \\
\hline & Rank - on mean & 6 & 4 & 2 & 3 & 1 & 5 & & & & \\
\hline & Rank - on V/2 & 5 & 1 & 4 & 3 & 2 & 6 & & & & \\
\hline
\end{tabular}


Table S9. Ranking of rainfall and temperature datasets based on mean bias $(\beta)$ of streamflow $(Q)$ over the calibration period. The mean and the second-order coefficient of variation $\left(V_{2}\right)$ are calculated across 6 temperature datasets for each rainfall dataset, and across 17 rainfall datasets for each temperature dataset. The rank is given from the best (green) to the worst performance (red) for the Volta River basin (VRB)

\begin{tabular}{|c|c|c|c|c|c|c|c|c|c|c|c|}
\hline \multirow{2}{*}{\multicolumn{2}{|c|}{\begin{tabular}{|c|} 
Bias of Q \\
Calibration period \\
2003-2008
\end{tabular}}} & \multicolumn{6}{|c|}{ Temperature datasets } & \multicolumn{4}{|c|}{ Statistics across temperature } \\
\hline & & JRA-55 & MERRA-2 & EWEMBI & WFDEI & ERA5 & PGF v3 & Mean & $V_{2}(\%)$ & $\begin{array}{c}\text { Rank - } \\
\text { on mean }\end{array}$ & $\begin{array}{l}\text { Rank - } \\
\text { on } V_{2}\end{array}$ \\
\hline \multirow{17}{*}{ 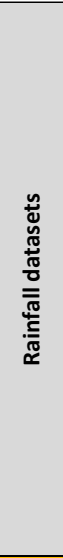 } & TAMSAT v3.0 & 0.869 & 0.895 & 0.869 & 0.869 & 0.906 & 0.878 & 0.88 & 1.77 & 1 & 15 \\
\hline & CHIRPS V2.0 & 0.820 & 0.843 & 0.828 & 0.828 & 0.840 & 0.840 & 0.83 & 1.12 & 10 & 11 \\
\hline & ARC v2.0 & 0.884 & 0.879 & 0.878 & 0.878 & 0.879 & 0.879 & 0.88 & 0.26 & 2 & 1 \\
\hline & RFE v2.0 & 0.872 & 0.881 & 0.871 & 0.871 & 0.870 & 0.869 & 0.87 & 0.49 & 5 & 3 \\
\hline & MSWEP v2.2 & 0.831 & 0.821 & 0.829 & 0.829 & 0.835 & 0.832 & 0.83 & 0.57 & 11 & 5 \\
\hline & GSMaP-std v6 & 0.881 & 0.885 & 0.870 & 0.870 & 0.887 & 0.876 & 0.88 & 0.81 & 3 & 8 \\
\hline & PERSIANN-CDR v1r1 & 0.859 & 0.857 & 0.856 & 0.856 & 0.855 & 0.864 & 0.86 & 0.38 & 6 & 2 \\
\hline & CMORPH-CRT v1.0 & 0.874 & 0.881 & 0.886 & 0.886 & 0.868 & 0.872 & 0.88 & 0.85 & 4 & 9 \\
\hline & TRMM 3B42 v7 & 0.747 & 0.755 & 0.781 & 0.782 & 0.741 & 0.768 & 0.76 & 2.27 & 16 & 17 \\
\hline & TRMM 3B42-RT v7 & 0.815 & 0.794 & 0.824 & 0.824 & 0.791 & 0.822 & 0.81 & 1.87 & 12 & 16 \\
\hline & PGF v3 & 0.832 & 0.845 & 0.846 & 0.846 & 0.820 & 0.846 & 0.84 & 1.29 & 8 & 12 \\
\hline & ERA5 & 0.764 & 0.762 & 0.779 & 0.779 & 0.755 & 0.762 & 0.77 & 1.30 & 15 & 13 \\
\hline & WFDEI-GPCC & 0.795 & 0.804 & 0.807 & 0.807 & 0.801 & 0.792 & 0.80 & 0.76 & 13 & 6 \\
\hline & WFDEI-CRU & 0.850 & 0.852 & 0.852 & 0.852 & 0.841 & 0.853 & 0.85 & 0.53 & 7 & 4 \\
\hline & EWEMBI v1.1 & 0.795 & 0.804 & 0.807 & 0.807 & 0.801 & 0.792 & 0.80 & 0.76 & 13 & 6 \\
\hline & MERRA-2 & 0.837 & 0.839 & 0.831 & 0.831 & 0.855 & 0.823 & 0.84 & 1.33 & 9 & 14 \\
\hline & \begin{tabular}{|l|}
$J R A-55$ \\
\end{tabular} & 0.751 & 0.760 & 0.762 & 0.762 & 0.750 & 0.772 & 0.76 & 1.09 & 17 & 10 \\
\hline \multirow{4}{*}{ 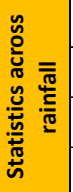 } & Mean & 0.83 & 0.83 & 0.83 & 0.83 & 0.83 & 0.83 & & & & \\
\hline & $V_{2}(\%)$ & 5.4 & 5.5 & 4.5 & 4.4 & 6.0 & 4.9 & & & & \\
\hline & Rank - on mean & 6 & 3 & 2 & 1 & 5 & 4 & & & & \\
\hline & Rank - on $V_{2}$ & 4 & 5 & 2 & 1 & 6 & 3 & & & & \\
\hline
\end{tabular}

Table S10. Ranking of rainfall and temperature datasets based on mean bias $(\beta)$ of streamflow $(Q)$ over the evaluation period. The mean and the second-order coefficient of variation $\left(V_{2}\right)$ are calculated across 6 temperature datasets for each rainfall dataset, and across 17 rainfall datasets for each temperature dataset. The rank is given from the best (green) to the worst performance (red) for the Volta River basin (VRB)

\begin{tabular}{|c|c|c|c|c|c|c|c|c|c|c|c|}
\hline \multirow{2}{*}{\multicolumn{2}{|c|}{$\begin{array}{c}\text { Bias of Q } \\
\text { Evaluation period } \\
\text { 2008-2012 }\end{array}$}} & \multicolumn{6}{|c|}{ Temperature datasets } & \multicolumn{4}{|c|}{ Statistics across temperature } \\
\hline & & JRA-55 & MERRA-2 & EWEMBI & WFDEI & ERA5 & PGF v3 & Mean & $V_{2}(\%)$ & $\begin{array}{c}\text { Rank - } \\
\text { on mean }\end{array}$ & $\begin{array}{l}\text { Rank - } \\
\text { on } V_{2}\end{array}$ \\
\hline \multirow{17}{*}{ 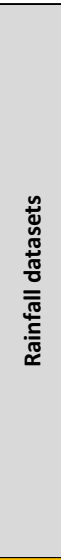 } & TAMSAT v3.0 & 0.788 & 0.900 & 0.779 & 0.779 & 0.797 & 0.798 & 0.81 & 5.74 & 10 & 15 \\
\hline & CHIRPS v2.0 & 0.896 & 0.908 & 0.918 & 0.918 & 0.886 & 0.871 & 0.90 & 2.06 & 5 & 2 \\
\hline & ARC v2.0 & 1.054 & 1.105 & 1.020 & 1.020 & 0.995 & 1.040 & 1.04 & 3.66 & 1 & 8 \\
\hline & RFE v2.0 & 1.161 & 1.217 & 1.006 & 1.006 & 1.056 & 1.118 & 1.09 & 7.85 & 3 & 16 \\
\hline & MSWEP V2.2 & 0.754 & 0.774 & 0.740 & 0.740 & 0.727 & 0.749 & 0.75 & 2.14 & 14 & 3 \\
\hline & GSMaP-std v6 & 0.903 & 0.940 & 0.879 & 0.879 & 0.863 & 0.884 & 0.89 & 3.04 & 7 & 6 \\
\hline & PERSIANN-CDR v1r1 & 0.749 & 0.794 & 0.730 & 0.730 & 0.710 & 0.746 & 0.74 & 3.83 & 15 & 9 \\
\hline & CMORPH-CRT V1.0 & 0.961 & 0.987 & 0.907 & 0.907 & 0.862 & 0.951 & 0.93 & 4.88 & 2 & 14 \\
\hline & TRMM 3B42 v7 & 0.580 & 0.615 & 0.611 & 0.611 & 0.557 & 0.602 & 0.60 & 3.87 & 16 & 10 \\
\hline & TRMM 3B42-RT v7 & 0.801 & 0.852 & 0.782 & 0.782 & 0.754 & 0.787 & 0.79 & 4.12 & 11 & 12 \\
\hline & PGF v3 & 0.841 & 0.897 & 0.834 & 0.834 & 0.791 & 0.845 & 0.84 & 4.02 & 9 & 11 \\
\hline & ERA5 & 0.984 & 0.827 & 0.938 & 0.938 & 0.779 & 0.966 & 0.91 & 9.10 & 4 & 17 \\
\hline & WFDEI-GPCC & 0.781 & 0.816 & 0.777 & 0.777 & 0.752 & 0.769 & 0.78 & 2.69 & 12 & 4 \\
\hline & WFDEI-CRU & 0.909 & 0.947 & 0.887 & 0.887 & 0.865 & 0.891 & 0.90 & 3.14 & 6 & 7 \\
\hline & EWEMBI v1.1 & 0.781 & 0.816 & 0.777 & 0.777 & 0.752 & 0.769 & 0.78 & 2.69 & 12 & 4 \\
\hline & \begin{tabular}{|l|} 
MERRA-2 \\
\end{tabular} & 0.896 & 0.915 & 0.871 & 0.871 & 0.875 & 0.882 & 0.89 & 1.94 & 8 & 1 \\
\hline & JRA-55 & 0.409 & 0.458 & 0.419 & 0.419 & 0.415 & 0.415 & 0.42 & 4.18 & 17 & 13 \\
\hline \multirow{4}{*}{ 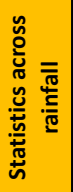 } & Mean & 0.84 & 0.87 & 0.82 & 0.82 & 0.79 & 0.83 & & & & \\
\hline & $V_{2}(\%)$ & 20.2 & 19.2 & 17.6 & 17.6 & 18.5 & 19.2 & & & & \\
\hline & Rank - on mean & 2 & 1 & 5 & 4 & 6 & 3 & & & & \\
\hline & Rank - on $V_{2}$ & 6 & 5 & 2 & 1 & 3 & 4 & & & & \\
\hline
\end{tabular}


Table S11. Ranking of rainfall and temperature datasets based on mean variability $(\gamma)$ of streamflow $(Q)$ over the calibration period. The mean and the second-order coefficient of variation $\left(V_{2}\right)$ are calculated across 6 temperature datasets for each rainfall dataset, and across 17 rainfall datasets for each temperature dataset. The rank is given from the best (green) to the worst performance (red) for the Volta River basin (VRB)

\begin{tabular}{|c|c|c|c|c|c|c|c|c|c|c|c|}
\hline \multirow{2}{*}{\multicolumn{2}{|c|}{$\begin{array}{c}\text { Variability of Q } \\
\text { Calibration period } \\
2003-2008\end{array}$}} & \multicolumn{6}{|c|}{ Temperature datasets } & \multicolumn{4}{|c|}{ Statistics across temperature } \\
\hline & & JRA-55 & MERRA-2 & EWEMBI & WFDEI & ERA5 & PGF v3 & Mean & $V_{2}(\%)$ & $\begin{array}{c}\text { Rank - } \\
\text { on mean }\end{array}$ & $\begin{array}{l}\text { Rank - } \\
\text { on } V_{2}\end{array}$ \\
\hline \multirow{17}{*}{ 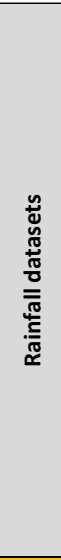 } & TAMSAT v3.0 & 0.969 & 0.995 & 0.987 & 0.987 & 0.918 & 0.983 & 0.97 & 2.91 & 6 & 16 \\
\hline & CHIRPS V2.0 & 0.992 & 0.988 & 0.991 & 0.991 & 0.944 & 0.995 & 0.98 & 1.96 & 3 & 13 \\
\hline & ARC v2.0 & 0.950 & 0.968 & 0.971 & 0.971 & 0.983 & 0.971 & 0.97 & 1.12 & 10 & 4 \\
\hline & RFE v2.0 & 0.969 & 0.984 & 0.970 & 0.970 & 0.968 & 0.994 & 0.98 & 1.07 & 4 & 2 \\
\hline & MSWEP V2.2 & 0.935 & 0.954 & 0.924 & 0.924 & 0.924 & 0.932 & 0.93 & 1.26 & 15 & 6 \\
\hline & GSMaP-std v6 & 0.927 & 0.957 & 0.967 & 0.967 & 0.932 & 0.954 & 0.95 & 1.79 & 12 & 10 \\
\hline & PERSIANN-CDR v1r1 & 0.983 & 0.943 & 0.987 & 0.987 & 0.965 & 0.972 & 0.97 & 1.77 & 7 & 9 \\
\hline & CMORPH-CRT V1.0 & 0.964 & 0.940 & 0.915 & 0.915 & 0.951 & 0.951 & 0.94 & 2.17 & 13 & 14 \\
\hline & TRMM 3B42 v7 & 0.893 & 0.898 & 0.849 & 0.849 & 0.889 & 0.853 & 0.87 & 2.74 & 17 & 15 \\
\hline & TRMM 3B42-RT v7 & 0.920 & 0.917 & 0.901 & 0.901 & 0.920 & 0.898 & 0.91 & 1.15 & 16 & 5 \\
\hline & PGF v3 & 0.960 & 0.977 & 0.970 & 0.970 & 0.991 & 0.983 & 0.98 & 1.11 & 5 & 3 \\
\hline & ERA5 & 0.953 & 0.990 & 0.924 & 0.924 & 0.986 & 0.945 & 0.95 & 3.02 & 11 & 17 \\
\hline & WFDEI-GPCC & 0.988 & 0.984 & 0.948 & 0.948 & 0.972 & 0.983 & 0.97 & 1.90 & 8 & 11 \\
\hline & \begin{tabular}{|l|} 
WFDEI-CRU \\
\end{tabular} & 0.995 & 0.982 & 0.988 & 0.988 & 0.997 & 0.955 & 0.98 & 1.55 & 2 & 7 \\
\hline & EWEMBI v1.1 & 0.988 & 0.984 & 0.948 & 0.948 & 0.972 & 0.983 & 0.97 & 1.90 & 8 & 11 \\
\hline & MERRA-2 & 0.980 & 0.977 & 0.987 & 0.987 & 0.986 & 0.994 & 0.99 & 0.61 & 1 & 1 \\
\hline & JRA-55 & 0.937 & 0.922 & 0.917 & 0.917 & 0.942 & 0.958 & 0.93 & 1.77 & 14 & 8 \\
\hline \multirow{4}{*}{ 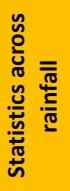 } & Mean & 0.96 & 0.96 & 0.95 & 0.95 & 0.96 & 0.96 & & & & \\
\hline & $V_{2}$ (\%) & 3.0 & 3.0 & 4.2 & 4.2 & 3.2 & 3.9 & & & & \\
\hline & Rank - on mean & 2 & 1 & 5 & 6 & 4 & 3 & & & & \\
\hline & Rank - on $V_{2}$ & 1 & 2 & 6 & 5 & 3 & 4 & & & & \\
\hline
\end{tabular}

Table S12. Ranking of rainfall and temperature datasets based on mean variability $(\gamma)$ of streamflow $(Q)$ over the evaluation period. The mean and the second-order coefficient of variation $\left(V_{2}\right)$ are calculated across 6 temperature datasets for each rainfall dataset, and across 17 rainfall datasets for each temperature dataset. The rank is given from the best (green) to the worst performance (red) for the Volta River basin (VRB)

\begin{tabular}{|c|c|c|c|c|c|c|c|c|c|c|c|}
\hline \multirow{2}{*}{\multicolumn{2}{|c|}{$\begin{array}{c}\text { Variability of Q } \\
\text { Evaluation period } \\
2008-2012\end{array}$}} & \multicolumn{6}{|c|}{ Temperature datasets } & \multicolumn{4}{|c|}{ Statistics across temperature } \\
\hline & & JRA-55 & MERRA-2 & EWEMBI & WFDEI & ERA5 & PGF v3 & Mean & $V_{2}(\%)$ & $\begin{array}{c}\text { Rank - } \\
\text { on mean }\end{array}$ & $\begin{array}{l}\text { Rank - } \\
\text { on } V_{2}\end{array}$ \\
\hline \multirow{17}{*}{ 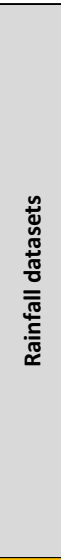 } & TAMSAT v3.0 & 0.896 & 0.920 & 0.912 & 0.912 & 0.859 & 0.907 & 0.90 & 2.46 & 14 & 16 \\
\hline & CHIRPS v2.0 & 0.985 & 0.993 & 0.986 & 0.986 & 0.954 & 0.990 & 0.98 & 1.45 & 4 & 8 \\
\hline & ARC v2.0 & 0.970 & 0.975 & 0.975 & 0.975 & 1.006 & 0.979 & 0.98 & 1.32 & 5 & 5 \\
\hline & RFE v2.0 & 0.940 & 0.940 & 0.935 & 0.935 & 0.952 & 0.952 & 0.94 & 0.86 & 10 & 2 \\
\hline & MSWEP V2.2 & 0.943 & 0.958 & 0.928 & 0.928 & 0.943 & 0.939 & 0.94 & 1.21 & 12 & 3 \\
\hline & GSMaP-std v6 & 0.949 & 0.973 & 0.979 & 0.979 & 0.961 & 0.969 & 0.97 & 1.22 & 7 & 4 \\
\hline & PERSIANN-CDR v1r1 & 0.970 & 0.917 & 0.974 & 0.974 & 0.958 & 0.957 & 0.96 & 2.24 & 9 & 14 \\
\hline & CMORPH-CRT V1.0 & 0.942 & 0.922 & 0.892 & 0.892 & 0.936 & 0.929 & 0.92 & 2.38 & 13 & 15 \\
\hline & TRMM 3B42 v7 & 0.822 & 0.848 & 0.787 & 0.787 & 0.821 & 0.788 & 0.81 & 3.17 & 16 & 17 \\
\hline & TRMM 3B42-RT v7 & 0.838 & 0.833 & 0.814 & 0.814 & 0.838 & 0.816 & 0.83 & 1.46 & 15 & 9 \\
\hline & PGF v3 & 0.971 & 0.980 & 0.964 & 0.964 & 0.998 & 0.968 & 0.97 & 1.34 & 6 & 6 \\
\hline & ERA5 & 0.964 & 0.986 & 0.941 & 0.941 & 0.987 & 0.968 & 0.96 & 2.11 & 8 & 13 \\
\hline & WFDEI-GPCC & 1.008 & 1.002 & 0.966 & 0.966 & 0.998 & 0.998 & 0.99 & 1.87 & 2 & 10 \\
\hline & WFDEI-CRU & 1.004 & 1.010 & 0.999 & 0.999 & 1.026 & 0.985 & 1.00 & 1.37 & 1 & 7 \\
\hline & EWEMBI v1.1 & 1.008 & 1.002 & 0.966 & 0.966 & 0.998 & 0.998 & 0.99 & 1.87 & 2 & 10 \\
\hline & MERRA-2 & 0.935 & 0.937 & 0.939 & 0.939 & 0.957 & 0.943 & 0.94 & 0.83 & 11 & 1 \\
\hline & JRA-55 & 0.796 & 0.783 & 0.779 & 0.779 & 0.804 & 0.819 & 0.79 & 2.01 & 17 & 12 \\
\hline \multirow{4}{*}{ 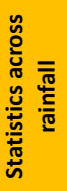 } & Mean & 0.94 & 0.94 & 0.93 & 0.93 & 0.94 & 0.94 & & & & \\
\hline & $V_{2}(\%)$ & 6.8 & 6.9 & 7.4 & 7.4 & 7.3 & 7.0 & & & & \\
\hline & Rank - on mean & 3 & 2 & 6 & 5 & 1 & 4 & & & & \\
\hline & Rank - on $V_{2}$ & 1 & 2 & 6 & 5 & 4 & 3 & & & & \\
\hline
\end{tabular}




\subsection{Boxplots and range plots}

\subsubsection{Combined calibration and evaluation periods}
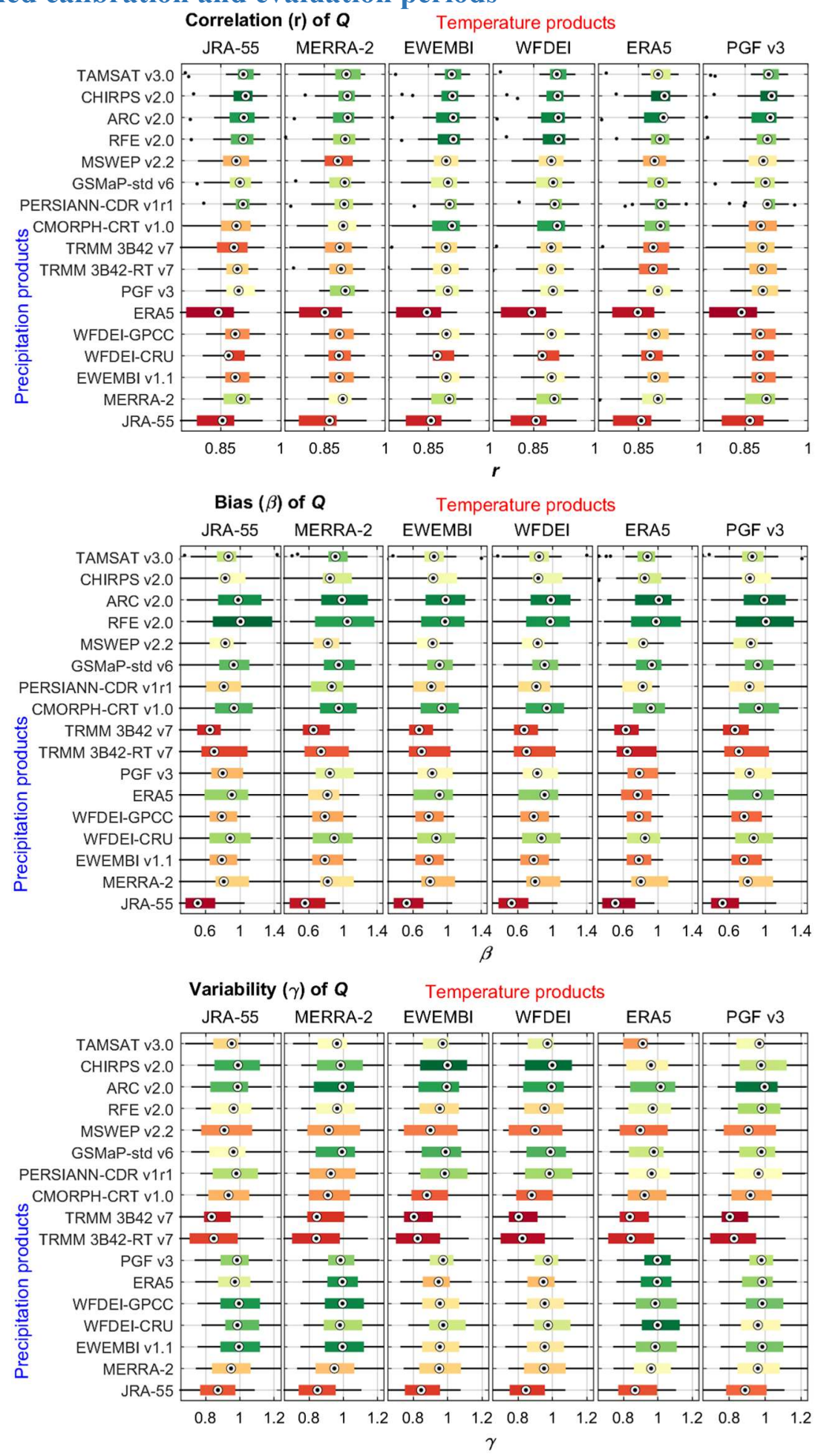

Figure S8. Pearson correlation coefficient $(r)$, bias $(\beta)$ and variability $(\gamma)$ of daily streamflow (Q) over the simulation period (2003-2012) using different combinations of precipitation and temperature datasets as inputs for hydrological modelling. Each boxplot has 22 values representing the combined performance of the calibration and evaluation periods for 11 streamflow stations. The boxplots are colored from the best (green) to the worst performance (red) based on the median value. 


\subsubsection{Separated calibration and evaluation periods}
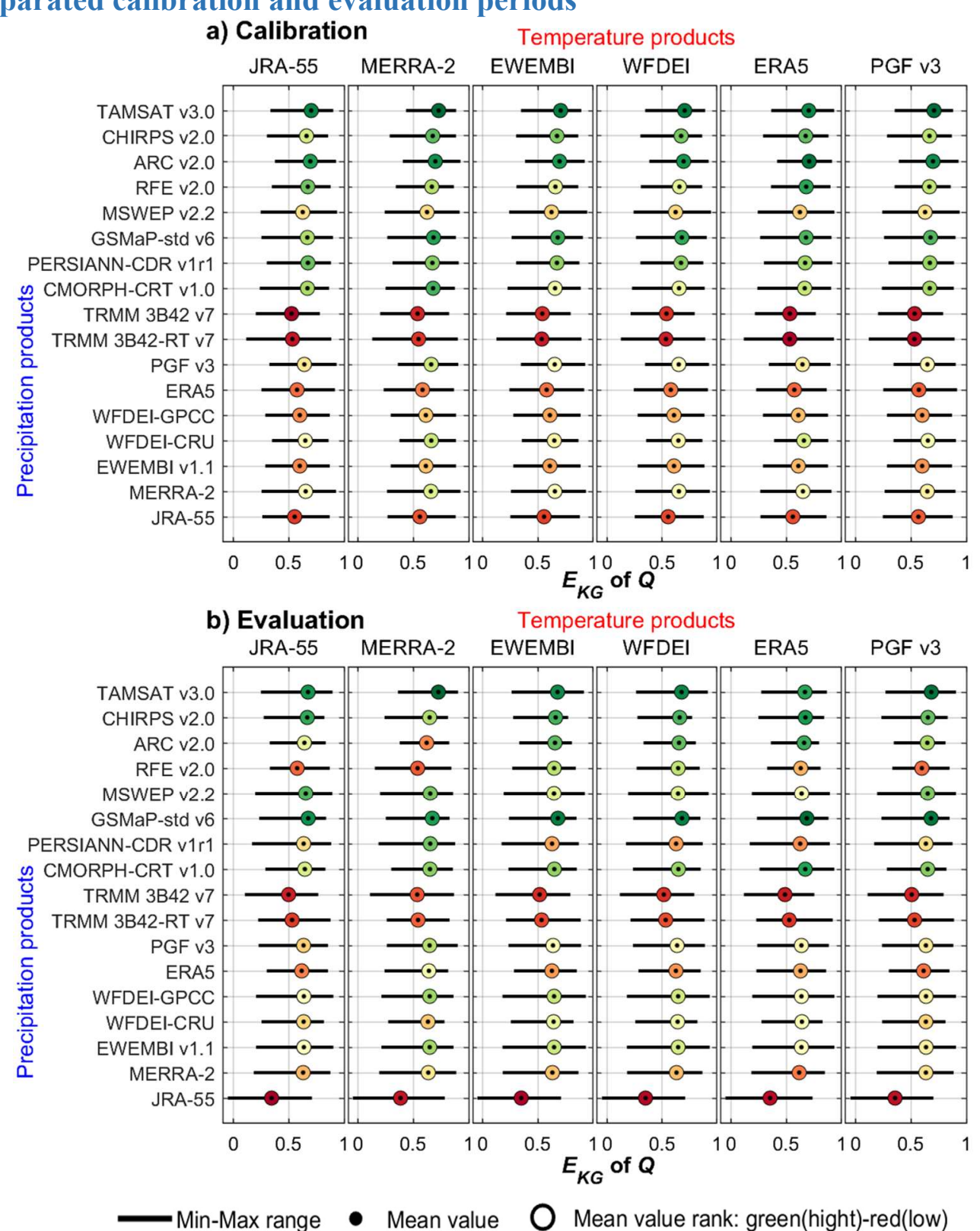

Figure S9. Kling-Gupta efficiency $\left(E_{K G}\right)$ of daily streamflow $(Q)$ over (a) the calibration (2003-2008) and (b) the evaluation (2009-2012) periods for the Volta River basin (VRB), using different combinations of precipitation and temperature datasets as inputs for hydrological modelling. The dots give the mean score and the bars represent the min-max range of values for 11 streamflow gauges. The mean values are colored from the best (green) to the worst performance (red). 
a) Calibration

Temperature products

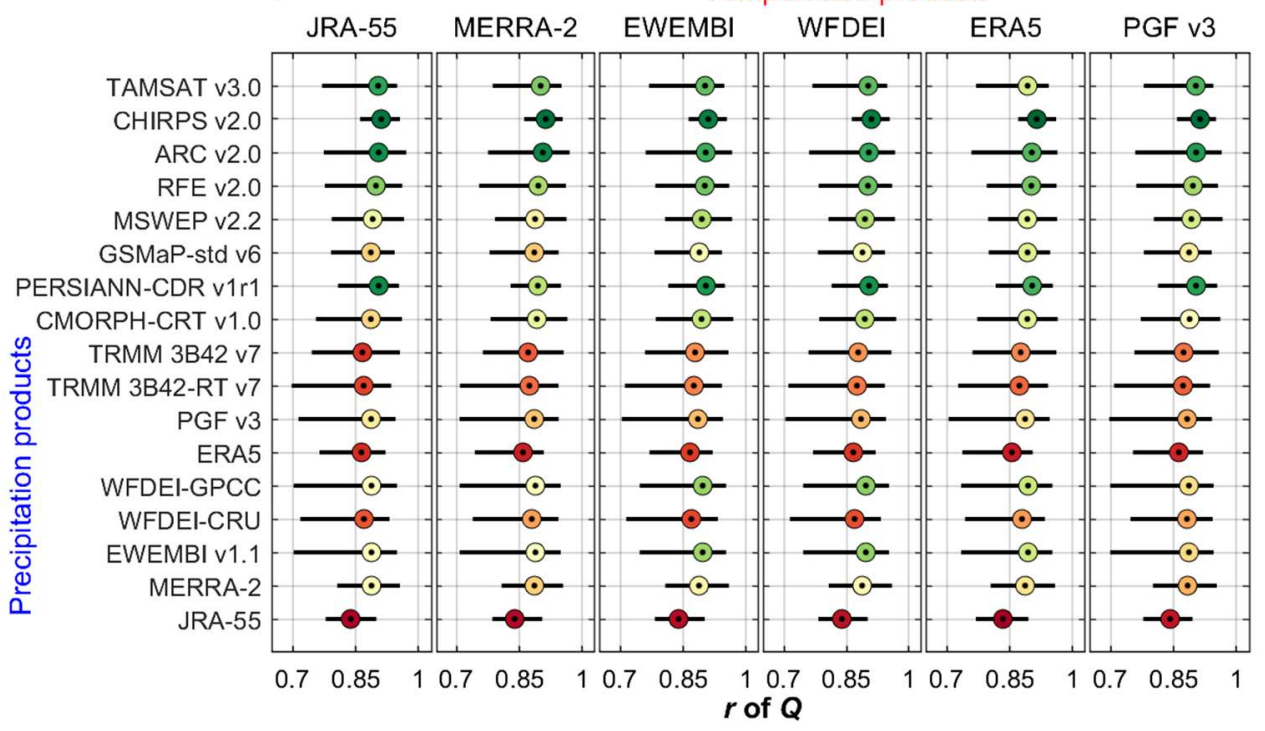

b) Evaluation

Temperature products

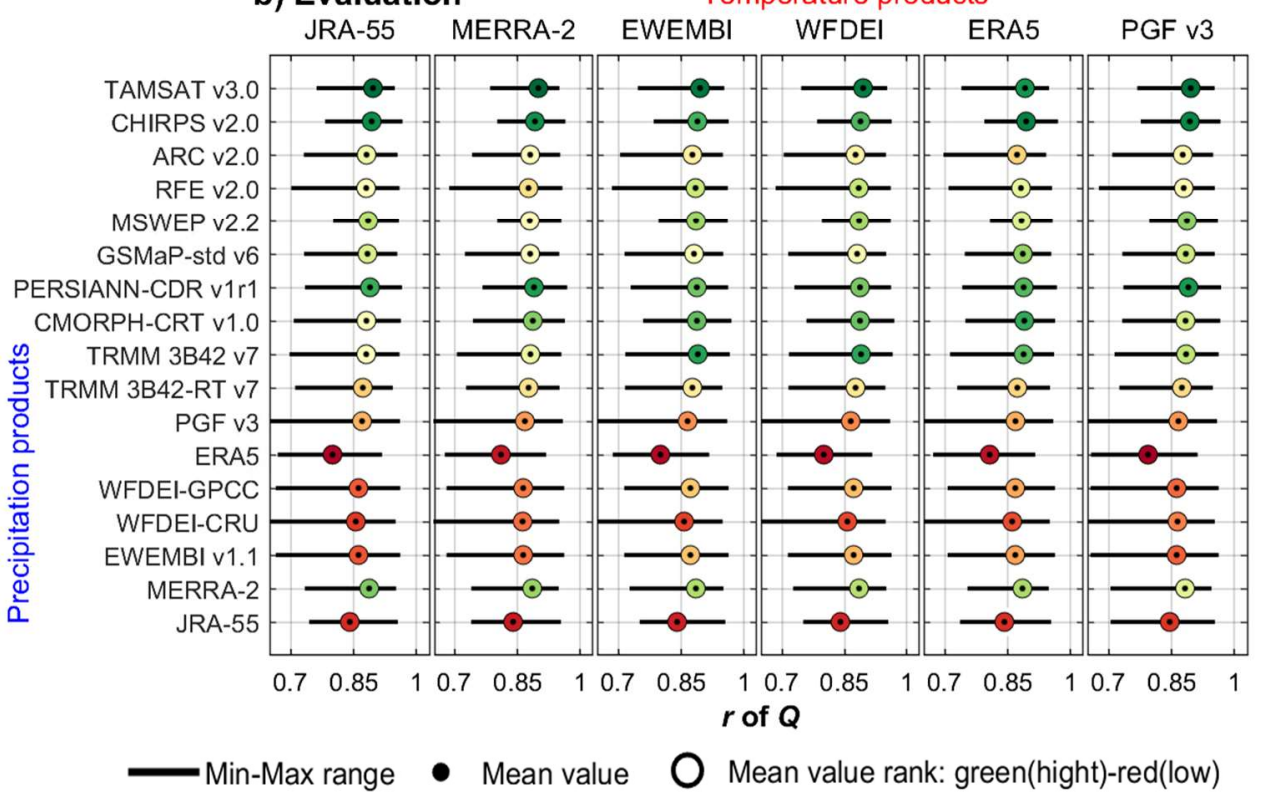

Figure S10. Pearson correlation coefficient $(r)$ of daily streamflow $(Q)$ over (a) the calibration (2003-2008) and (b) the evaluation (2009-2012) periods for the Volta River basin (VRB), using different combinations of precipitation and temperature datasets as inputs for hydrological modelling. The dots give the mean score and the bars represent the min-max range of values for 11 streamflow gauges. The mean values are colored from the best (green) to the worst performance (red). 
a) Calibration

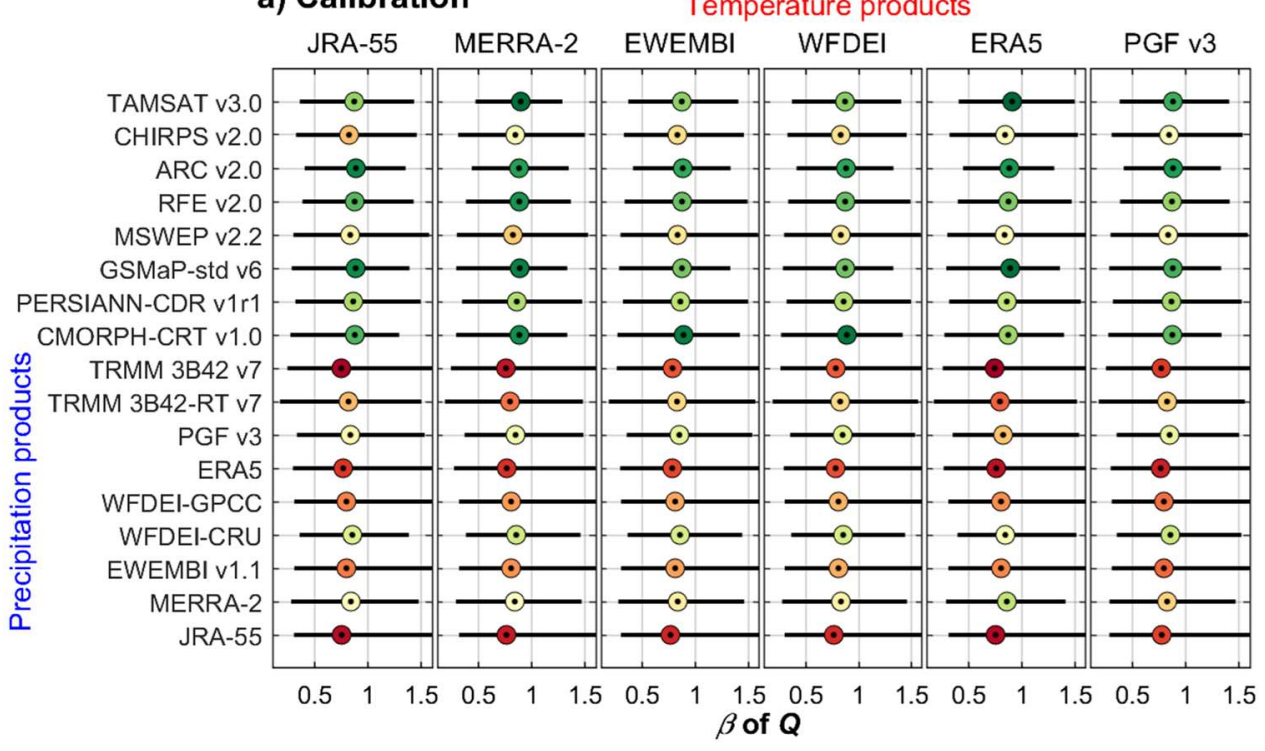

b) Evaluation

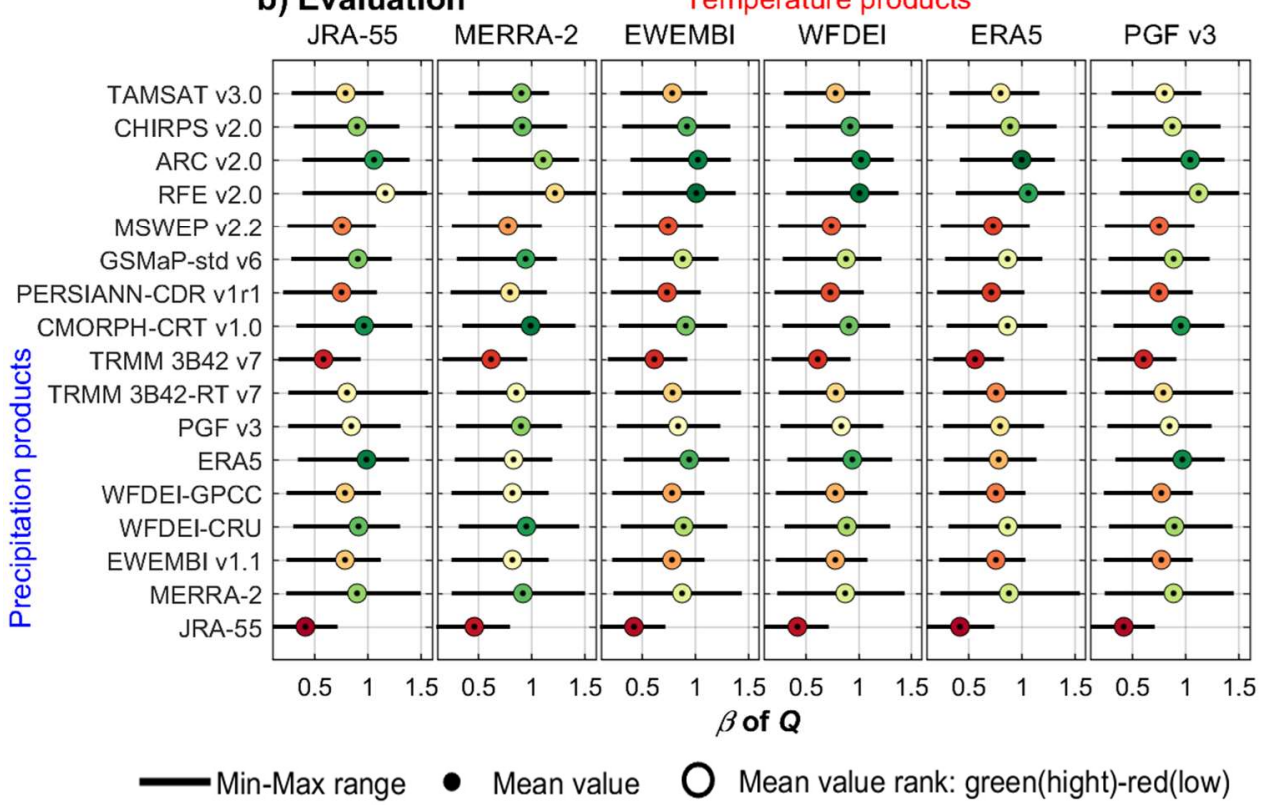

Figure S11. Bias $(\beta)$ of daily streamflow $(Q)$ over (a) the calibration (2003-2008) and (b) the evaluation (20092012) periods for the Volta River basin (VRB), using different combinations of precipitation and temperature datasets as inputs for hydrological modelling. The dots give the mean score and the bars represent the min-max range of values for 11 streamflow gauges. The mean values are colored from the best (green) to the worst performance (red). 

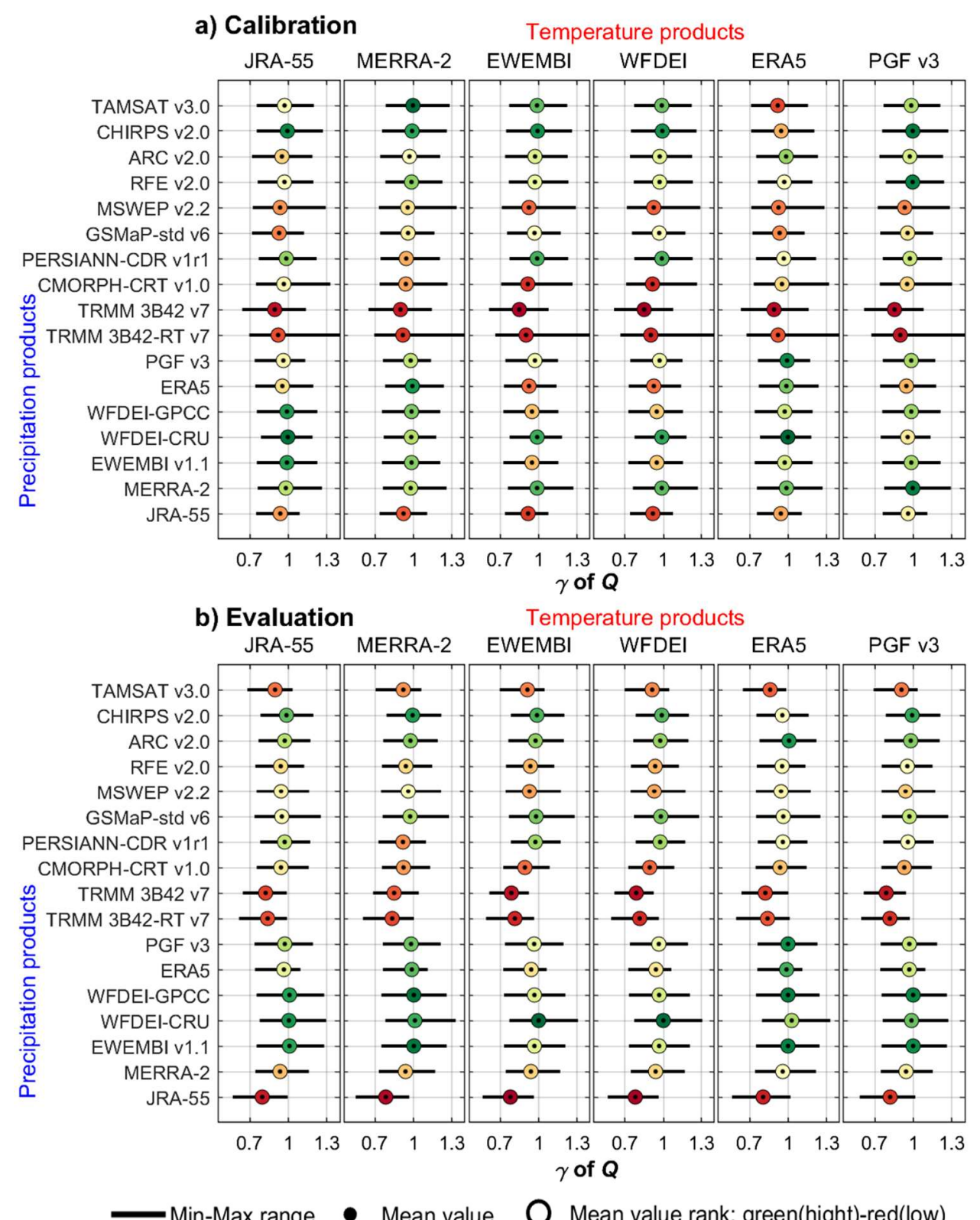

Figure S12. Variability $(\gamma)$ of daily streamflow $(Q)$ over (a) the calibration (2003-2008) and (b) the evaluation (2009-2012) periods for the Volta River basin (VRB), using different combinations of precipitation and temperature datasets as inputs for hydrological modelling. The dots give the mean score and the bars represent the min-max range of values for 11 streamflow gauges. The mean values are colored from the best (green) to the worst performance (red). 


\section{Model performance for terrestrial water storage \\ 6.1 Ranking of rainfall and temperature datasets}

Table S13. Ranking of rainfall and temperature datasets based on the Pearson correlation coefficient $(r)$ of terrestrial water storage $(S t)$ over the simulation period. The mean and the second-order coefficient of variation $\left(V_{2}\right)$ are calculated across 6 temperature datasets for each rainfall dataset, and across 17 rainfall datasets for each temperature dataset. The rank is given from the best (green) to the worst performance (red) for four climatic zones in the Volta River basin (VRB)

\begin{tabular}{|c|c|c|c|c|c|c|c|c|c|c|c|c|c|c|c|c|c|c|c|c|c|}
\hline \multirow{2}{*}{\multicolumn{2}{|c|}{$\begin{array}{l}\text { Pearson's correlation } \\
\text { coefficient of St } \\
\text { Simulation period } \\
2003-2012\end{array}$}} & \multicolumn{5}{|c|}{ Mean } & \multicolumn{5}{|c|}{ V2 (\%) } & \multicolumn{5}{|c|}{ Rank - on mean } & \multicolumn{5}{|c|}{ Rank - on V2 } \\
\hline & & 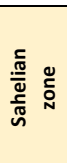 & 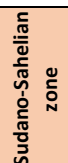 & 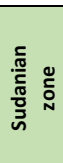 & 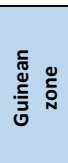 & $\begin{array}{l}\mathscr{\mathscr { q }} \\
\stackrel{\sim}{>}\end{array}$ & 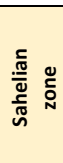 & 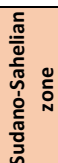 & 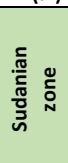 & 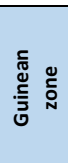 & 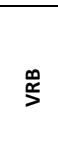 & 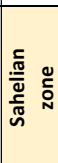 & 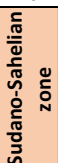 & 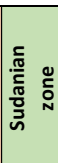 & 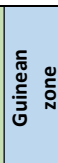 & $\stackrel{\mathscr{m}}{\stackrel{\sim}{>}}$ & 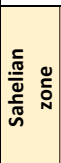 & 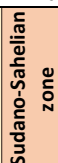 & 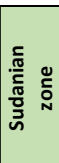 & 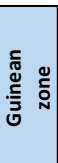 & $\stackrel{\infty}{\stackrel{\infty}{>}}$ \\
\hline \multirow{17}{*}{ 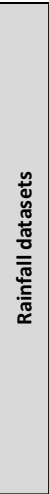 } & TAMSAT v3.0 & 0.686 & 0.777 & 0.857 & 0.740 & 0.856 & 5.01 & 2.90 & 3.92 & 6.39 & 3.37 & 9 & 3 & 3 & 2 & 6 & 12 & 8 & 10 & 11 & 8 \\
\hline & CHIRPS v2.0 & 0.671 & 0.740 & 0.824 & 0.715 & 0.863 & 7.38 & 2.14 & 2.60 & 3.55 & 1.49 & 12 & 7 & 8 & 5 & 4 & 14 & 4 & 5 & 6 & 3 \\
\hline & ARC v2.0 & 0.732 & 0.799 & 0.918 & 0.667 & 0.910 & 1.74 & 2.14 & 1.34 & 1.85 & 1.30 & 1 & 1 & 1 & 8 & 1 & 2 & 3 & 3 & 1 & 1 \\
\hline & RFE v2.0 & 0.709 & 0.780 & 0.890 & 0.648 & 0.882 & 3.88 & 3.14 & 4.66 & 3.82 & 4.56 & 4 & 2 & 2 & 10 & 2 & 8 & 9 & 11 & 7 & 11 \\
\hline & MSWEP v2.2 & 0.579 & 0.681 & 0.728 & 0.490 & 0.719 & 3.07 & 2.80 & 3.63 & 6.71 & 3.54 & 15 & 14 & 16 & 16 & 16 & 6 & 6 & 9 & 12 & 9 \\
\hline & GSMaP-std v6 & 0.718 & 0.756 & 0.845 & 0.656 & 0.842 & 3.29 & 2.65 & 1.33 & 3.33 & 1.63 & 3 & 5 & 5 & 9 & 7 & 7 & 5 & 2 & 4 & 4 \\
\hline & PERSIANN-CDR v1r1 & 0.594 & 0.718 & 0.807 & 0.637 & 0.804 & 3.91 & 3.20 & 2.94 & 7.68 & 2.70 & 14 & 10 & 11 & 11 & 11 & 9 & 10 & 7 & 13 & 7 \\
\hline & CMORPH-CRT V1.0 & 0.691 & 0.764 & 0.856 & 0.775 & 0.875 & 4.06 & 4.31 & 5.46 & 4.09 & 5.16 & 7 & 4 & 4 & 1 & 3 & 10 & 11 & 13 & 9 & 13 \\
\hline & TRMM 3B42 v7 & 0.671 & 0.717 & 0.780 & 0.516 & 0.769 & 5.74 & 5.96 & 6.72 & 8.29 & 5.93 & 11 & 11 & 13 & 15 & 13 & 13 & 13 & 14 & 16 & 14 \\
\hline & TRMM 3B42-RT v7 & 0.677 & 0.744 & 0.834 & 0.724 & 0.860 & 1.37 & 1.63 & 0.79 & 2.04 & 1.38 & 10 & 6 & 6 & 3 & 5 & 1 & 1 & 1 & 2 & 2 \\
\hline & \begin{tabular}{|l|} 
PGF v3 \\
\end{tabular} & 0.688 & 0.729 & 0.824 & 0.715 & 0.823 & 4.50 & 2.85 & 2.63 & 3.47 & 2.44 & 8 & 9 & 9 & 4 & 9 & 11 & 7 & 6 & 5 & 6 \\
\hline & ERA5 & 0.574 & 0.704 & 0.814 & 0.691 & 0.823 & 11.55 & 7.68 & 3.12 & 2.08 & 4.18 & 16 & 13 & 10 & 7 & 10 & 17 & 15 & 8 & 3 & 10 \\
\hline & WFDEI-GPCC & 0.707 & 0.678 & 0.756 & 0.546 & 0.748 & 8.67 & 8.21 & 8.29 & 7.98 & 7.48 & 5 & 15 & 14 & 13 & 14 & 15 & 16 & 15 & 14 & 15 \\
\hline & WFDEI-CRU & 0.720 & 0.730 & 0.829 & 0.697 & 0.829 & 2.99 & 4.39 & 5.23 & 5.17 & 4.64 & 2 & 8 & 7 & 6 & 8 & 5 & 12 & 12 & 10 & 12 \\
\hline & EWEMBI v1.1 & 0.707 & 0.678 & 0.756 & 0.546 & 0.748 & 8.67 & 8.21 & 8.29 & 7.98 & 7.48 & 5 & 15 & 14 & 13 & 14 & 15 & 16 & 15 & 14 & 15 \\
\hline & \begin{tabular}{|l|} 
MERRA-2 \\
\end{tabular} & 0.558 & 0.712 & 0.807 & 0.615 & 0.800 & 2.75 & 1.85 & 2.34 & 4.09 & 2.10 & 17 & 12 & 12 & 12 & 12 & 4 & 2 & 4 & 8 & 5 \\
\hline & JRA-55 & 0.658 & 0.558 & 0.385 & -0.185 & 0.377 & 2.55 & 7.48 & 30.03 & 25.54 & 20.23 & 13 & 17 & 17 & 17 & 17 & 3 & 14 & 17 & 17 & 17 \\
\hline \multirow{6}{*}{ 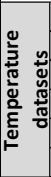 } & JRA-55 & 0.682 & 0.733 & 0.817 & 0.609 & 0.811 & 9.85 & 6.47 & 8.86 & 31.60 & 11.08 & 1 & 2 & 1 & 2 & 1 & 4 & 1 & 1 & 1 & 1 \\
\hline & MERRA-2 & 0.659 & 0.706 & 0.785 & 0.594 & 0.785 & 10.88 & 9.00 & 15.83 & 36.09 & 16.01 & 6 & 6 & 4 & 3 & 4 & 5 & 3 & 3 & 5 & 3 \\
\hline & EWEMBI & 0.663 & 0.713 & 0.776 & 0.593 & 0.781 & 8.52 & 9.52 & 16.98 & 34.65 & 16.68 & 4 & 4 & 6 & 5 & 6 & 3 & 6 & 6 & 4 & 6 \\
\hline & $\sqrt{W F D E I}$ & 0.663 & 0.713 & 0.776 & 0.593 & 0.781 & 8.52 & 9.52 & 16.83 & 34.60 & 16.63 & 5 & 5 & 5 & 4 & 5 & 2 & 5 & 5 & 3 & 5 \\
\hline & ERA5 & 0.665 & 0.736 & 0.808 & 0.587 & 0.808 & 11.98 & 9.35 & 16.27 & 36.70 & 16.43 & 3 & 1 & 2 & 6 & 3 & 6 & 4 & 4 & 6 & 4 \\
\hline & PGF v3 & 0.670 & 0.728 & 0.807 & 0.620 & 0.808 & 7.68 & 7.42 & 15.47 & 33.23 & 15.28 & 2 & 3 & 3 & 1 & 2 & 1 & 2 & 2 & 2 & 2 \\
\hline
\end{tabular}




\subsection{Boxplots}

6.2.1 Combined calibration and evaluation periods

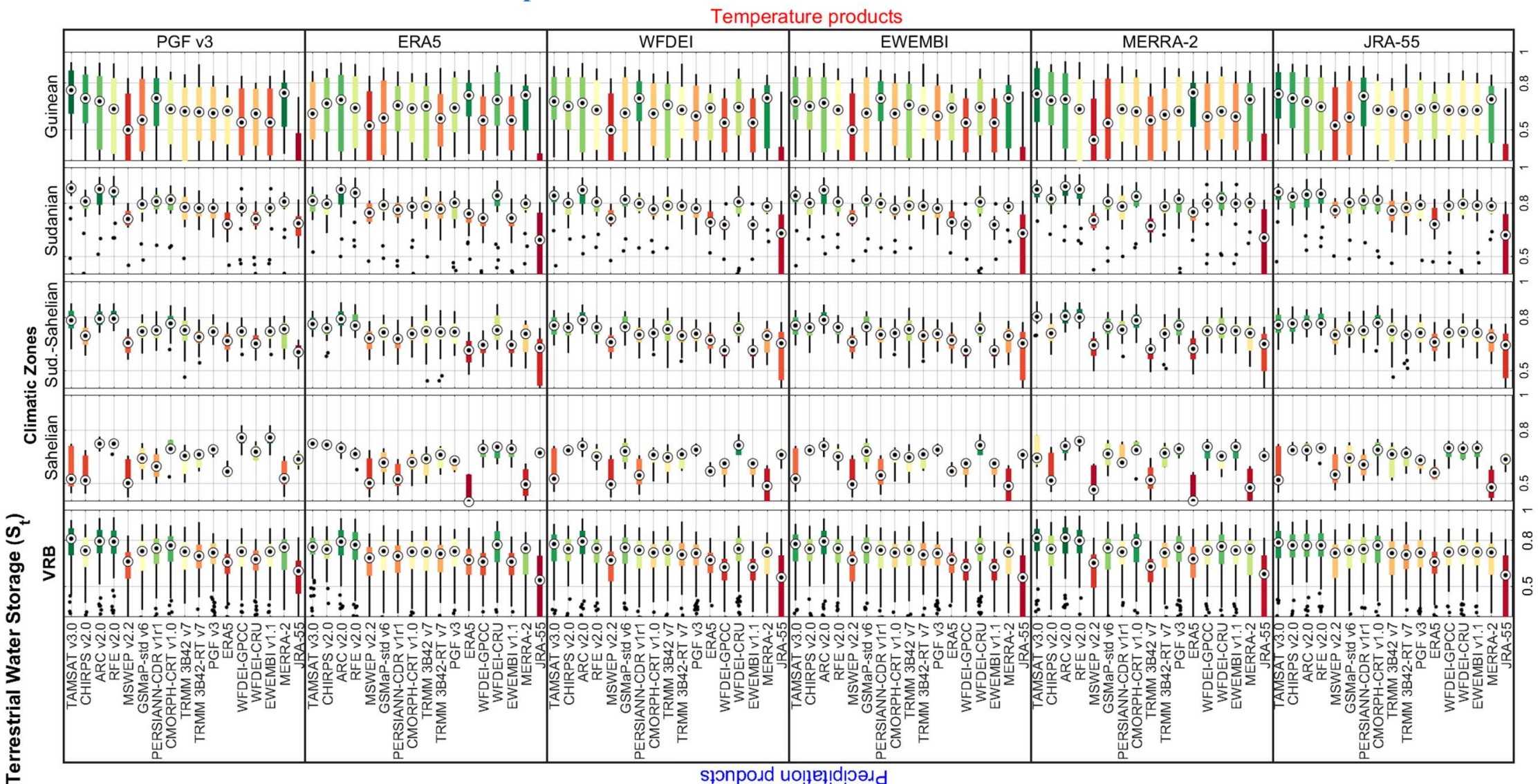

Figure S13. Pearson correlation coefficient $(r)$ of terrestrial water storage (St) over the entire simulation period (2003-2012) for the Volta River basin (VRB) and its climatic zones, using different combinations of precipitation and temperature products for hydrological modelling. Each boxplot has 120 values corresponding to the number of months.

The boxplots are colored from the best (green) to the worst performance (red) based on the median value. 


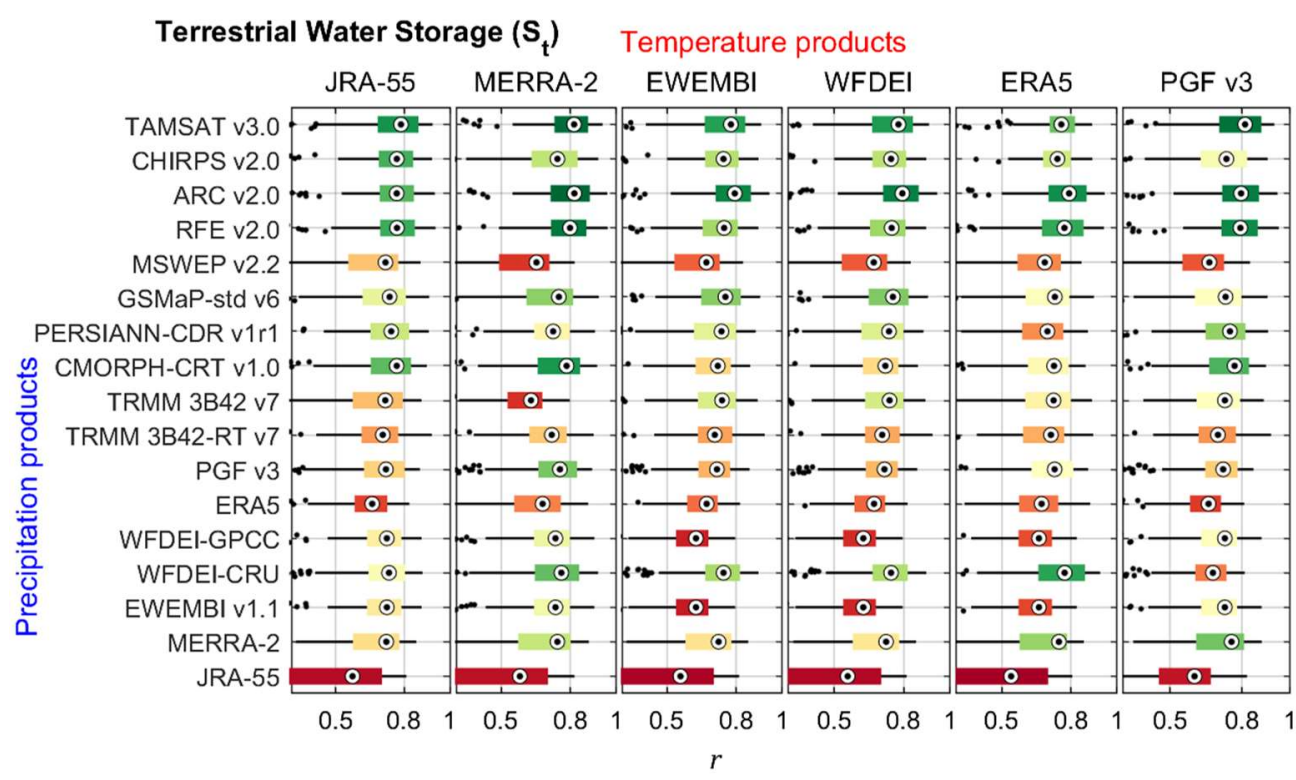

Figure S14. Pearson correlation coefficient $(r)$ of terrestrial water storage $(S t)$ over the entire simulation period (2003-2012) for the Volta River basin (VRB), using different combinations of precipitation and temperature products for hydrological modelling. Each boxplot has 104 values representing the combined performance of the calibration and evaluation periods for 52 pixels. The boxplots are colored from the best (green) to the worst performance (red) based on the median value.

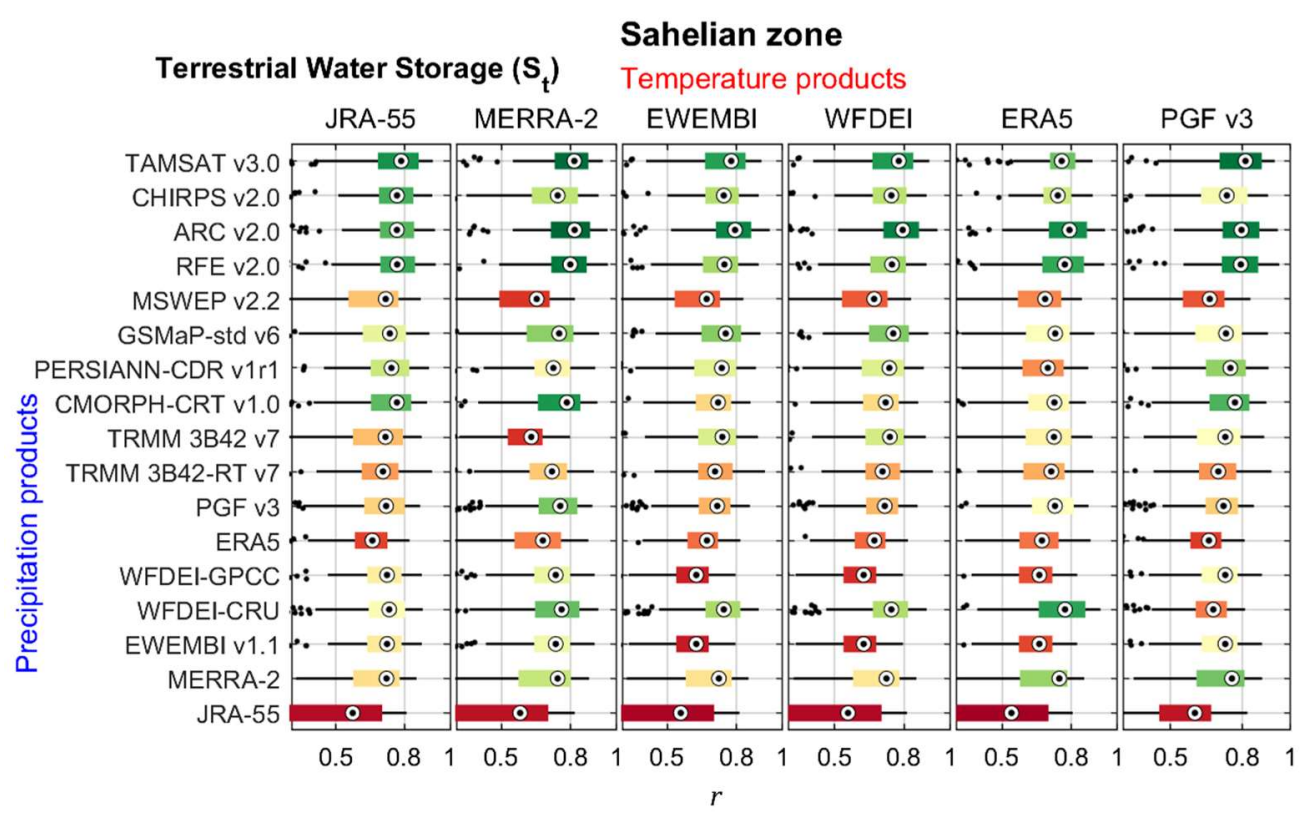

Figure S15. Pearson correlation coefficient $(r)$ of terrestrial water storage $(S t)$ over the entire simulation period (2003-2012) for the Sahelian zone, using different combinations of precipitation and temperature products for hydrological modelling. The boxplots are colored from the best (green) to the worst performance (red) based on the median value. 


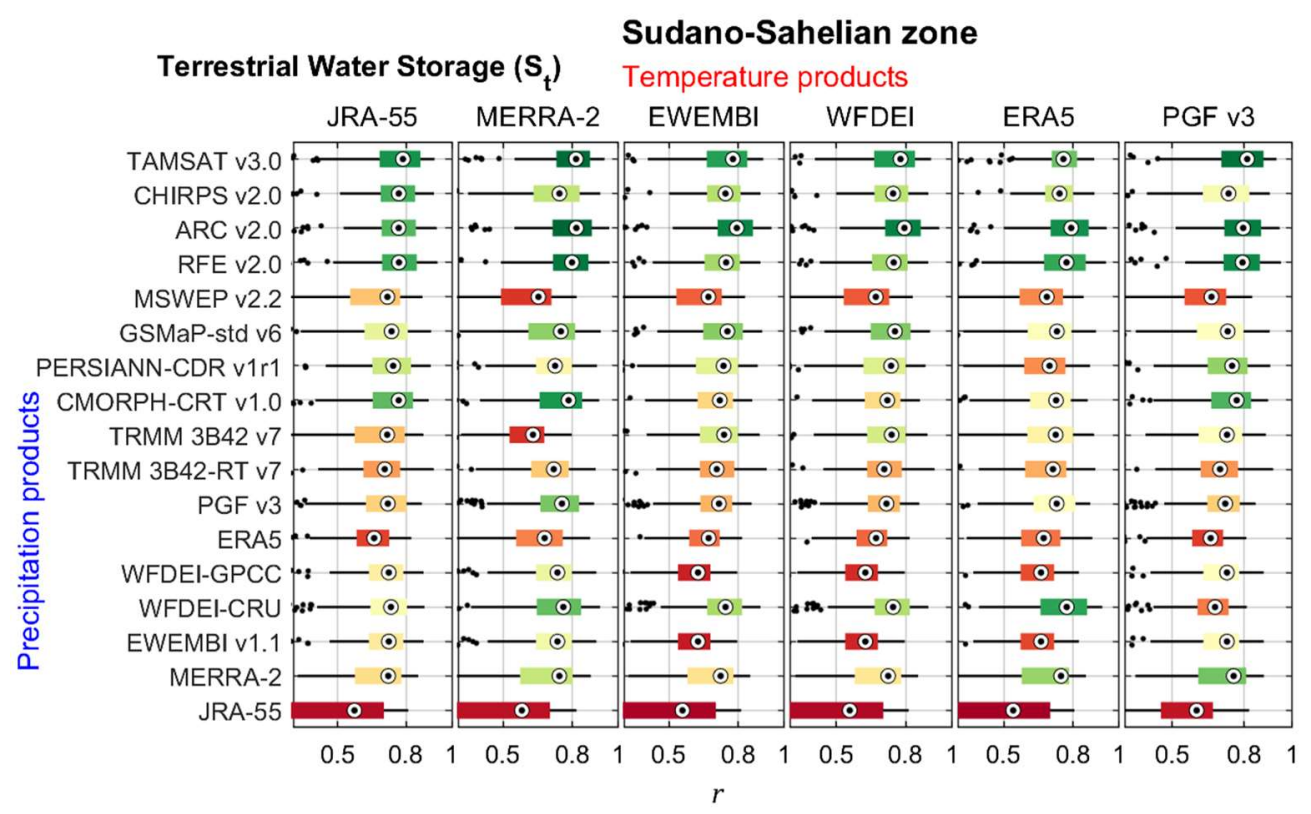

Figure S16. Pearson correlation coefficient $(r)$ of terrestrial water storage $(S t)$ over the entire simulation period (2003-2012) for the Sudano-Sahelian zone, using different combinations of precipitation and temperature products for hydrological modelling. The boxplots are colored from the best (green) to the worst performance (red) based on the median value.

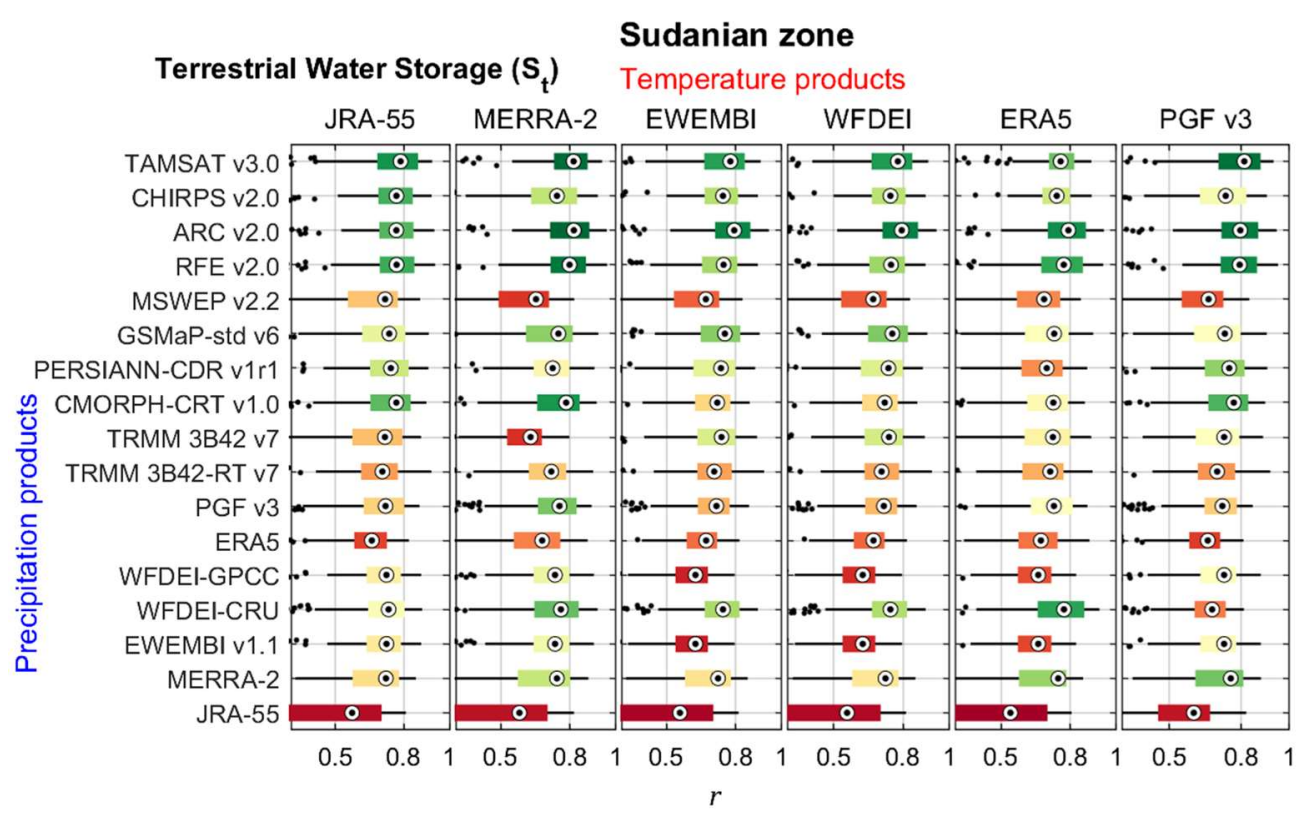

Figure S17. Pearson correlation coefficient $(r)$ of terrestrial water storage $(S t)$ over the entire simulation period (2003-2012) for the Sudanian zone, using different combinations of precipitation and temperature products for hydrological modelling. The boxplots are colored from the best (green) to the worst performance (red) based on the median value. 


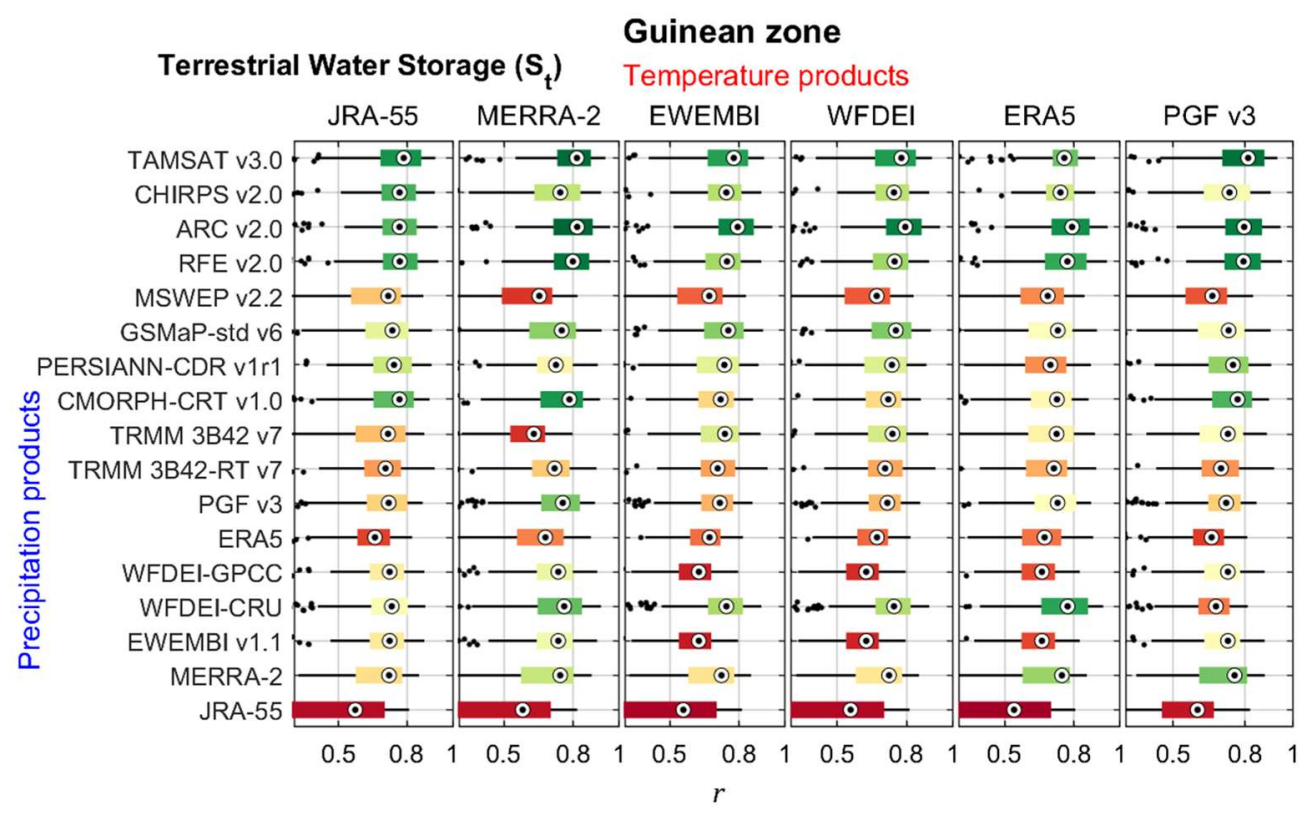

Figure S18. Pearson correlation coefficient $(r)$ of terrestrial water storage $(S t)$ over the entire simulation period (2003-2012) for the Guinean zone, using different combinations of precipitation and temperature products for hydrological modelling. The boxplots are colored from the best (green) to the worst performance (red) based on the median value. 


\subsubsection{Separated calibration and evaluation periods}
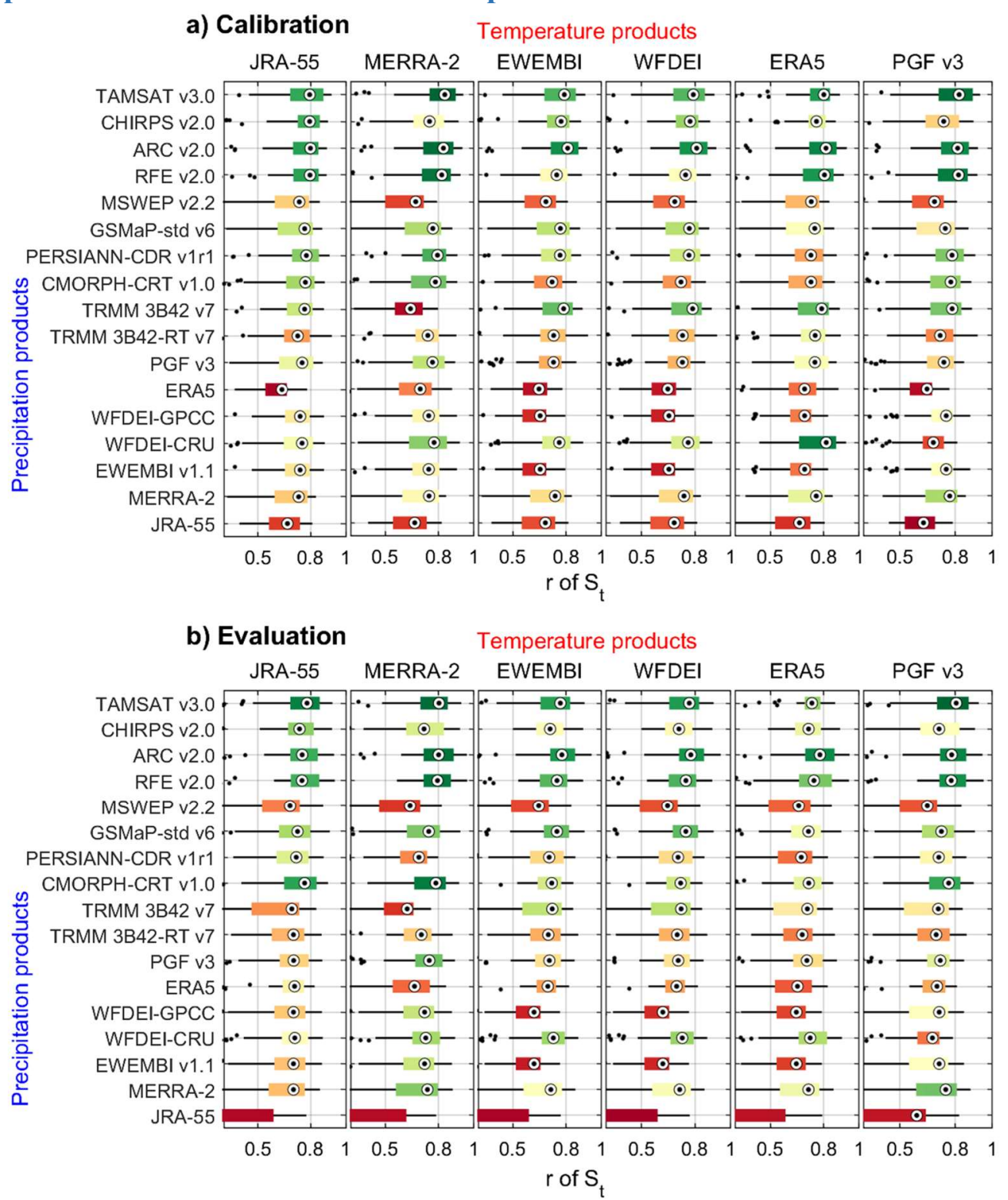

Figure S19. Pearson correlation coefficient ( $r$ ) of terrestrial water storage (St) over (a) the calibration (2003-2008) and (b) the evaluation (2009-2012) periods for the Volta River basin (VRB), using different combinations of precipitation and temperature products for hydrological modelling. The boxplots are colored from the best (green) to the worst performance (red) based on the median value. 

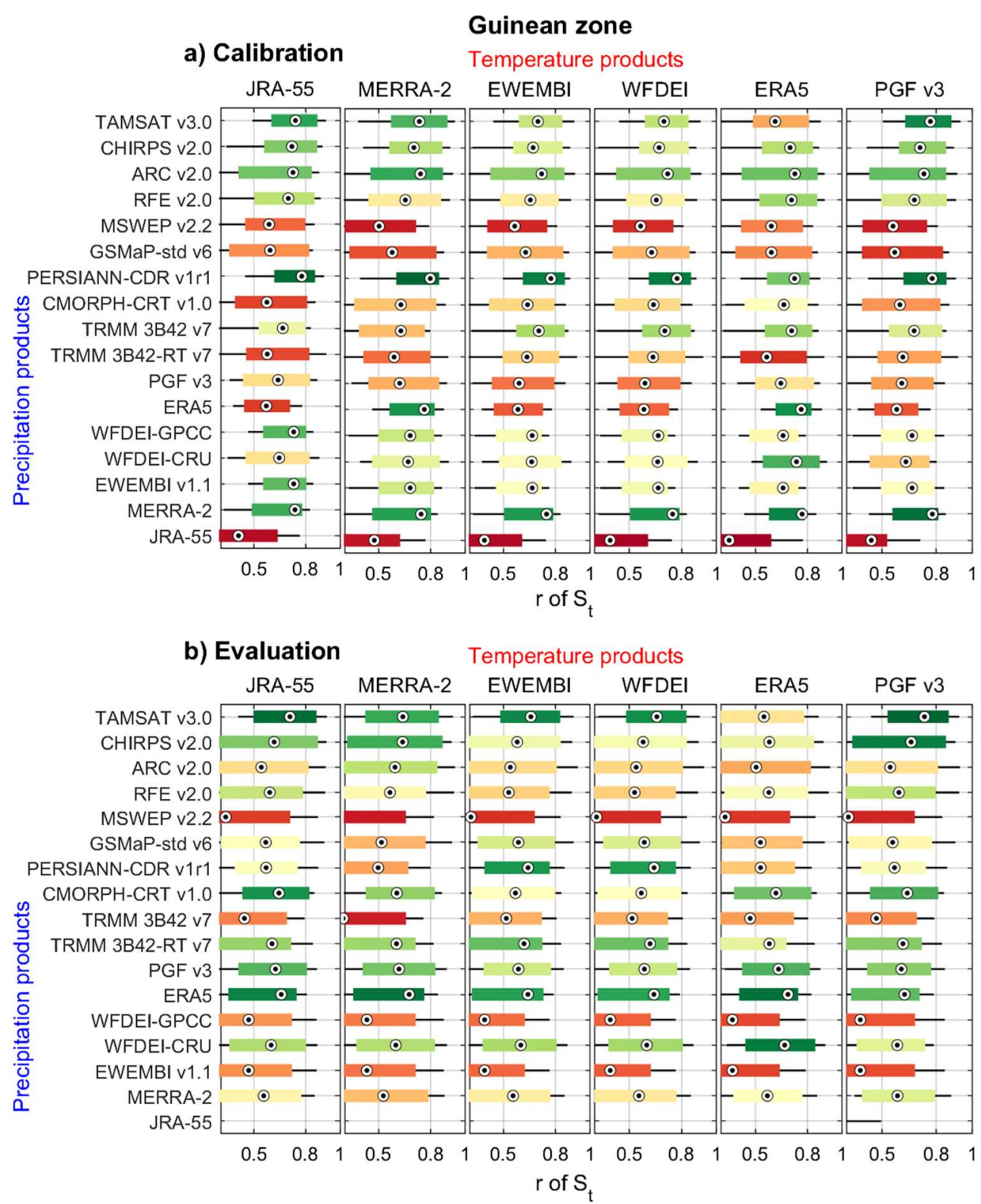

Figure S20. Pearson correlation coefficient $(r)$ of terrestrial water storage (St) over (a) the calibration (2003-2008) and (b) the evaluation (2009-2012) periods for the Guinean zone, using different combinations of precipitation and temperature products for hydrological modelling. The boxplots are colored from the best (green) to the worst performance (red) based on the median value. 

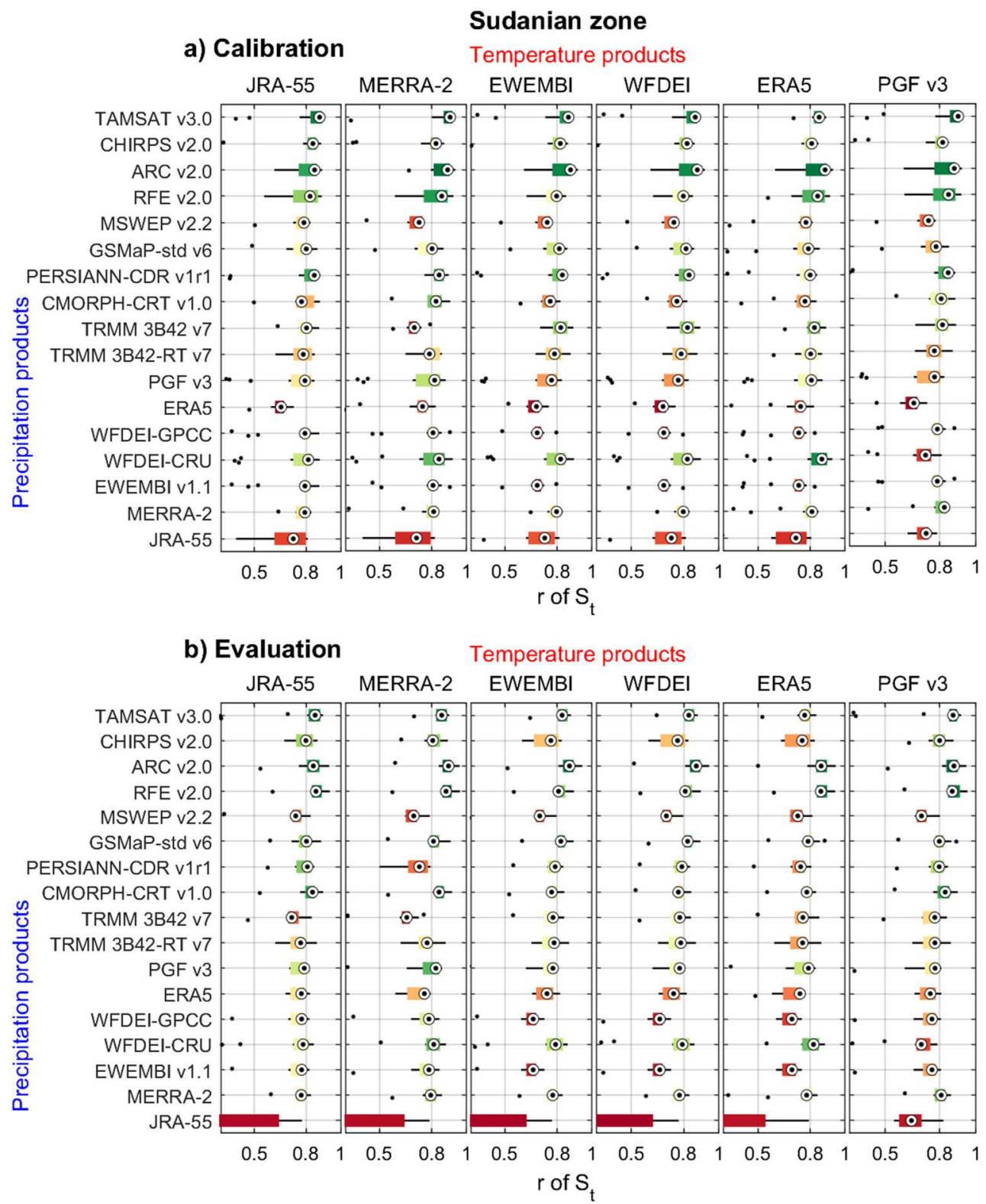

Figure S21. Pearson correlation coefficient $(r)$ of terrestrial water storage (St) over (a) the calibration (2003-2008) and (b) the evaluation (2009-2012) periods for the Sudanian zone, using different combinations of precipitation and temperature products for hydrological modelling. The boxplots are colored from the best (green) to the worst performance (red) based on the median value. 

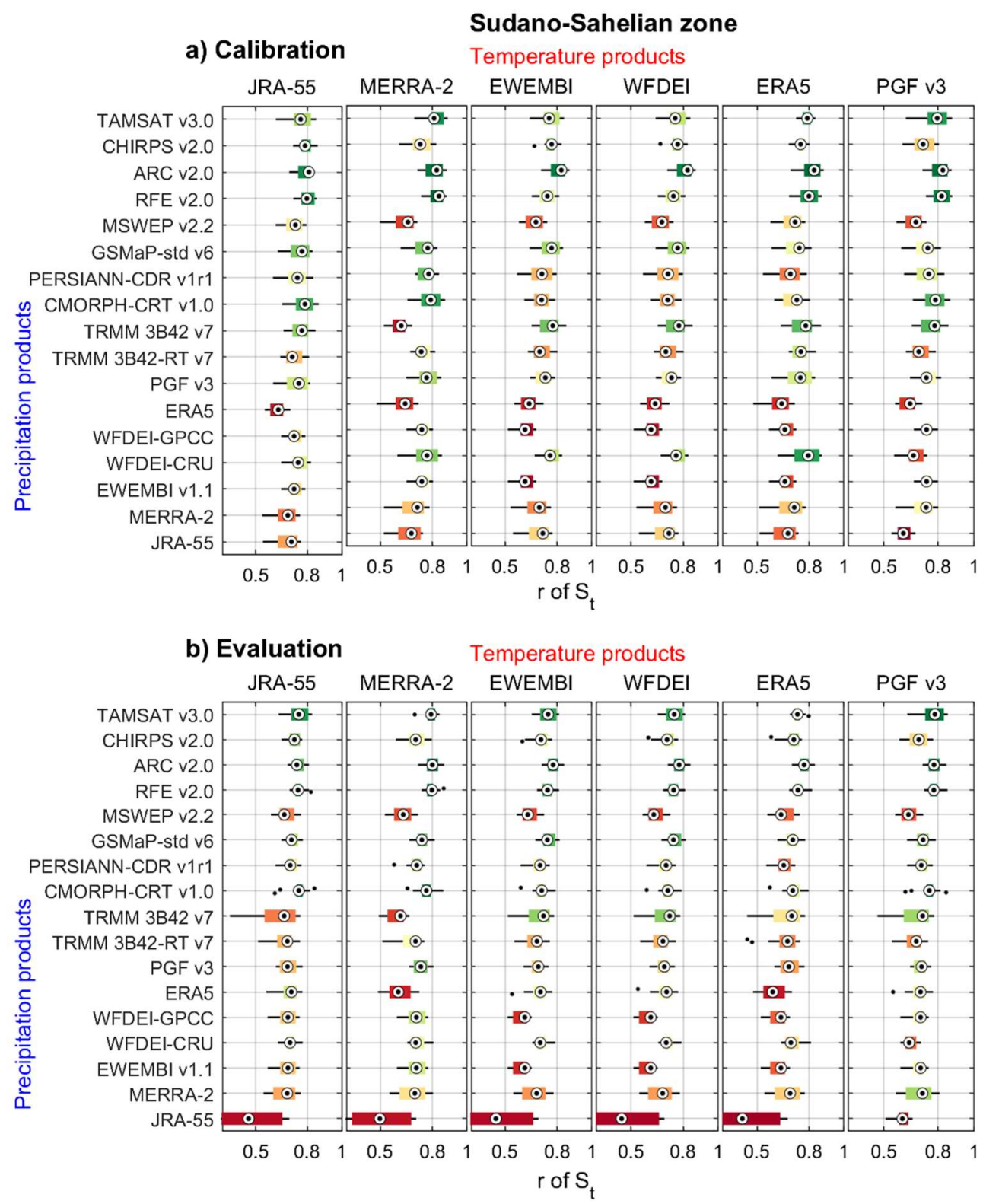

Figure S22. Pearson correlation coefficient $(r)$ of terrestrial water storage (St) over (a) the calibration (2003-2008) and (b) the evaluation (2009-2012) periods for the Sudano-Sahelian zone, using different combinations of precipitation and temperature products for hydrological modelling. The boxplots are colored from the best (green) to the worst performance (red) based on the median value. 

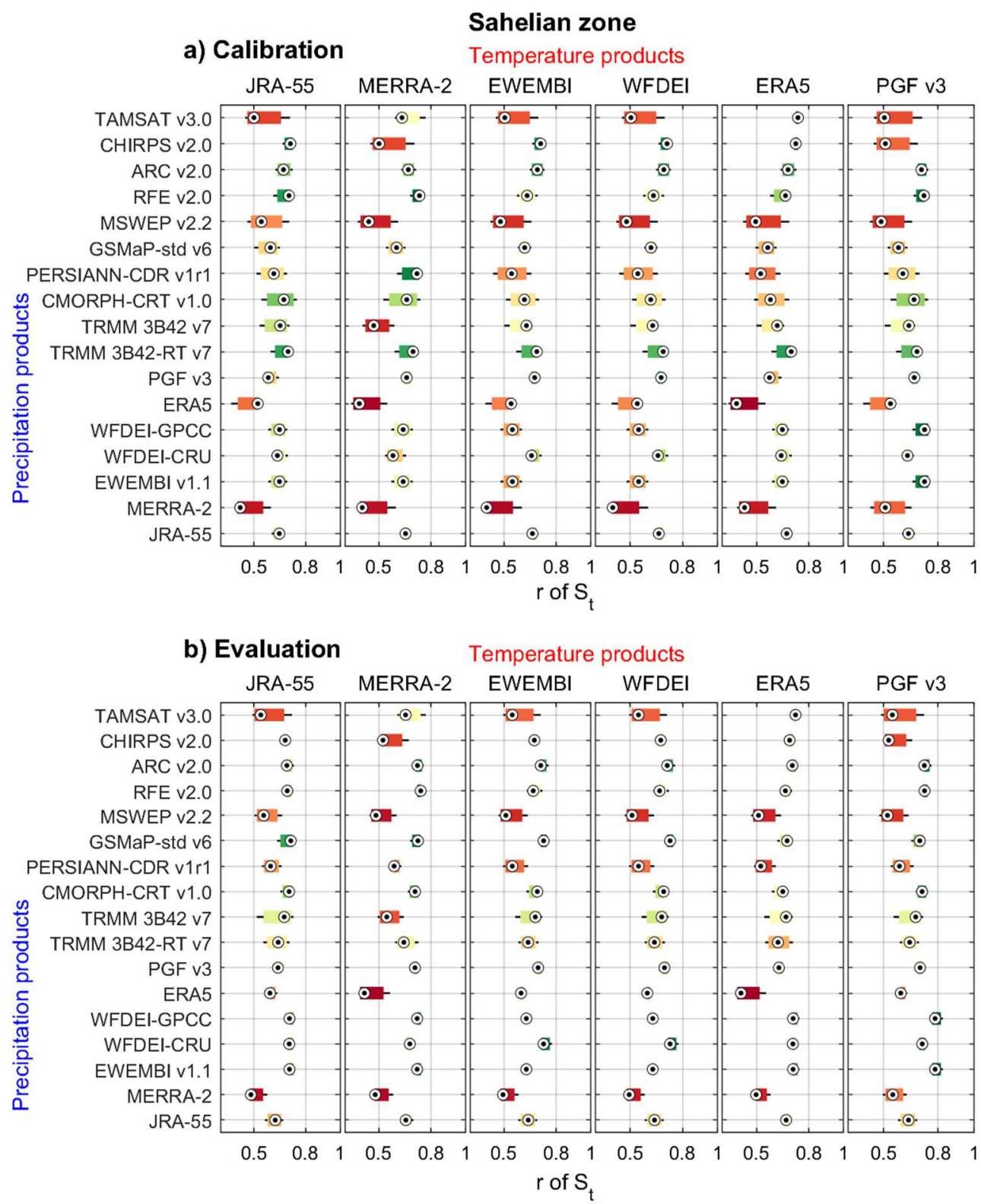

Figure S23. Pearson correlation coefficient $(r)$ of terrestrial water storage (St) over (a) the calibration (2003-2008) and (b) the evaluation (2009-2012) periods for the Sahelian zone, using different combinations of precipitation and temperature products for hydrological modelling. The boxplots are colored from the best (green) to the worst performance (red) based on the median value. 
6.3 Time series

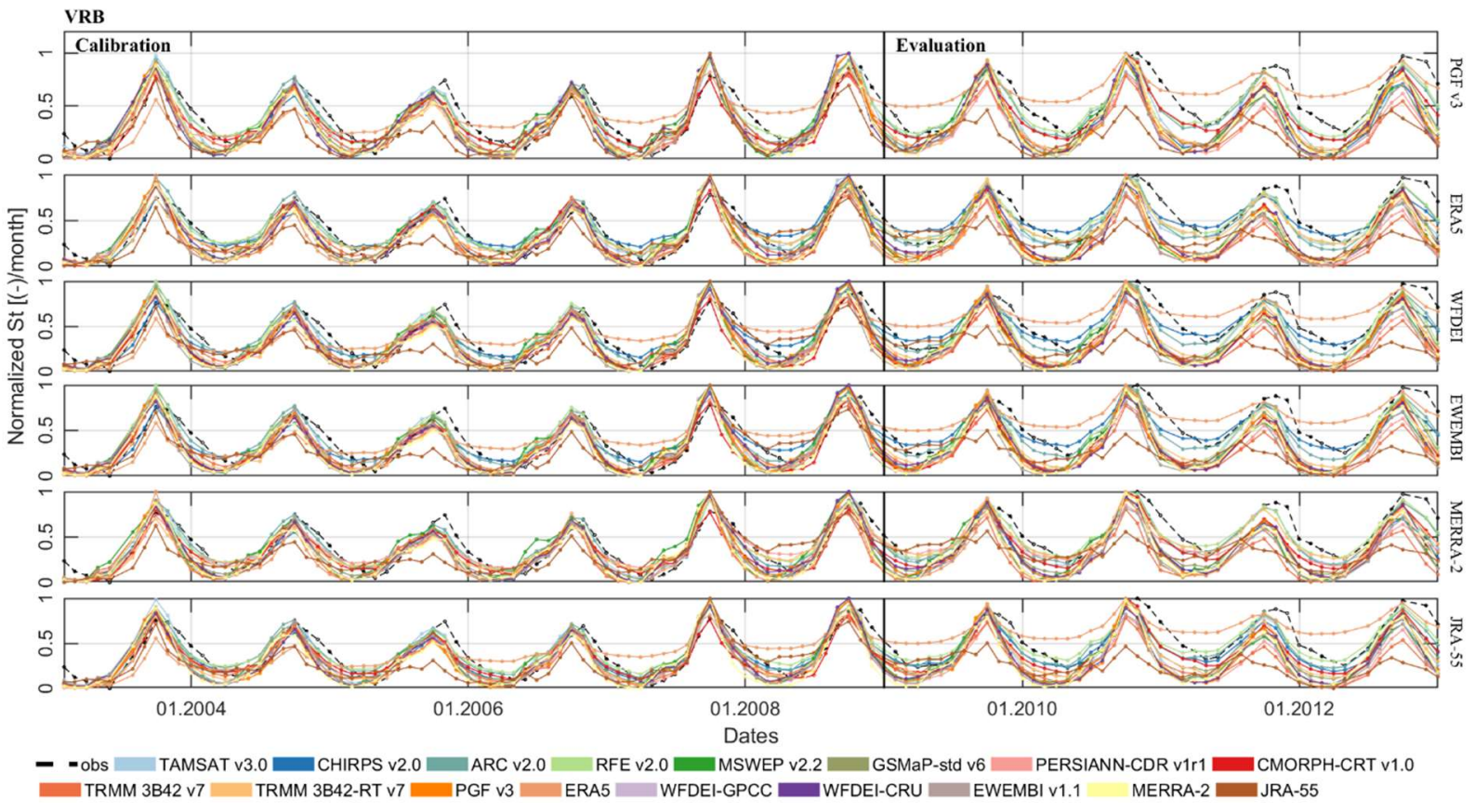

Figure S24. Monthly time series of terrestrial water storage (St) in the Volta River basin (VRB) obtained for different combinations of rainfall datasets (colors in legend) and temperature datasets (right y-axis of the 6 subplots) used as inputs in a hydrological model (i.e. mHM). All values are normalized between 0 and 1.

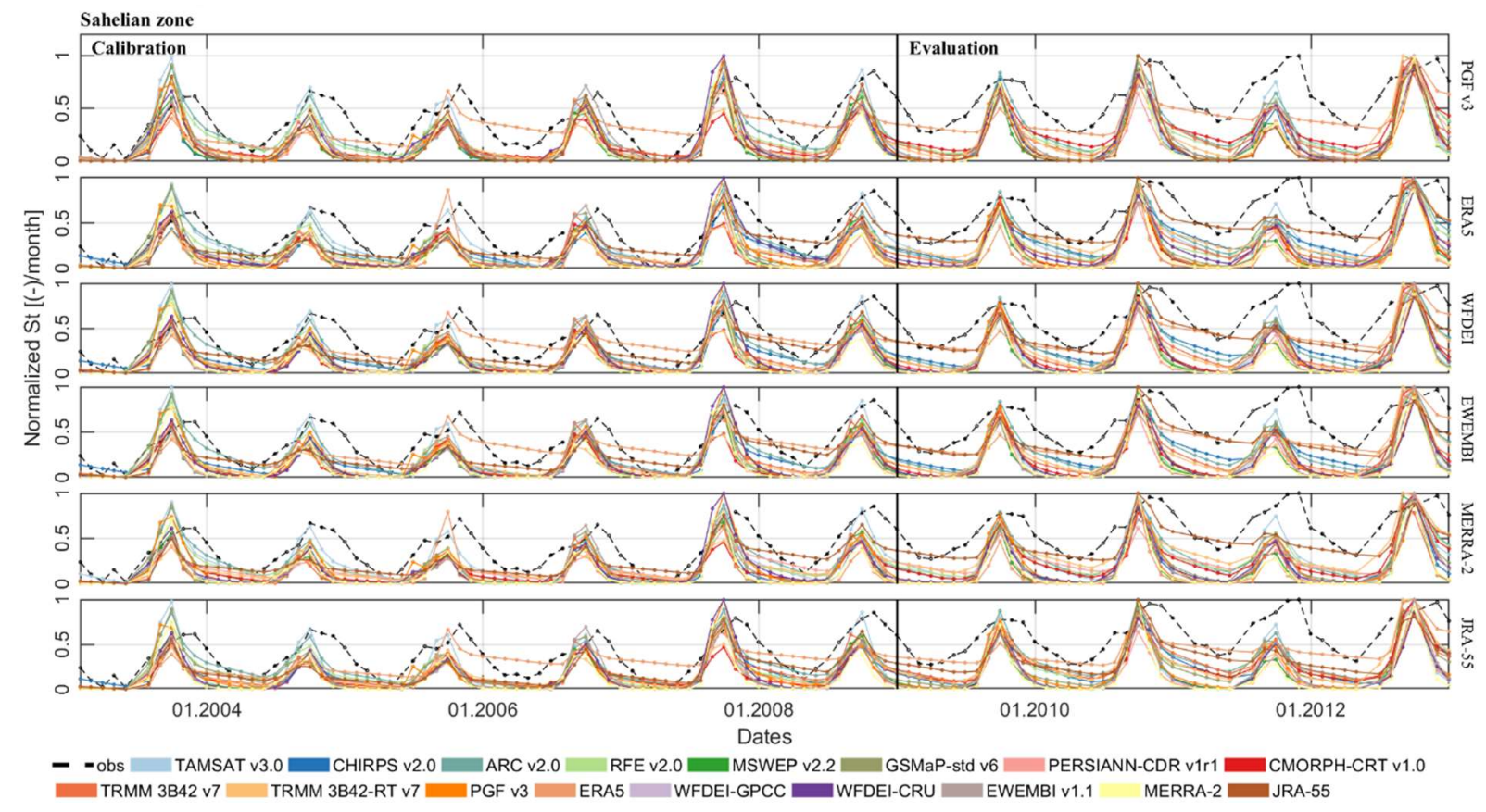

Figure S25. Monthly time series of terrestrial water storage (St) in the Sahelian zone of the Volta basin obtained for different combinations of rainfall datasets (colors in legend) and temperature datasets (right y-axis of the 6 subplots) used as inputs in a hydrological model (i.e. mHM). All values are normalized between 0 and 1. 


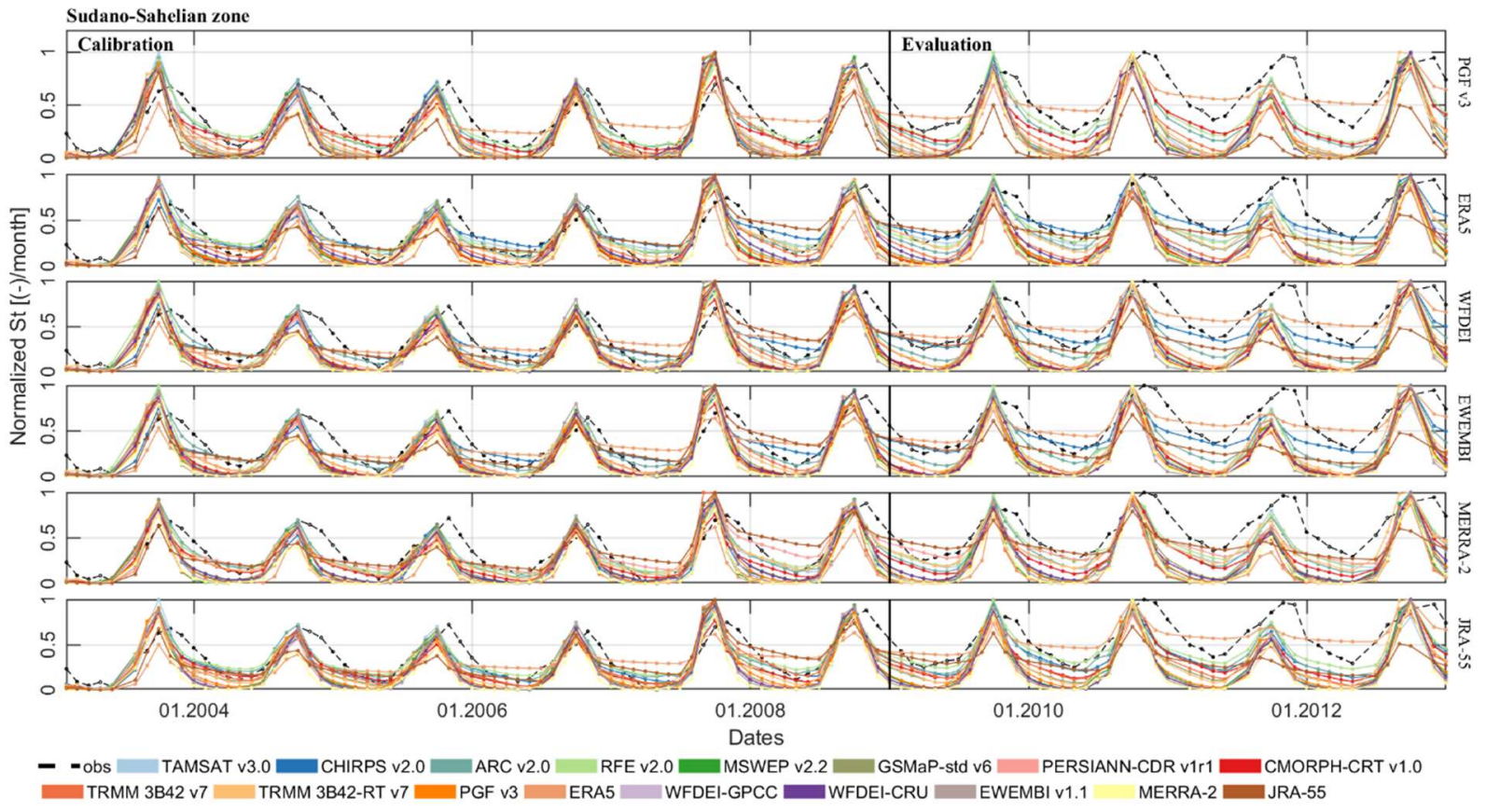

Figure S26. Monthly time series of terrestrial water storage (St) in the Sudano-Sahelian zone of the Volta basin obtained for different combinations of rainfall datasets (colors in legend) and temperature datasets (right y-axis of the 6 subplots) used as inputs in a hydrological model (i.e. mHM). All values are normalized between 0 and 1.

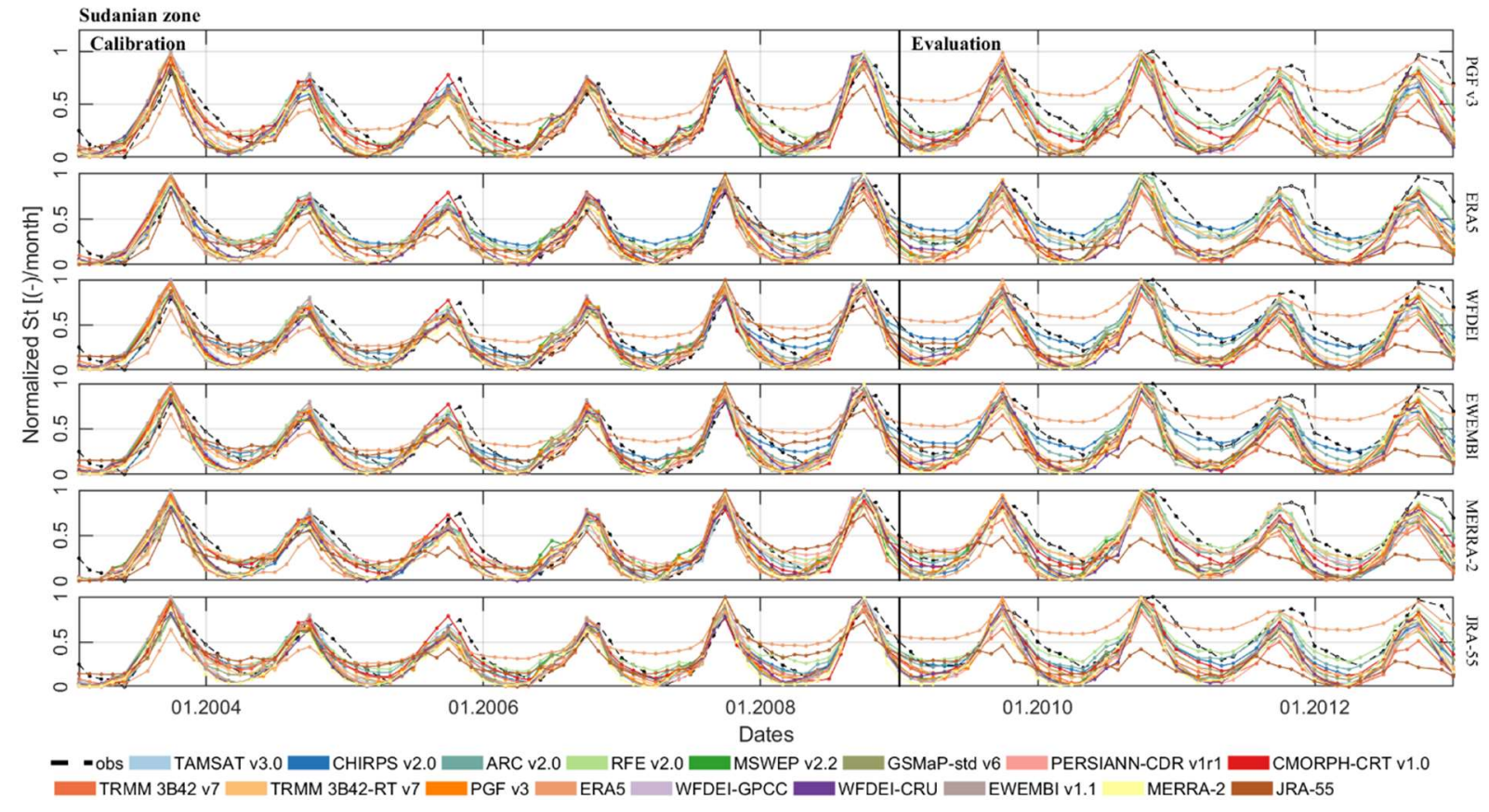

Figure S27. Monthly time series of terrestrial water storage (St) in the Sudanian zone of the Volta basin obtained for different combinations of rainfall datasets (colors in legend) and temperature datasets (right y-axis of the 6 subplots) used as inputs in a hydrological model (i.e. mHM). All values are normalized between 0 and 1. 


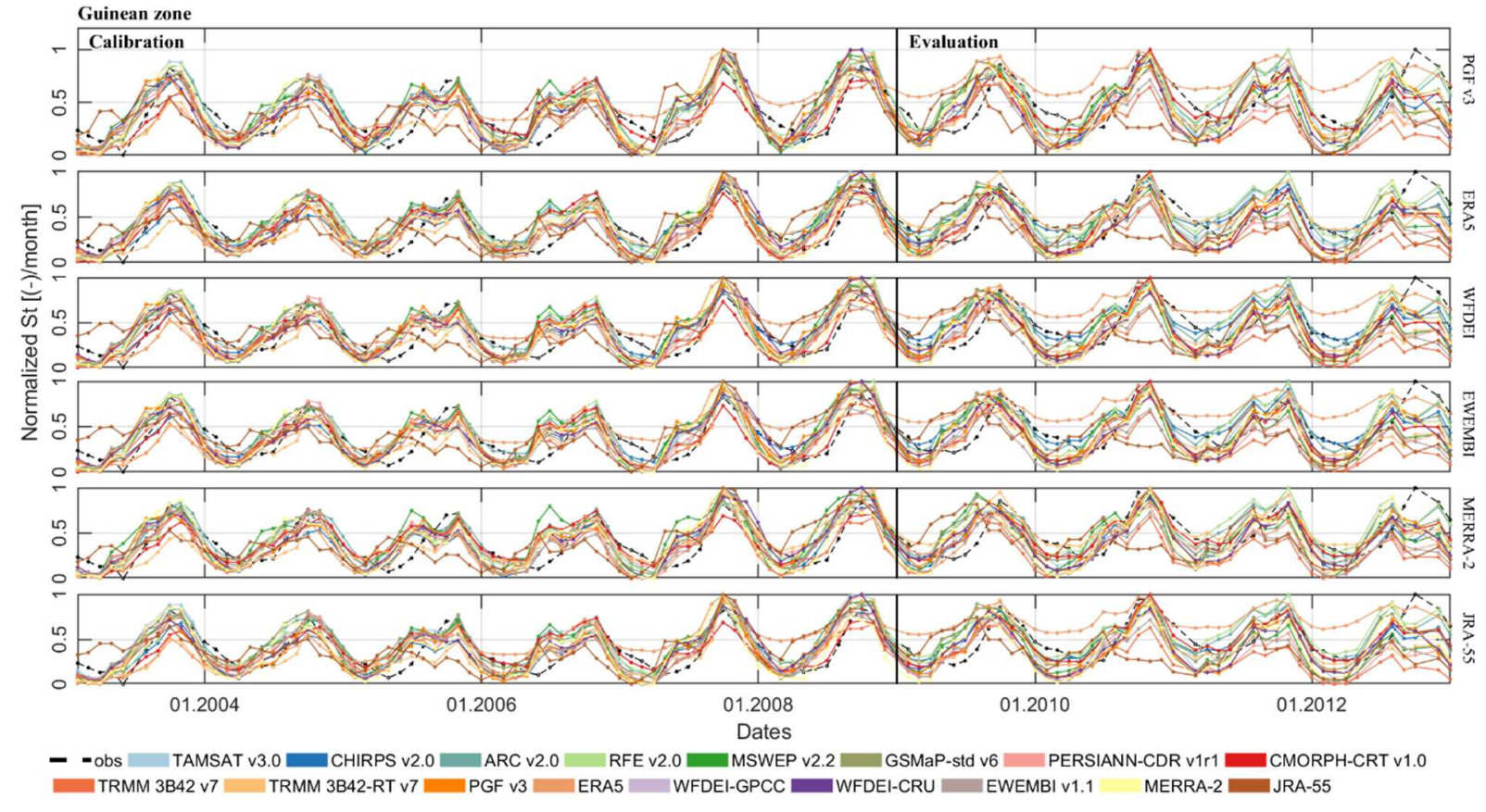

Figure S28. Monthly time series of terrestrial water storage (St) in the Guinean zone of the Volta basin obtained for different combinations of rainfall datasets (colors in legend) and temperature datasets (right y-axis of the 6 subplots) used as inputs in a hydrological model (i.e. mHM). All values are normalized between 0 and 1. 


\section{Model performance for soil moisture}

\subsection{Ranking of rainfall and temperature datasets}

Table S14. Ranking of rainfall and temperature datasets based on the Pearson correlation coefficient $(r)$ of soil moisture $\left(S_{\mathrm{u}}\right)$ over the simulation period. The mean and the second-order coefficient of variation $\left(V_{2}\right)$ are calculated across 6 temperature datasets for each rainfall dataset, and across 17 rainfall datasets for each temperature dataset. The rank is given from the best (green) to the worst performance (red) for 4 climatic zones in the Volta River basin (VRB)

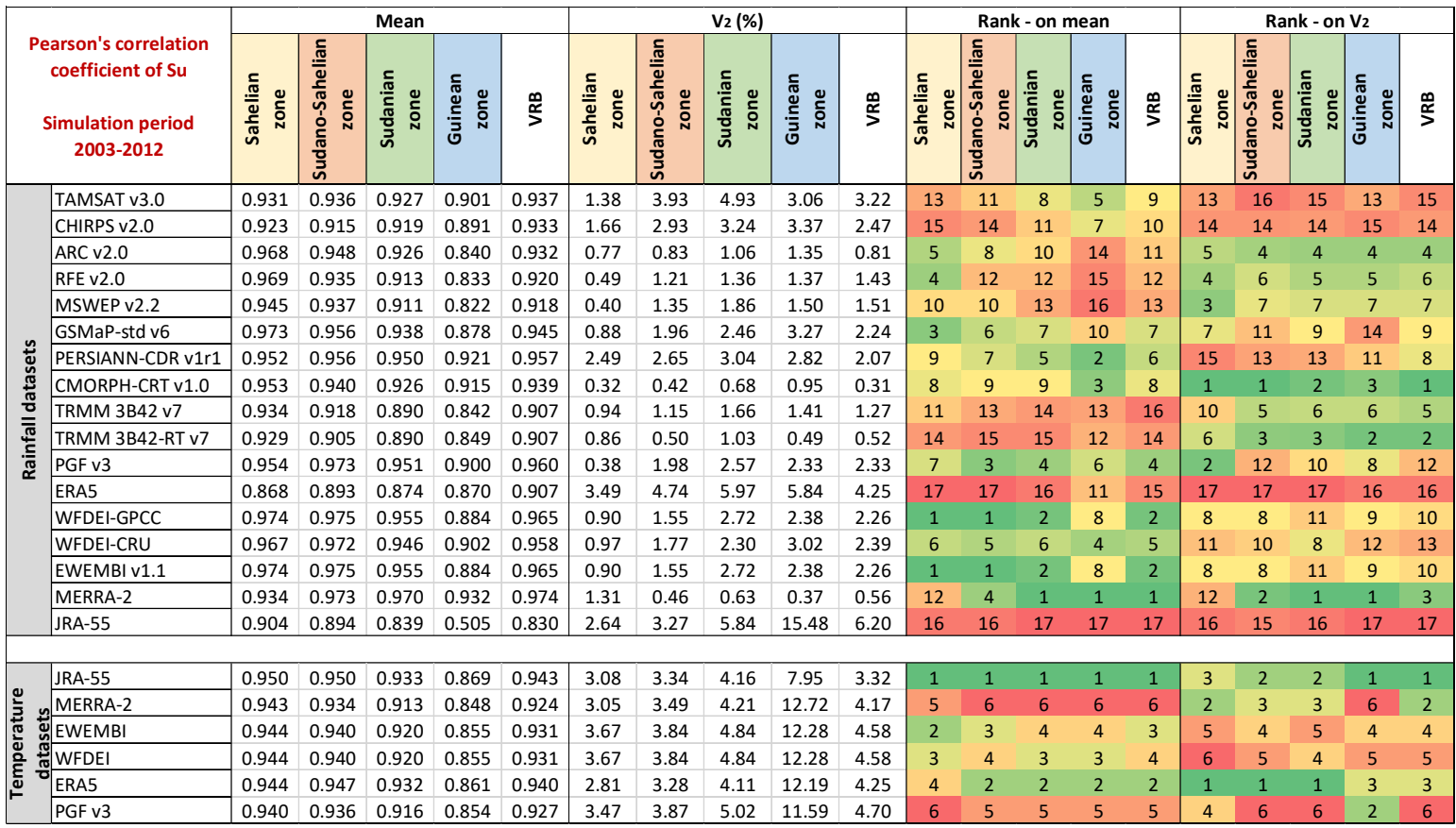

Table S15. Ranking of rainfall and temperature datasets based on the spatial pattern efficiency $\left(E_{\mathrm{SP}}\right)$ of soil moisture $\left(S_{\mathrm{u}}\right)$ over the simulation period. The mean and the second-order coefficient of variation $\left(V_{2}\right)$ are calculated across 6 temperature datasets for each rainfall dataset, and across 17 rainfall datasets for each temperature dataset. The rank is given from the best (green) to the worst performance (red) for 4 climatic zones in the Volta River basin (VRB)

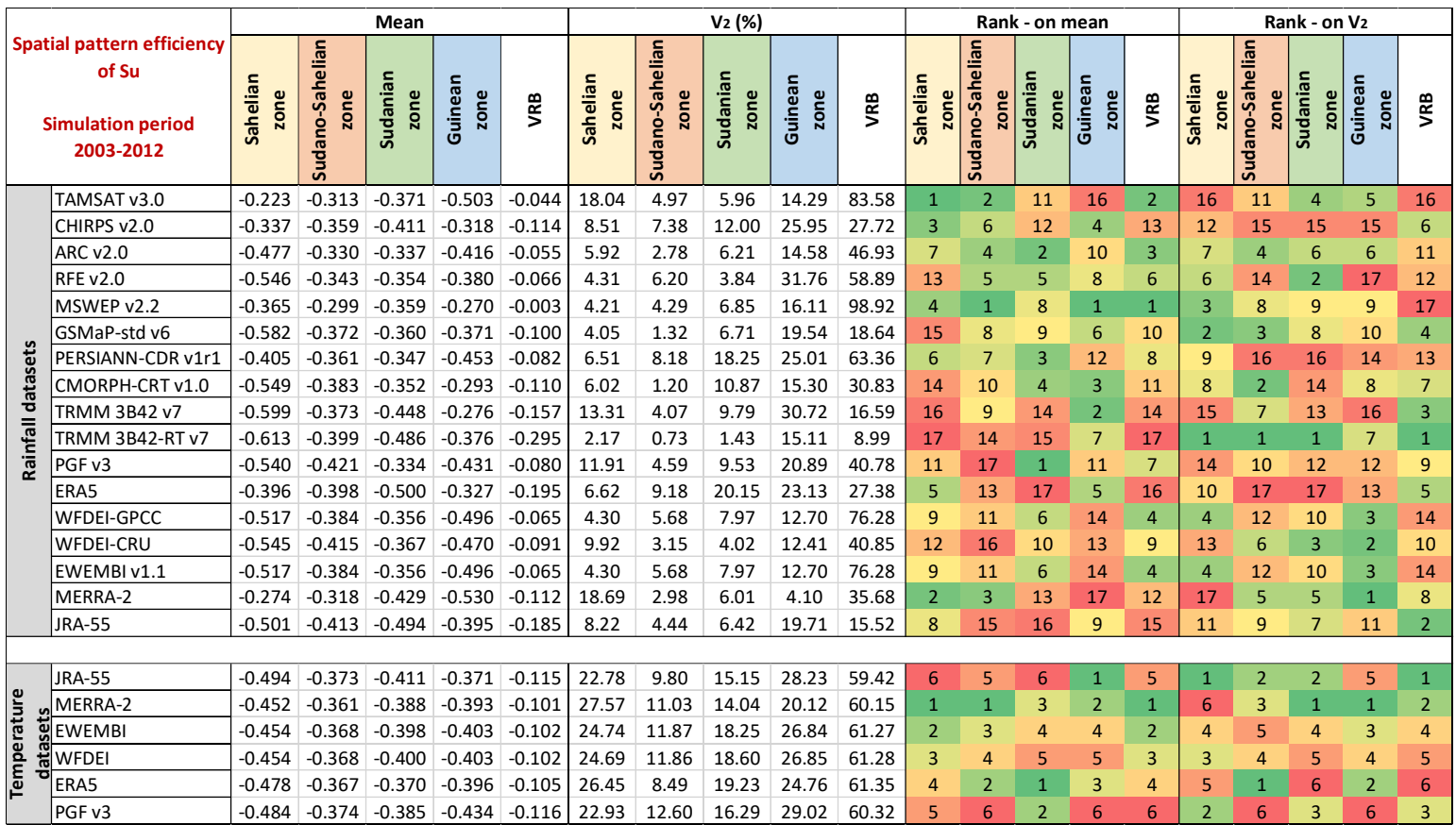




\subsection{Boxplots}

7.2.1 Combined calibration and evaluation periods

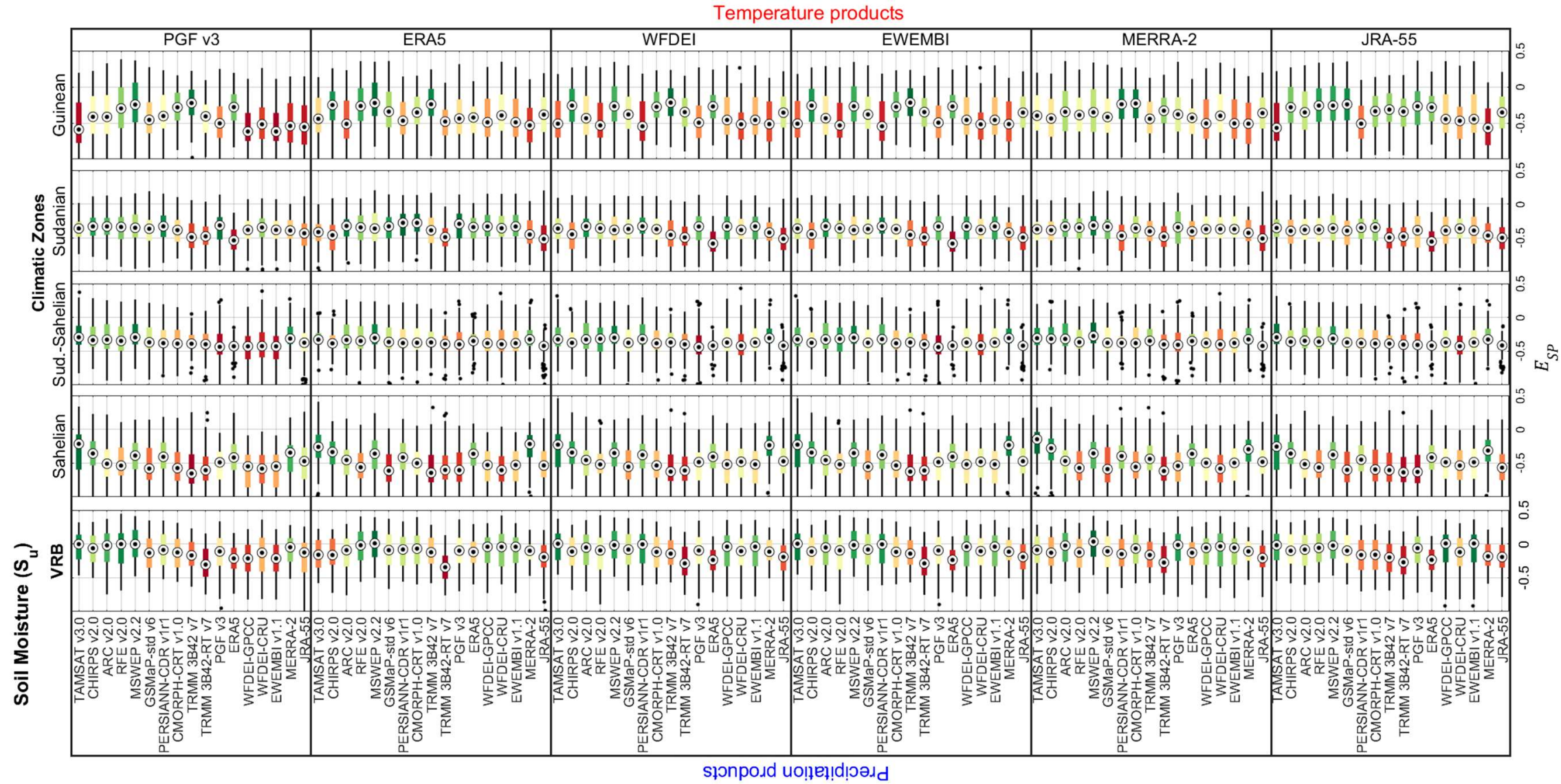

Figure S29. Spatial pattern efficiency $\left(E_{\mathrm{SP}}\right)$ of soil moisture $\left(S_{\mathrm{u}}\right)$ over the entire simulation period (2003-2012) for the Volta River basin (VRB) and its climatic zones, using different combinations of precipitation and temperature products for hydrological modelling. Each boxplot has 120 values corresponding to the number of months. The boxplots are colored from the best (green) to the worst performance (red) based on the median value. 


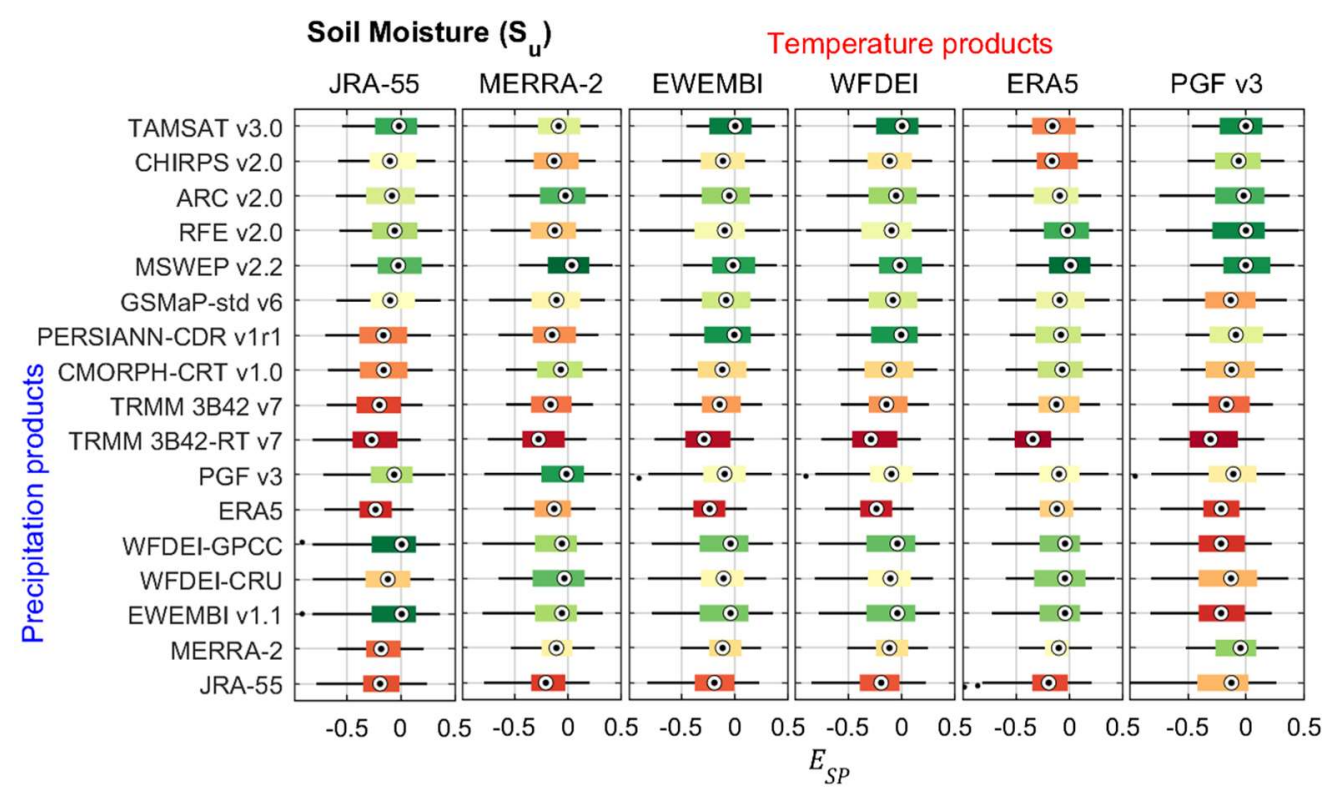

Figure S30. Spatial pattern efficiency $\left(E_{\mathrm{SP}}\right)$ of soil moisture $\left(S_{\mathrm{u}}\right)$ over the entire simulation period (2003-2012) for the Volta River basin (VRB), using different combinations of precipitation and temperature products for hydrological modelling. The boxplots are colored from the best (green) to the worst performance (red) based on the median value.

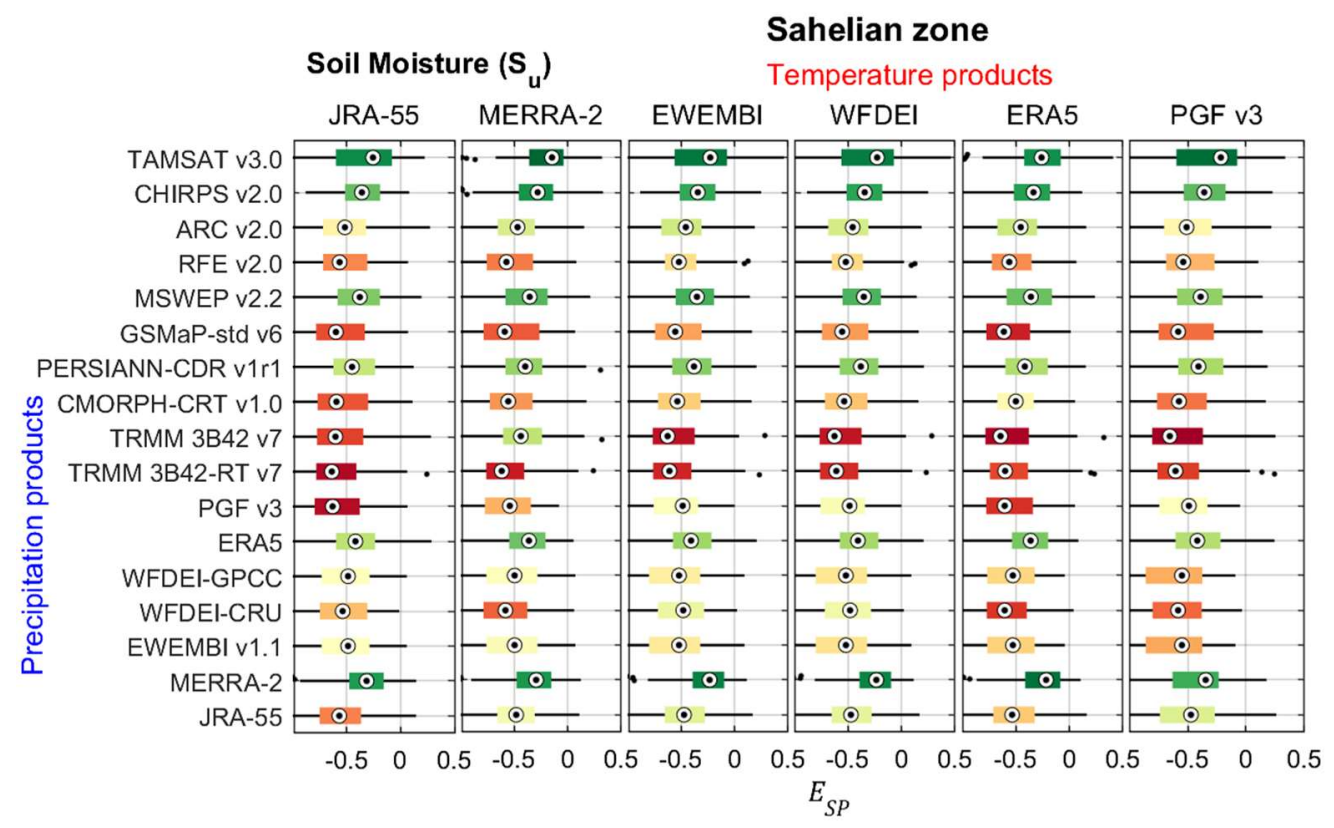

Figure S31. Spatial pattern efficiency $\left(E_{\mathrm{SP}}\right)$ of soil moisture $\left(S_{\mathrm{u}}\right)$ over the entire simulation period (2003-2012) for the Sahelian zone, using different combinations of precipitation and temperature products for hydrological modelling. The boxplots are colored from the best (green) to the worst performance (red) based on the median value. 


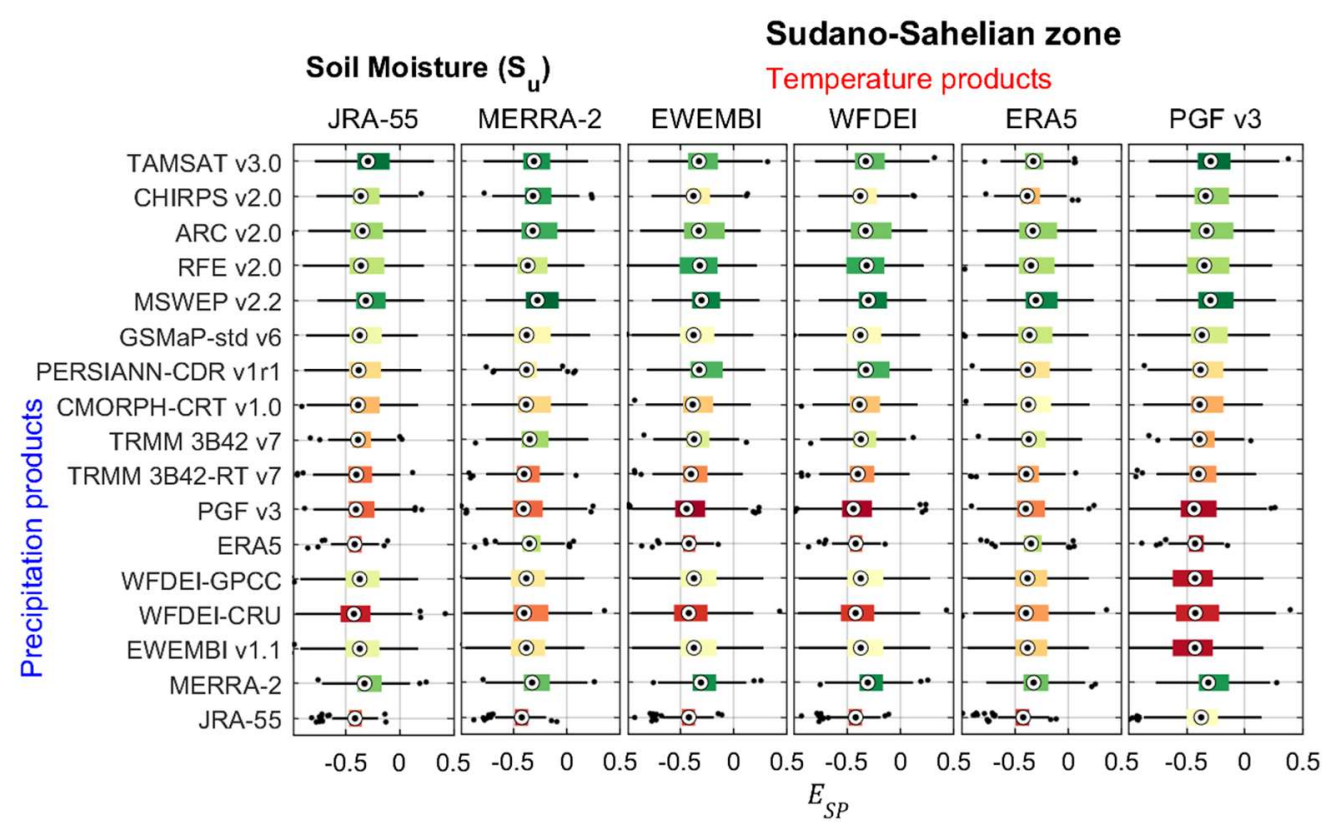

Figure S32. Spatial pattern efficiency $\left(E_{\mathrm{SP}}\right)$ of soil moisture $\left(S_{\mathrm{u}}\right)$ over the entire simulation period (2003-2012) for the Sudano-Sahelian zone, using different combinations of precipitation and temperature products for hydrological modelling. The boxplots are colored from the best (green) to the worst performance (red) based on the median value.

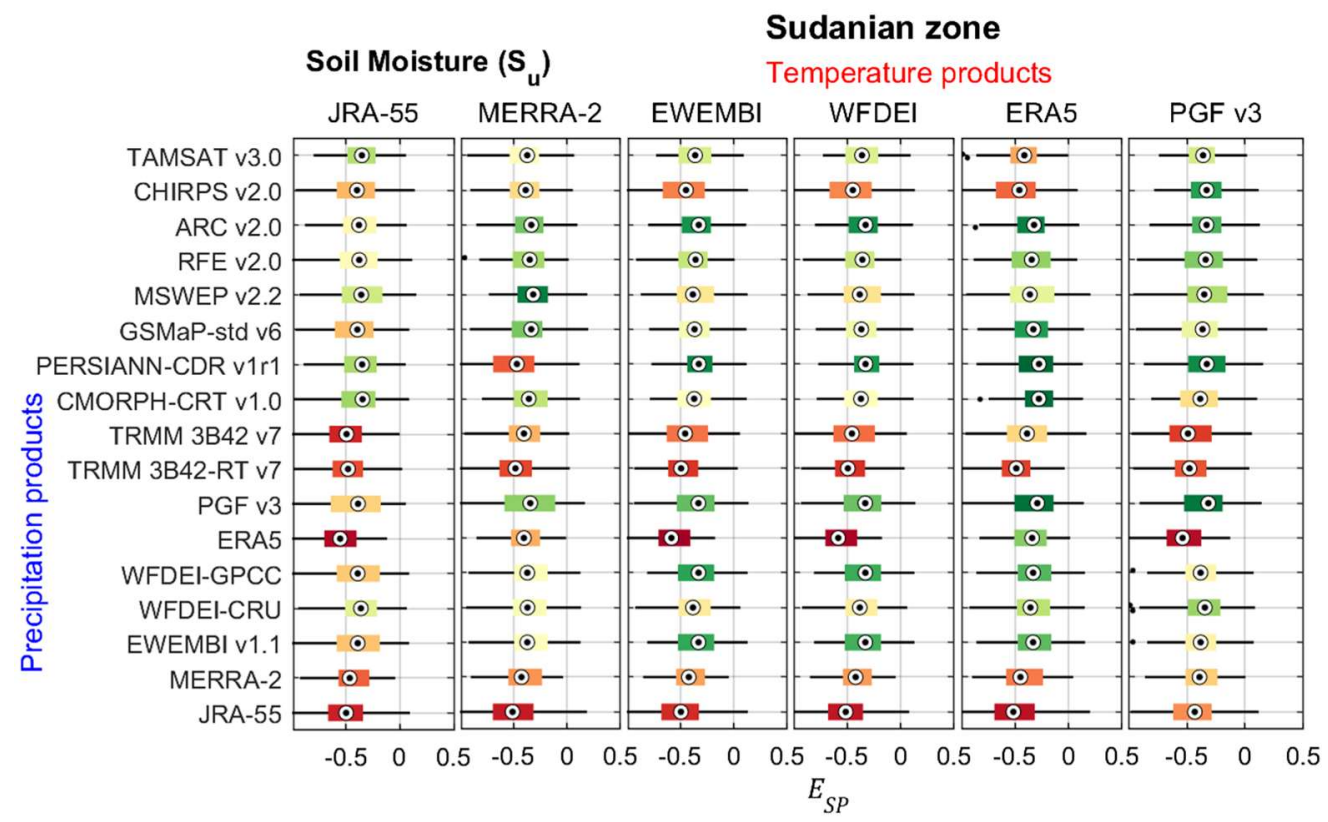

Figure S33. Spatial pattern efficiency $\left(E_{\mathrm{SP}}\right)$ of soil moisture $\left(S_{\mathrm{u}}\right)$ over the entire simulation period (2003-2012) for the Sudanian zone, using different combinations of precipitation and temperature products for hydrological modelling. The boxplots are colored from the best (green) to the worst performance (red) based on the median value. 


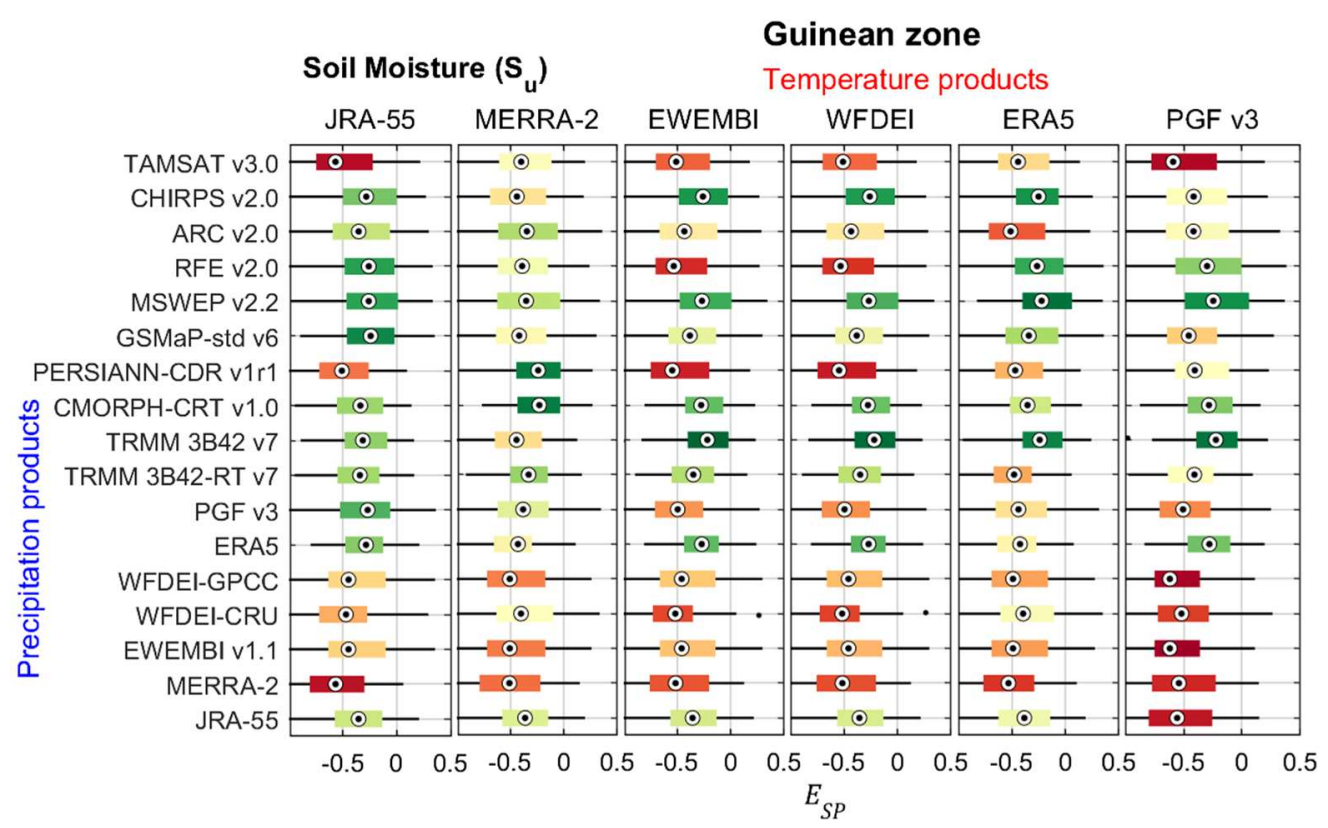

Figure S34. Spatial pattern efficiency $\left(E_{\mathrm{SP}}\right)$ of soil moisture $\left(S_{\mathrm{u}}\right)$ over the entire simulation period (2003-2012) for the Guinean zone, using different combinations of precipitation and temperature products for hydrological modelling. The boxplots are colored from the best (green) to the worst performance (red) based on the median value. 


\subsubsection{Separated calibration and evaluation periods}
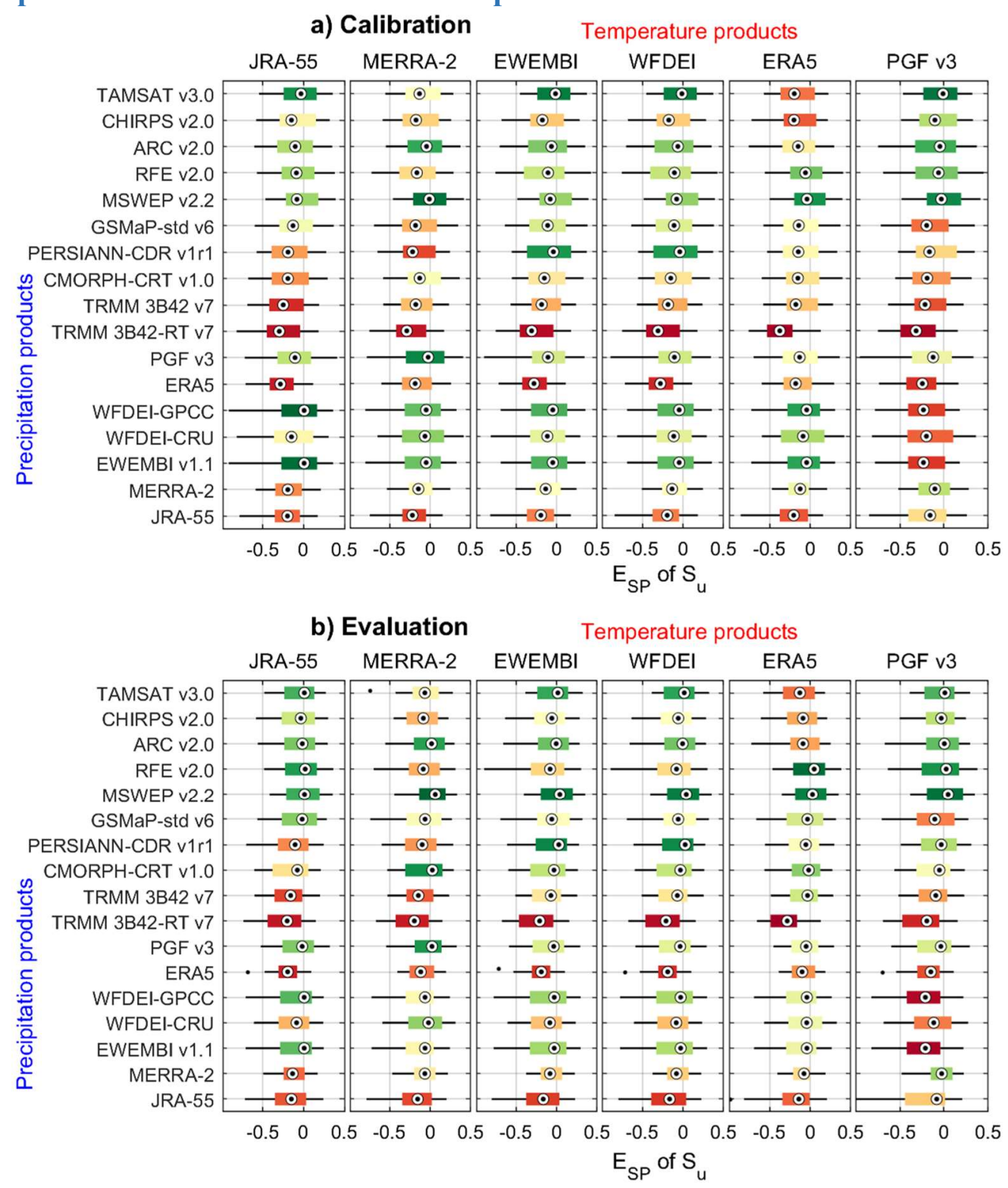

Figure S35. Spatial pattern efficiency $\left(E_{\mathrm{SP}}\right)$ of soil moisture $\left(S_{\mathrm{u}}\right)$ over (a) the calibration (2003-2008) and (b) the evaluation (2009-2012) periods for the Volta River basin (VRB), using different combinations of precipitation and temperature products for hydrological modelling. The boxplots are colored from the best (green) to the worst performance (red) based on the median value. 

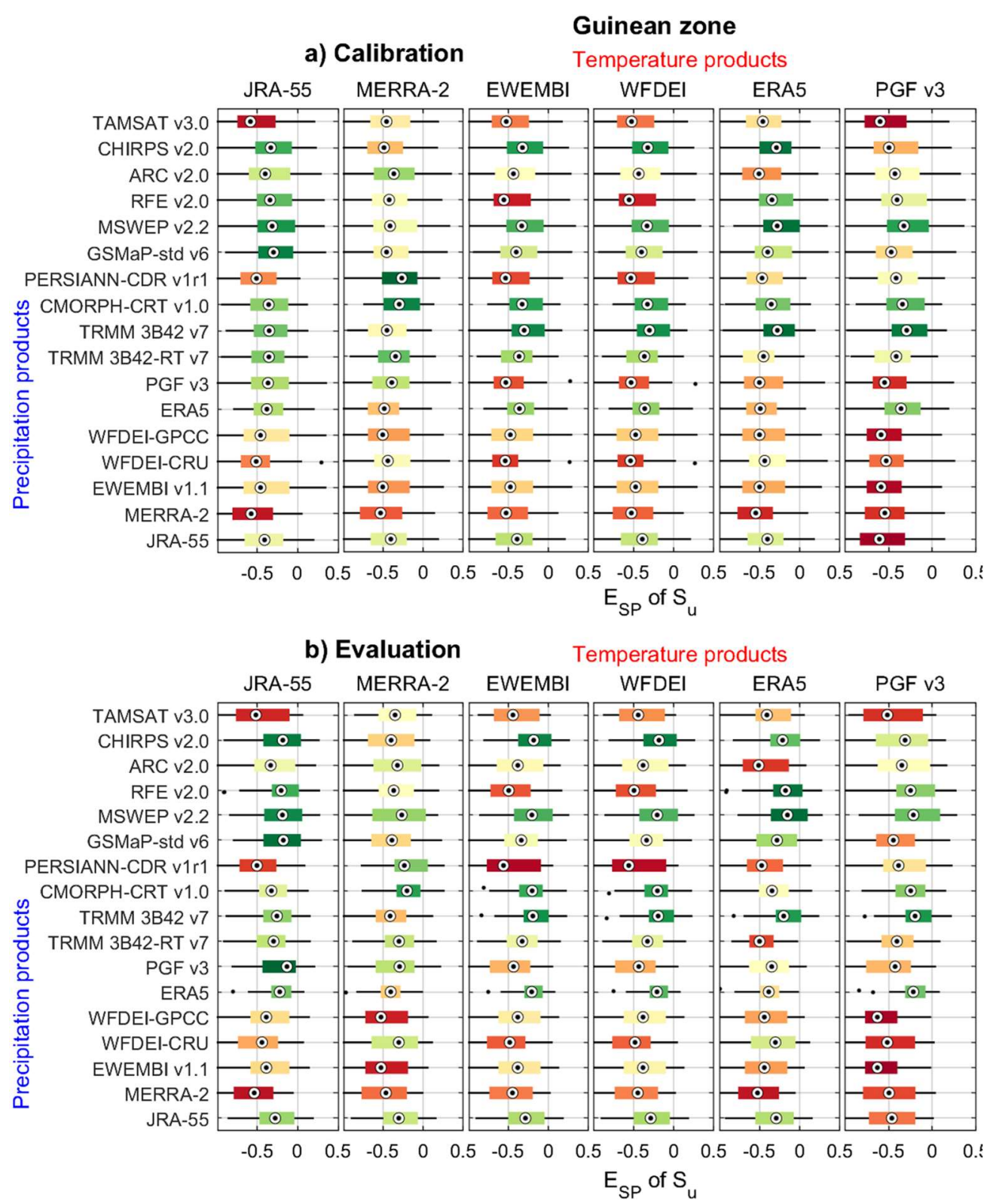

Figure S36. Spatial pattern efficiency $\left(E_{\mathrm{SP}}\right)$ of soil moisture $\left(S_{\mathrm{u}}\right)$ over (a) the calibration (2003-2008) and (b) the evaluation (2009-2012) periods for the Guinean zone, using different combinations of precipitation and temperature products for hydrological modelling. The boxplots are colored from the best (green) to the worst performance (red) based on the median value. 

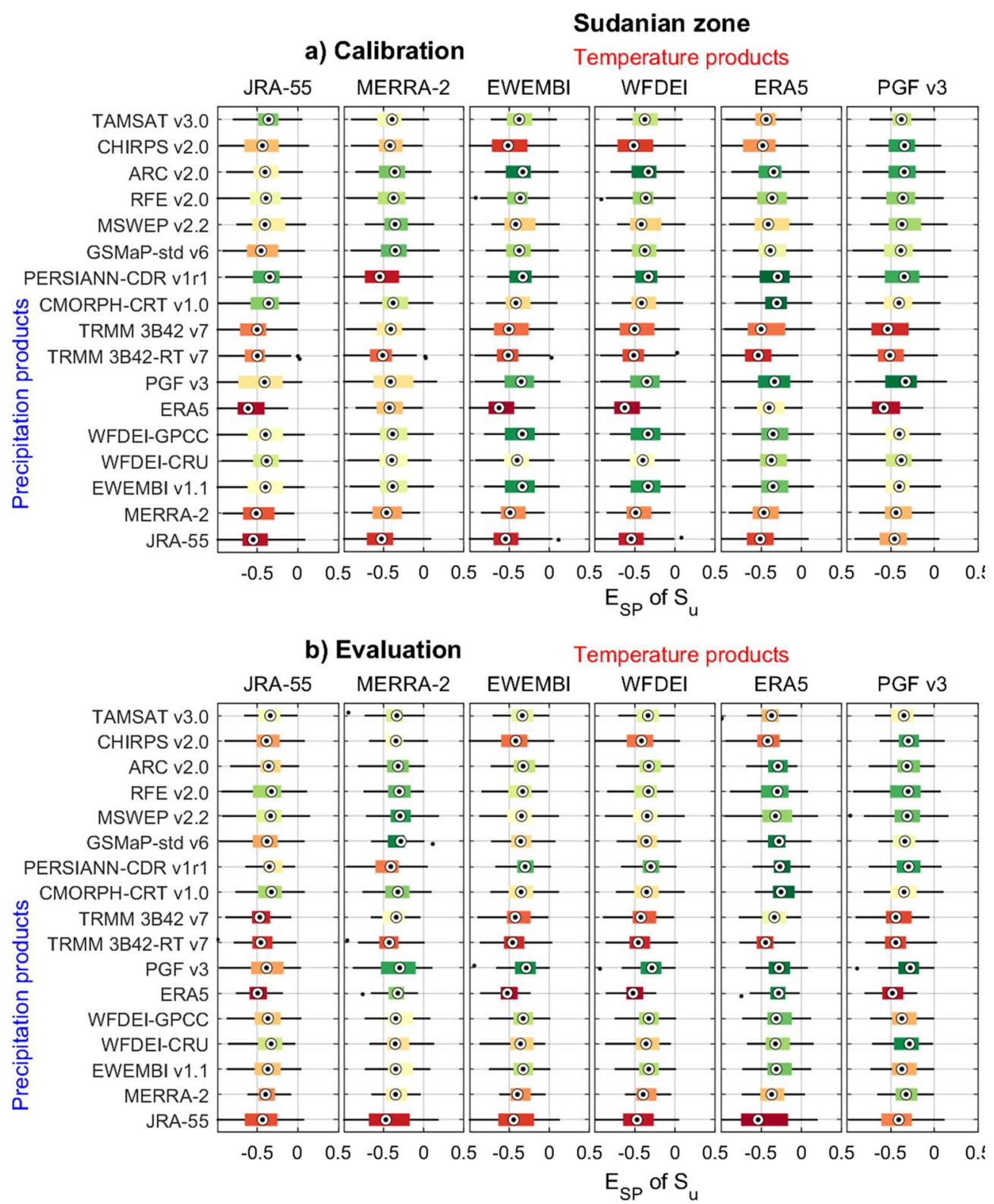

Figure S37. Spatial pattern efficiency $\left(E_{\mathrm{SP}}\right)$ of soil moisture $\left(S_{\mathrm{u}}\right)$ over (a) the calibration (2003-2008) and (b) the evaluation (2009-2012) periods for the Sudanian zone, using different combinations of precipitation and temperature products for hydrological modelling. The boxplots are colored from the best (green) to the worst performance (red) based on the median value. 

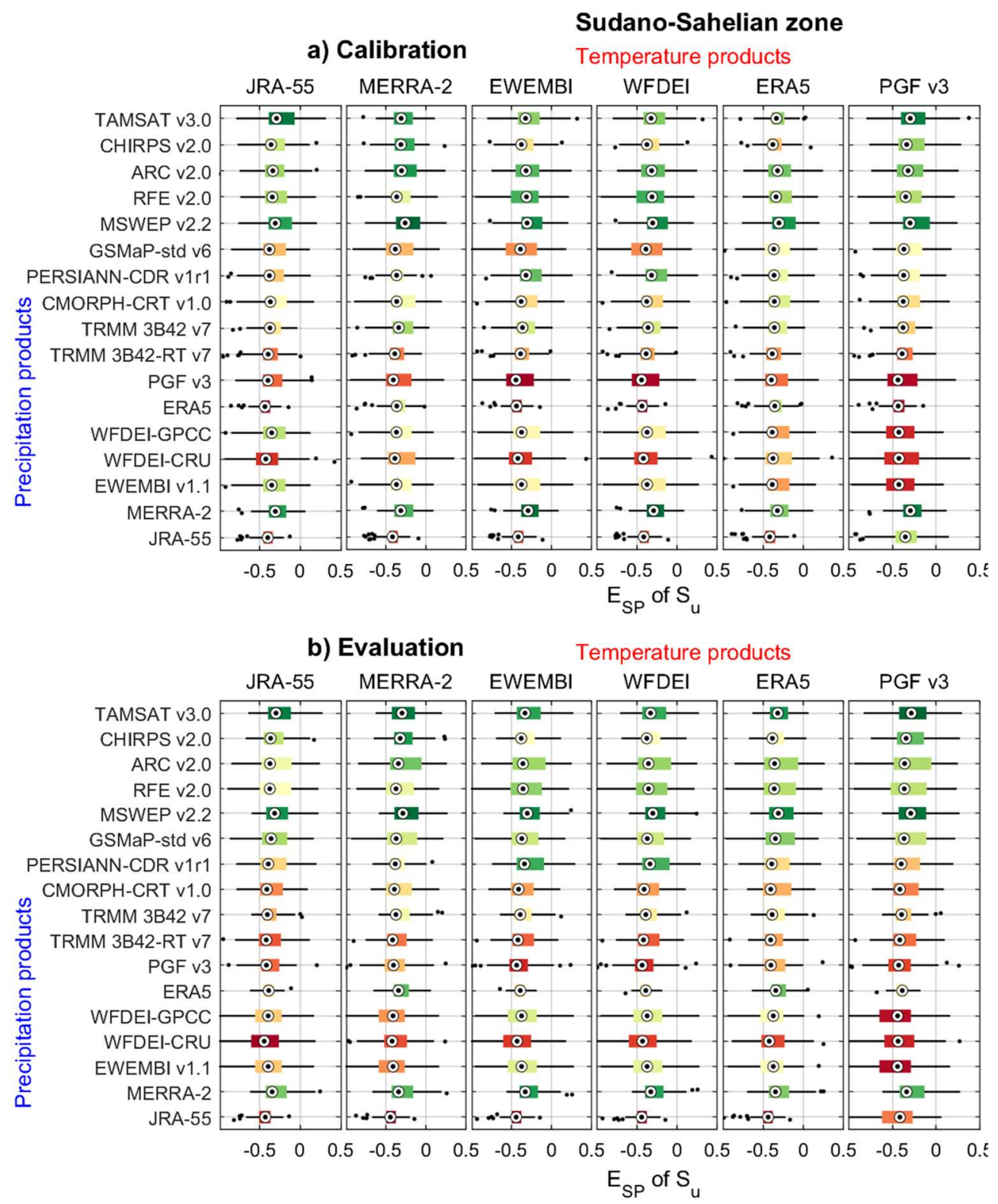

Figure S38. Spatial pattern efficiency $\left(E_{\mathrm{SP}}\right)$ of soil moisture $\left(S_{\mathrm{u}}\right)$ over (a) the calibration (2003-2008) and (b) the evaluation (2009-2012) periods for the Sudano-Sahelian zone, using different combinations of precipitation and temperature products for hydrological modelling. The boxplots are colored from the best (green) to the worst performance (red) based on the median value. 

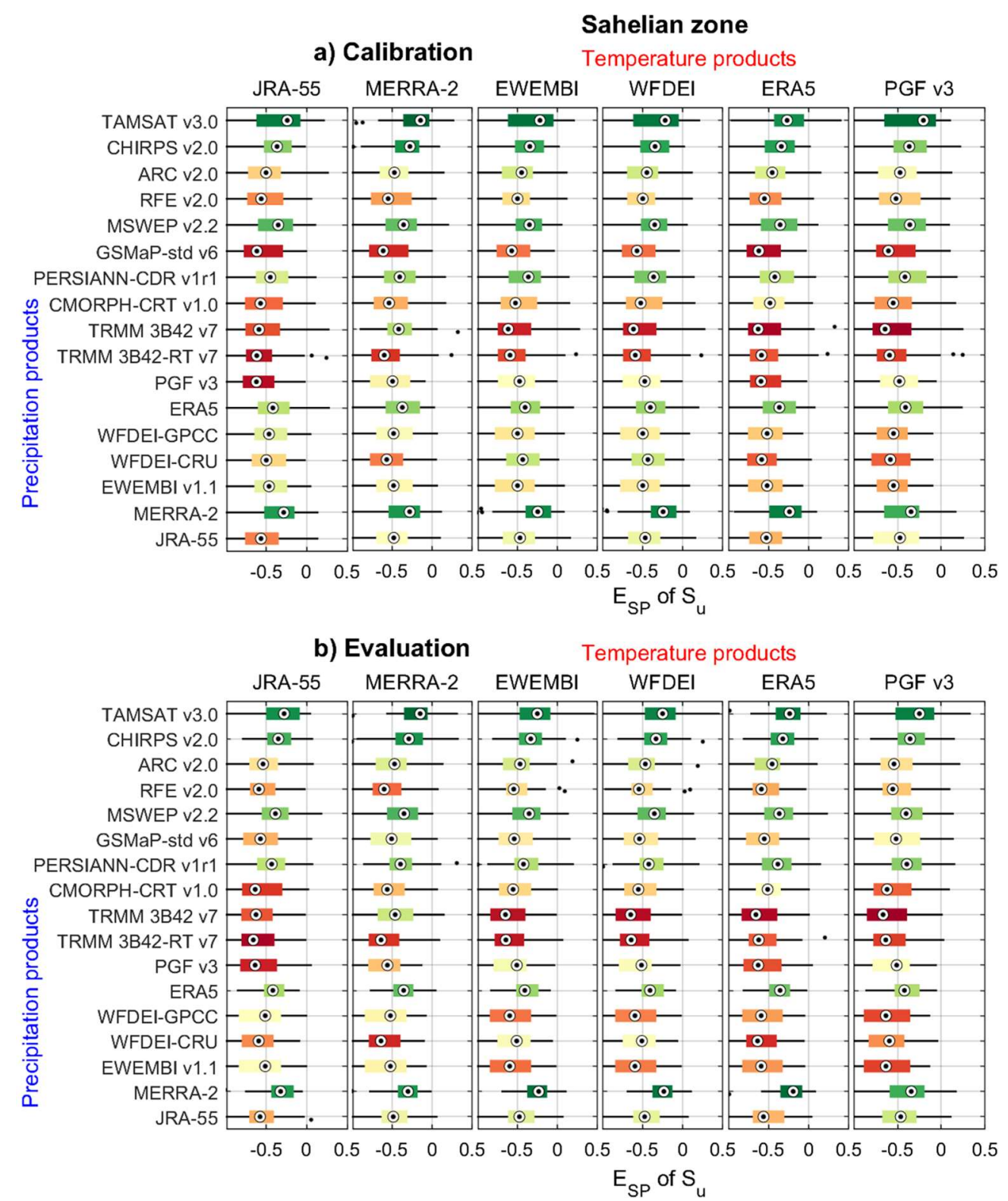

Figure S39. Spatial pattern efficiency $\left(E_{\mathrm{SP}}\right)$ of soil moisture $\left(S_{\mathrm{u}}\right)$ over (a) the calibration (2003-2008) and (b) the evaluation (2009-2012) periods for the Sahelian zone, using different combinations of precipitation and temperature products for hydrological modelling. The boxplots are colored from the best (green) to the worst performance (red) based on the median value. 
7.3 Time series

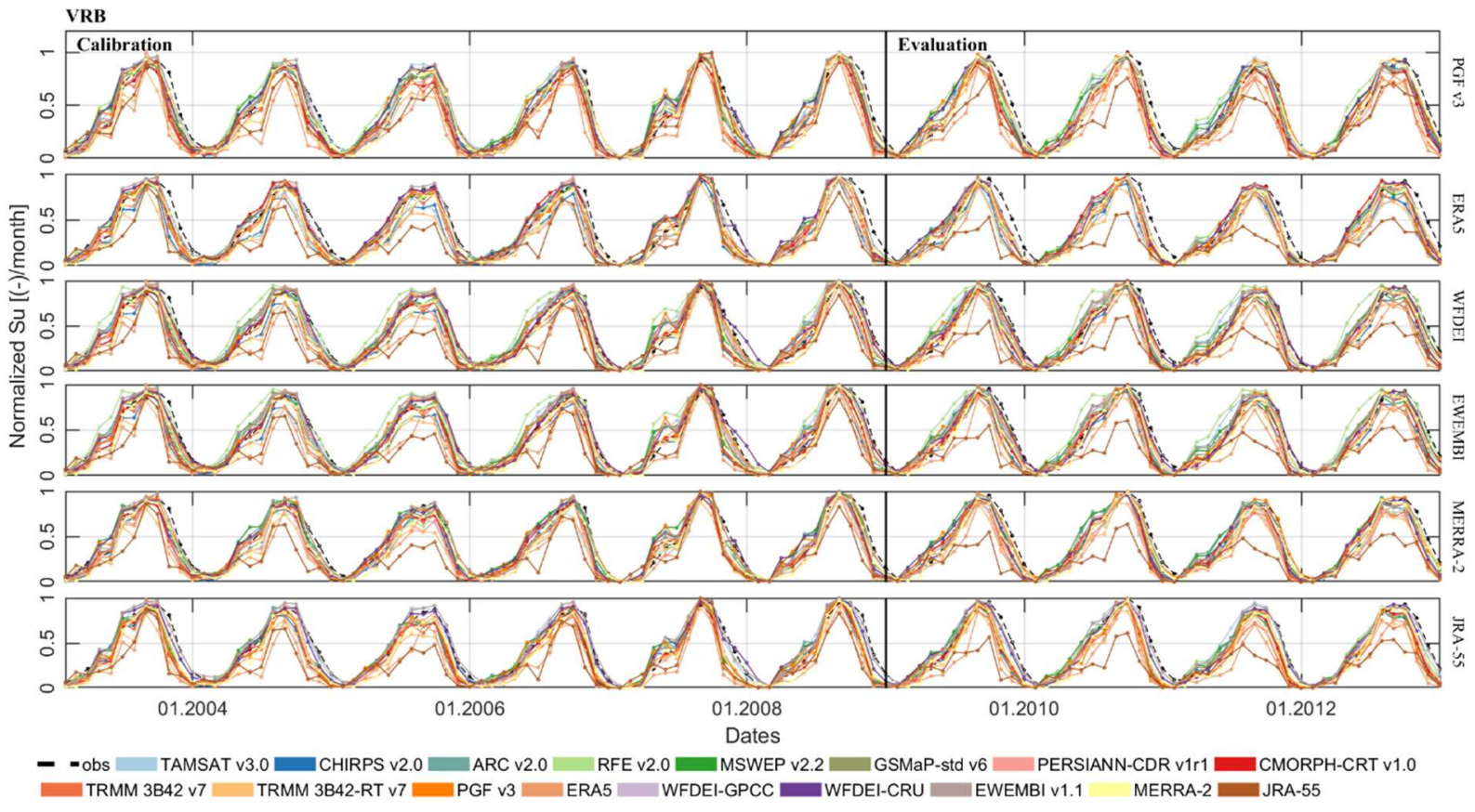

Figure S40. Monthly time series of soil moisture $\left(S_{\mathrm{u}}\right)$ in the Volta River basin (VRB) obtained for different combinations of rainfall datasets (colors in legend) and temperature datasets (right y-axis of the 6 subplots) used as inputs in a hydrological model (i.e. mHM). All values are normalized between 0 and 1.

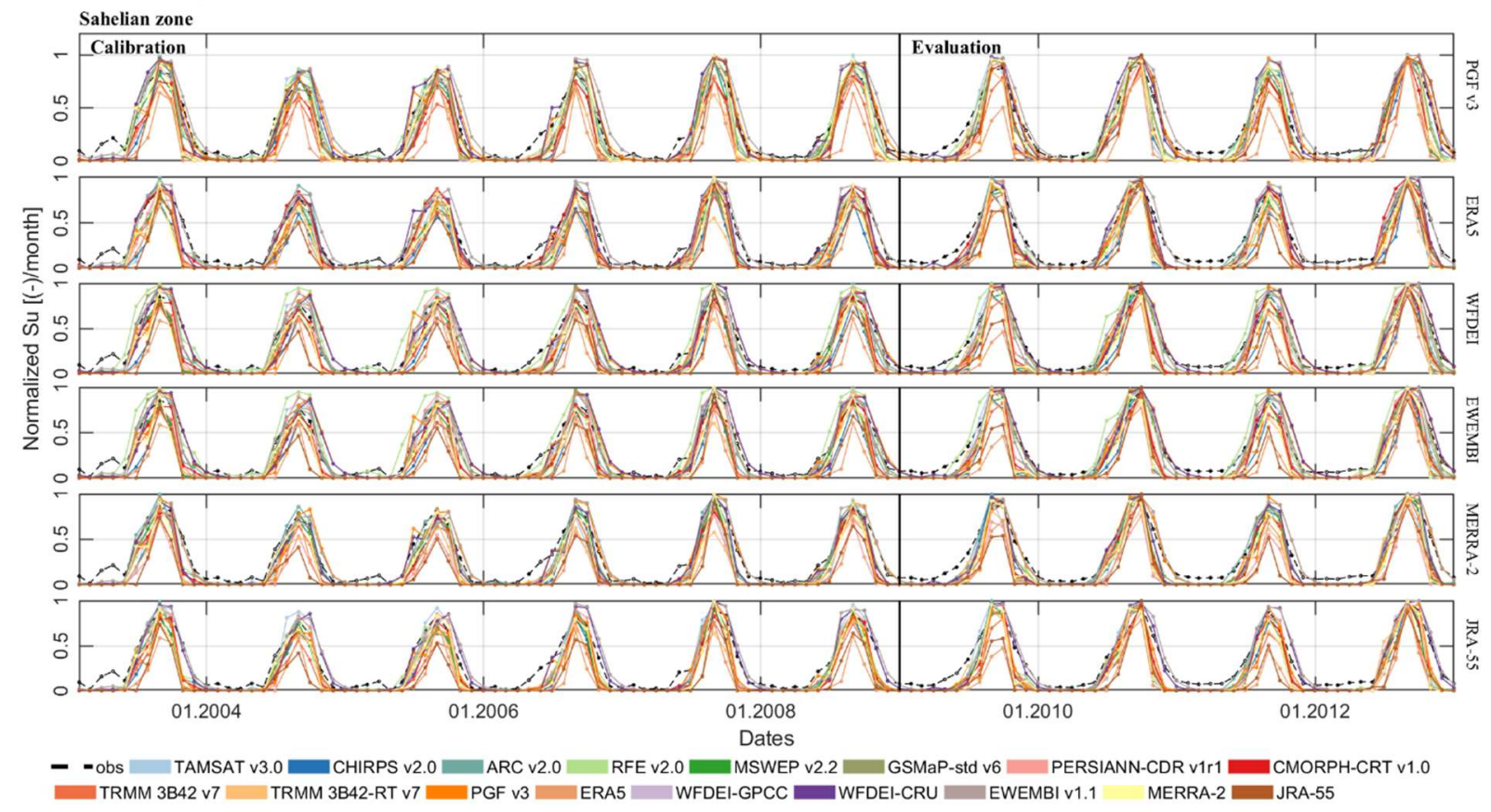

Figure S41. Monthly time series of soil moisture $\left(S_{\mathrm{u}}\right)$ in the Sahelian zone of the Volta basin obtained for different combinations of rainfall datasets (colors in legend) and temperature datasets (right y-axis of the 6 subplots) used as inputs in a hydrological model (i.e. mHM). All values are normalized between 0 and 1. 


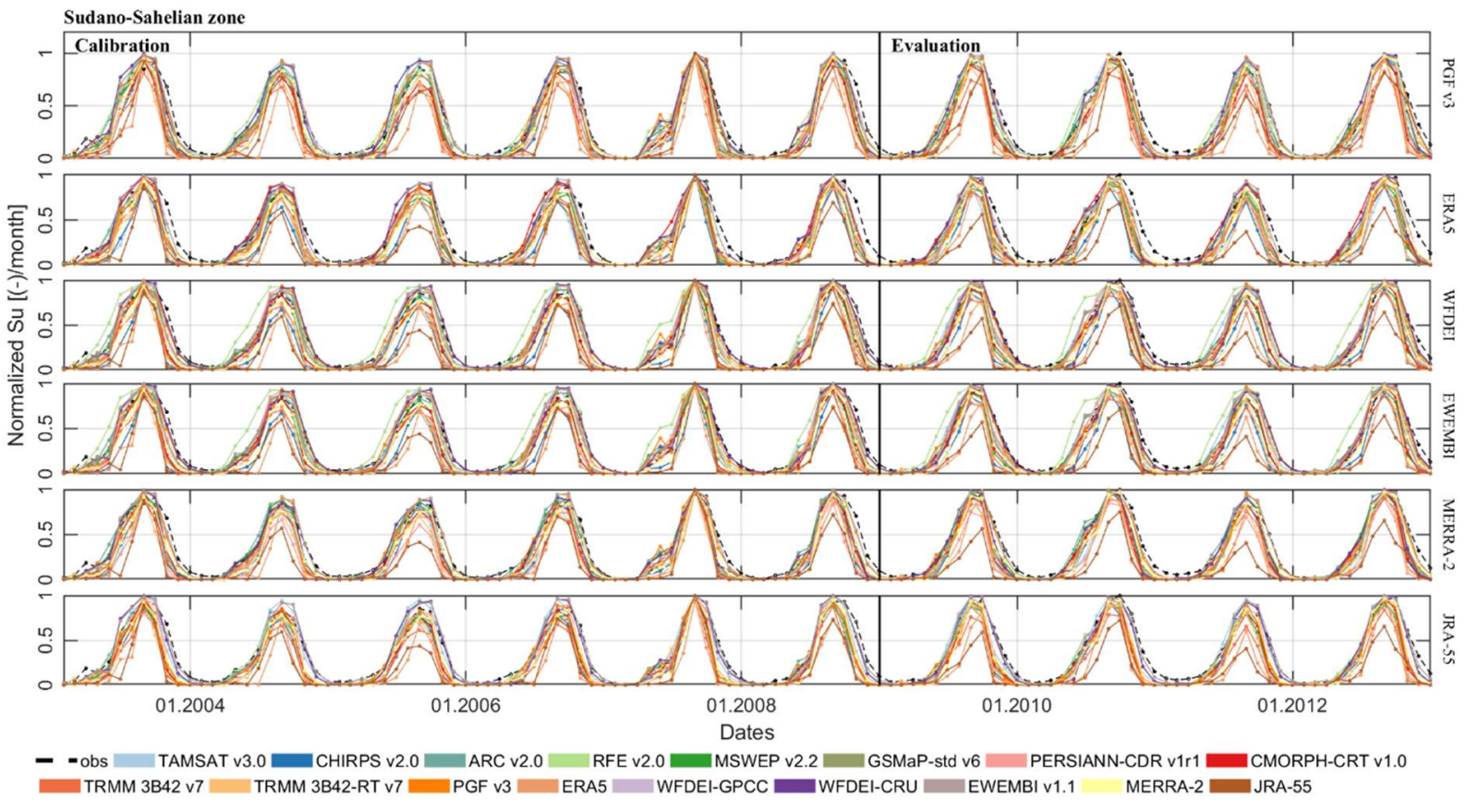

Figure S42. Monthly time series of soil moisture $\left(S_{\mathrm{u}}\right)$ in the Sudano-Sahelian zone of the Volta basin obtained for different combinations of rainfall datasets (colors in legend) and temperature datasets (right y-axis of the 6 subplots) used as inputs in a hydrological model (i.e. mHM). All values are normalized between 0 and 1.

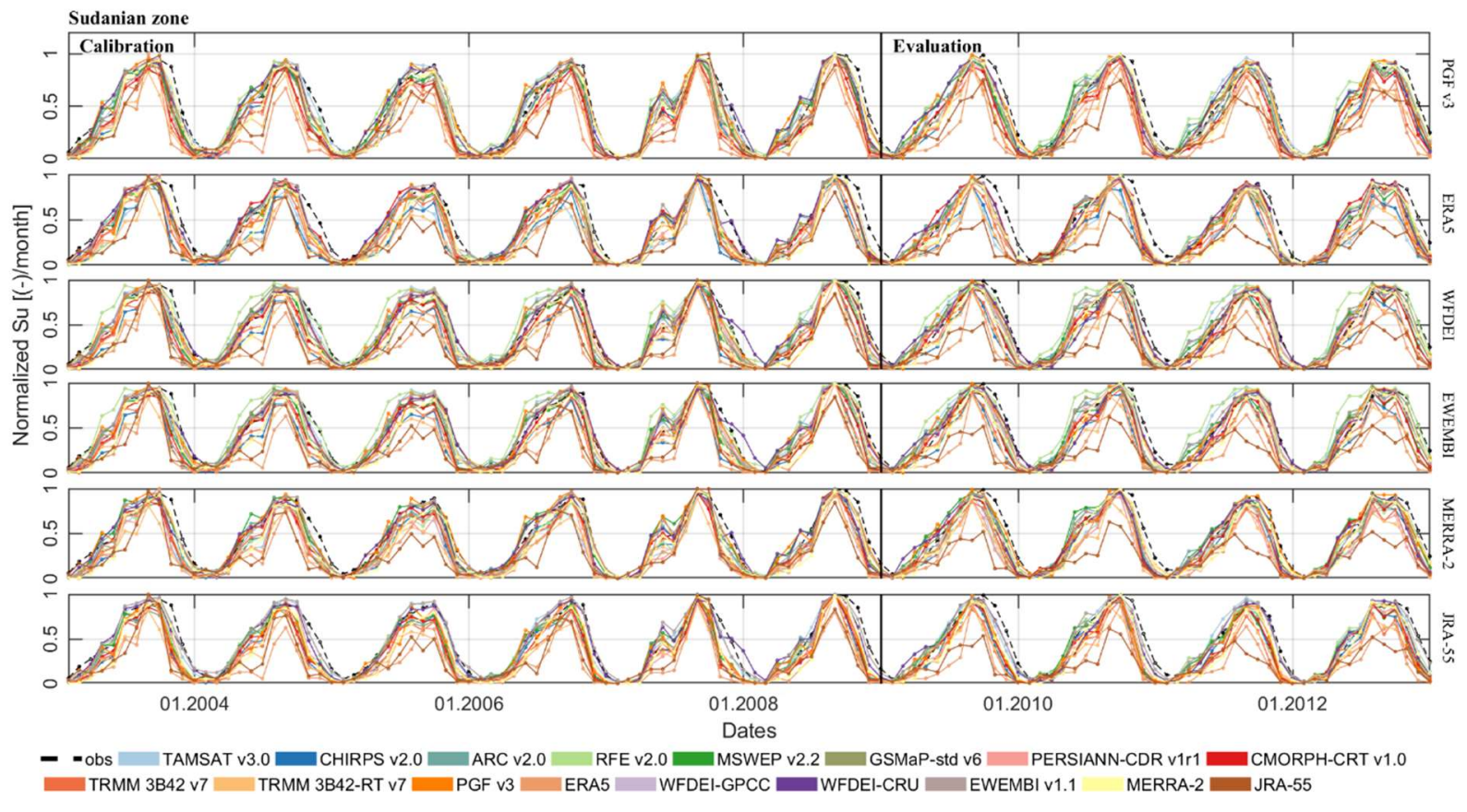

Figure S43. Monthly time series of soil moisture $\left(S_{\mathrm{u}}\right)$ in the Sudanian zone of the Volta basin obtained for different combinations of rainfall datasets (colors in legend) and temperature datasets (right y-axis of the 6 subplots) used as inputs in a hydrological model (i.e. mHM). All values are normalized between 0 and 1 . 


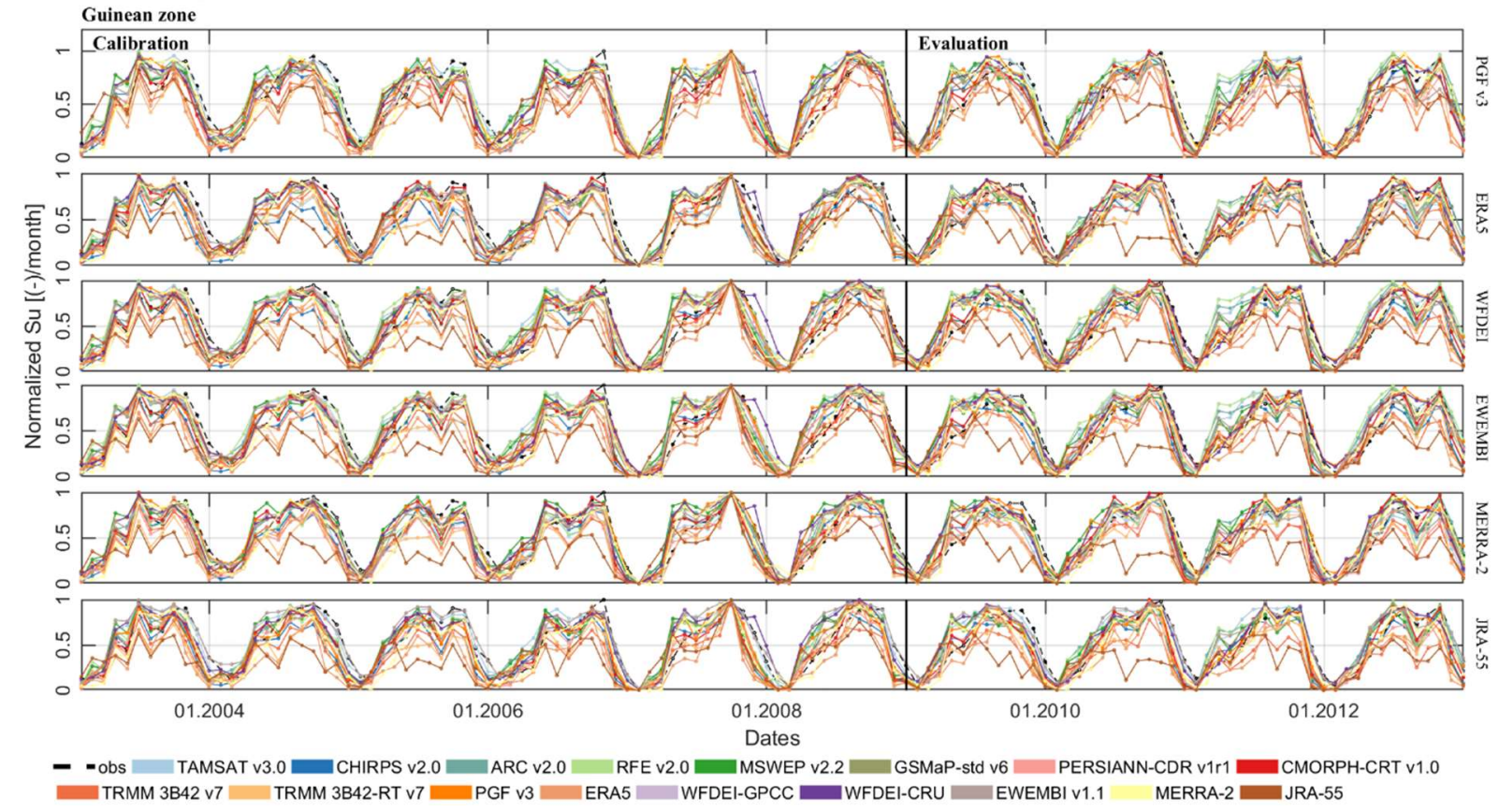

Figure S44. Monthly time series of soil moisture $\left(S_{\mathrm{u}}\right)$ in the Guinean zone of the Volta basin obtained for different combinations of rainfall datasets (colors in legend) and temperature datasets (right y-axis of the 6 subplots) used as inputs in a hydrological model (i.e. mHM). All values are normalized between 0 and 1 . 
7.4 Maps of long-term average

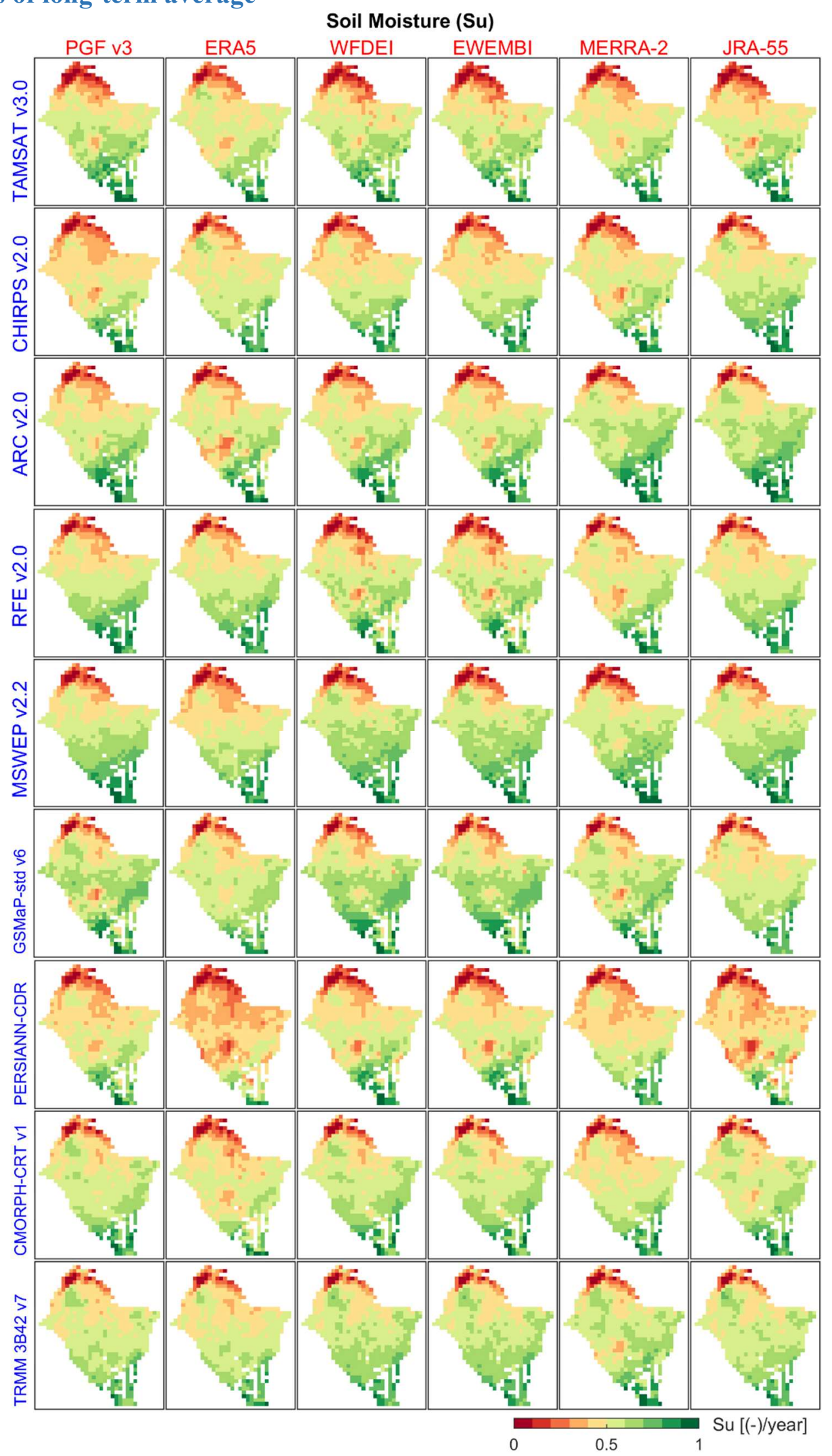

Figure S45. (Part1/2) Maps of long-term (2003-2012) annual average of soil moisture $\left(S_{\mathrm{u}}\right)$ obtained as outputs of hydrological modelling using different combinations of rainfall datasets (y-axis, blue font) and temperature datasets (x-axis, red font). The values are normalized for better emphasizing on patterns and using a unique color scale. 


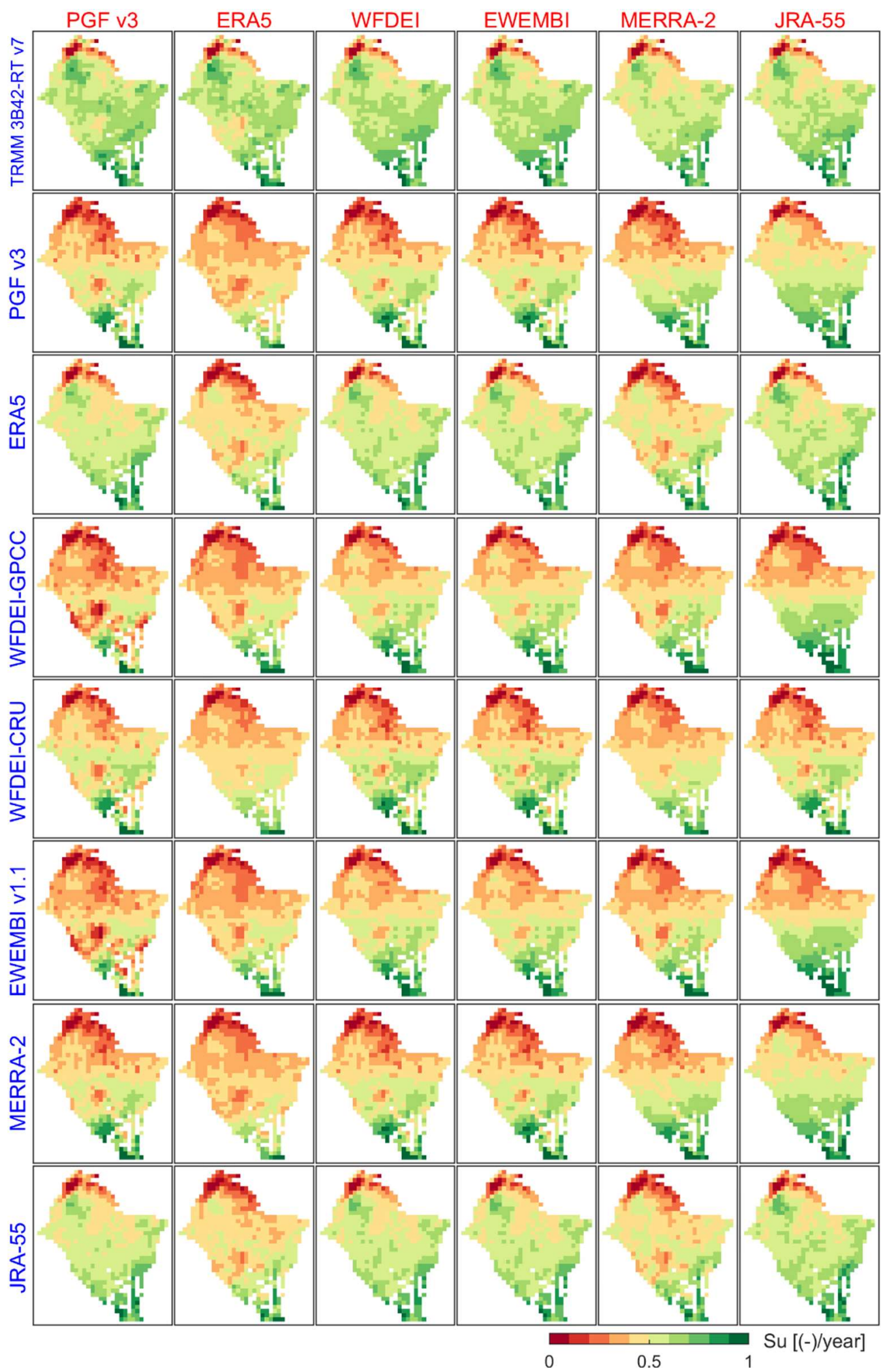

Figure S46. (Part2/2) Maps of long-term (2003-2012) annual average of soil moisture $\left(S_{\mathrm{u}}\right)$ obtained as outputs of hydrological modelling using different combinations of rainfall datasets ( $\mathrm{y}$-axis, blue font) and temperature datasets (x-axis, red font). The values are normalized for better emphasizing on patterns and using a unique color scale. 


\section{Model performance for evaporation}

\subsection{Ranking of rainfall and temperature datasets}

Table S16. Ranking of rainfall and temperature datasets based on the Pearson correlation coefficient $(r)$ of actual evaporation $\left(E_{\mathrm{a}}\right)$ over the simulation period. The mean and the second-order coefficient of variation $\left(V_{2}\right)$ are calculated across 6 temperature datasets for each rainfall dataset, and across 17 rainfall datasets for each temperature dataset. The rank is given from the best (green) to the worst performance (red) for 4 climatic zones in the Volta River basin (VRB)

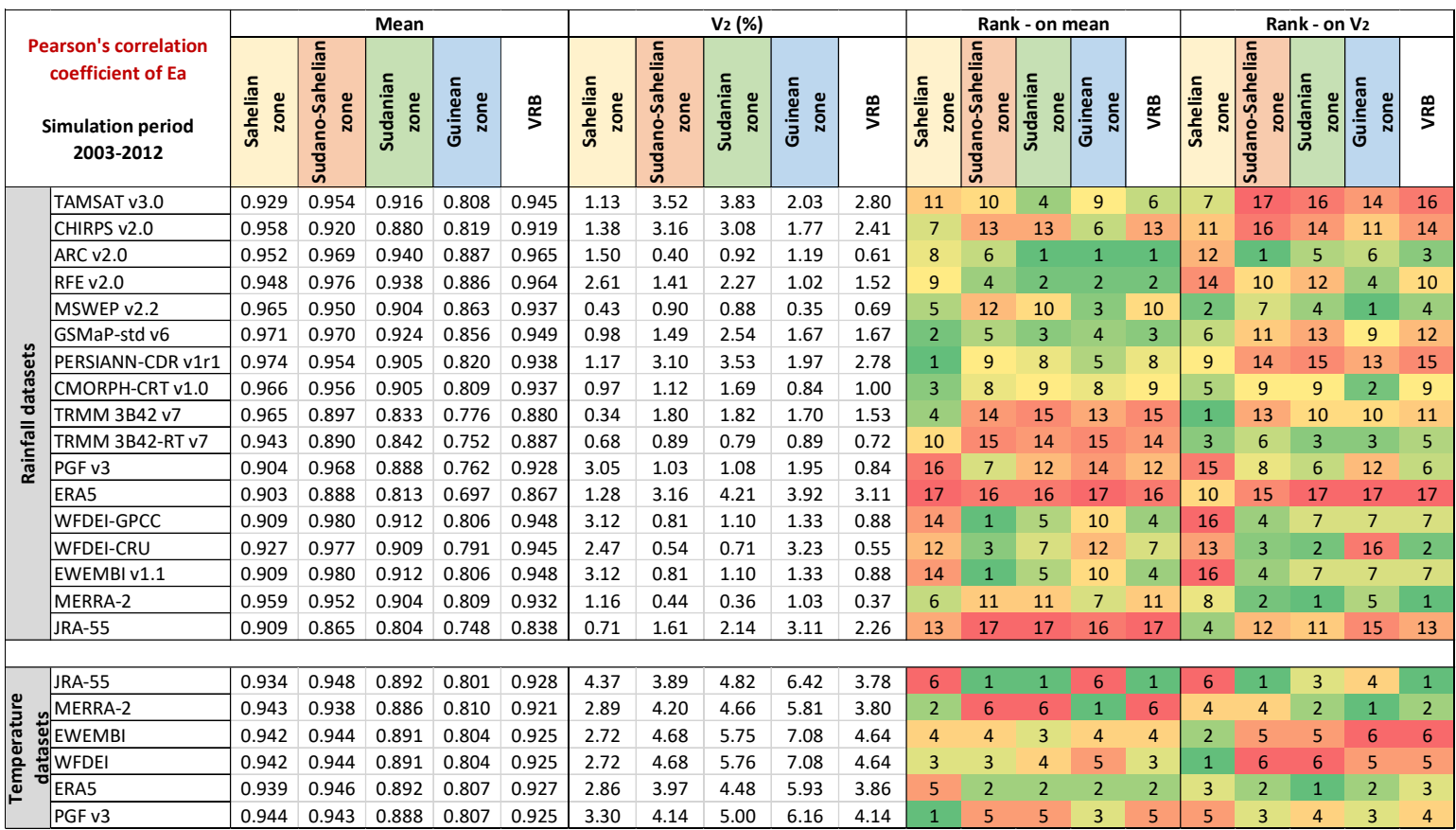

Table S17. Ranking of rainfall and temperature datasets based on the spatial pattern efficiency $\left(E_{\mathrm{SP}}\right)$ of actual evaporation $\left(E_{\mathrm{a}}\right)$ over the simulation period. The mean and the second-order coefficient of variation $\left(V_{2}\right)$ are calculated across 6 temperature datasets for each rainfall dataset, and across 17 rainfall datasets for each temperature dataset. The rank is given from the best (green) to the worst performance (red) for 4 climatic zones in the Volta River basin (VRB)

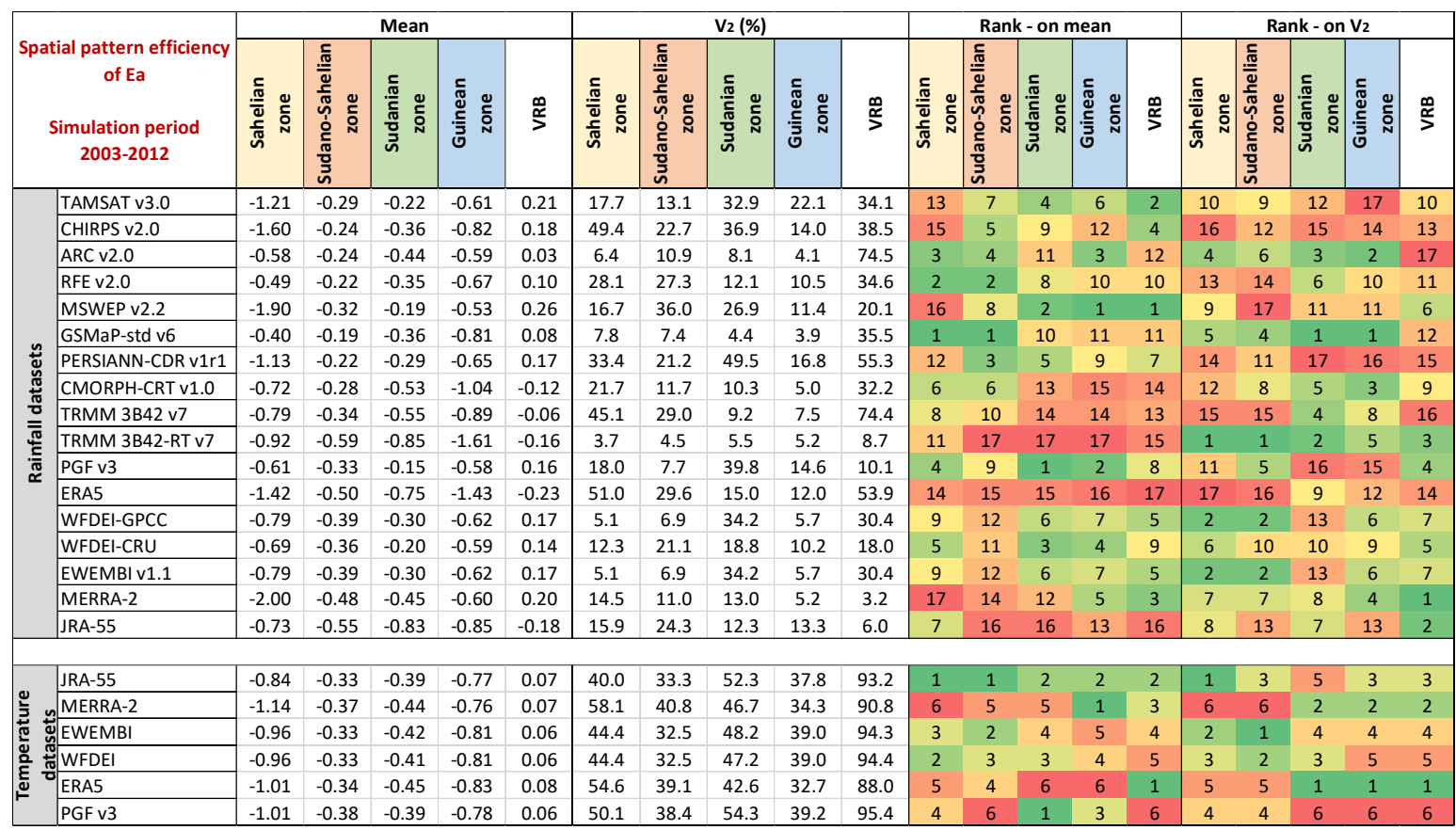




\subsection{Boxplots}

8.2.1 Combined calibration and evaluation periods

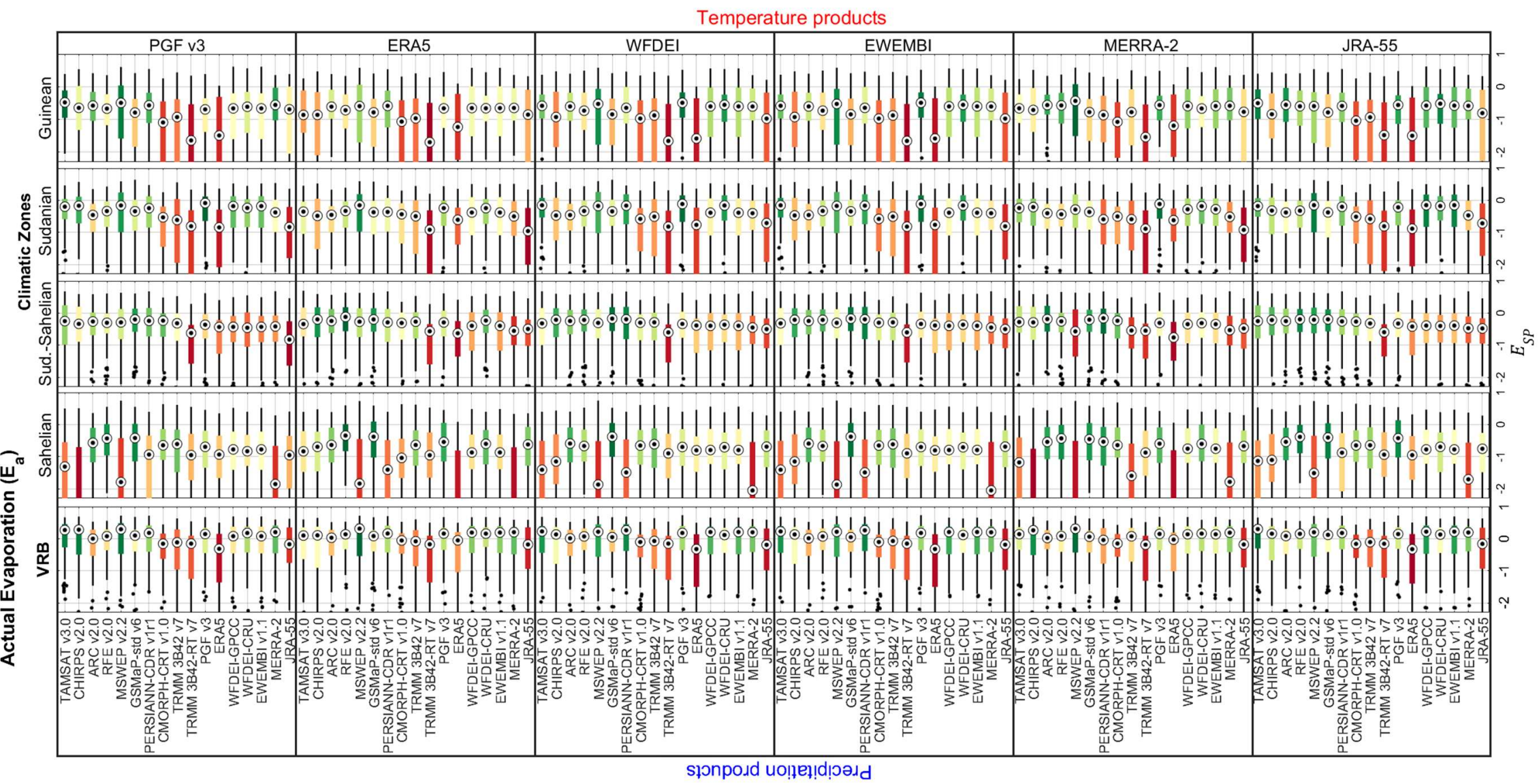

Figure S47. Spatial pattern efficiency $\left(E_{\mathrm{SP}}\right)$ of actual evaporation $\left(E_{\mathrm{a}}\right)$ over the entire simulation period (2003-2012) for the Volta River basin (VRB) and its climatic zones, using different combinations of precipitation and temperature products for hydrological modelling. The boxplots are colored from the best (green) to the worst performance (red) based on the median value. 


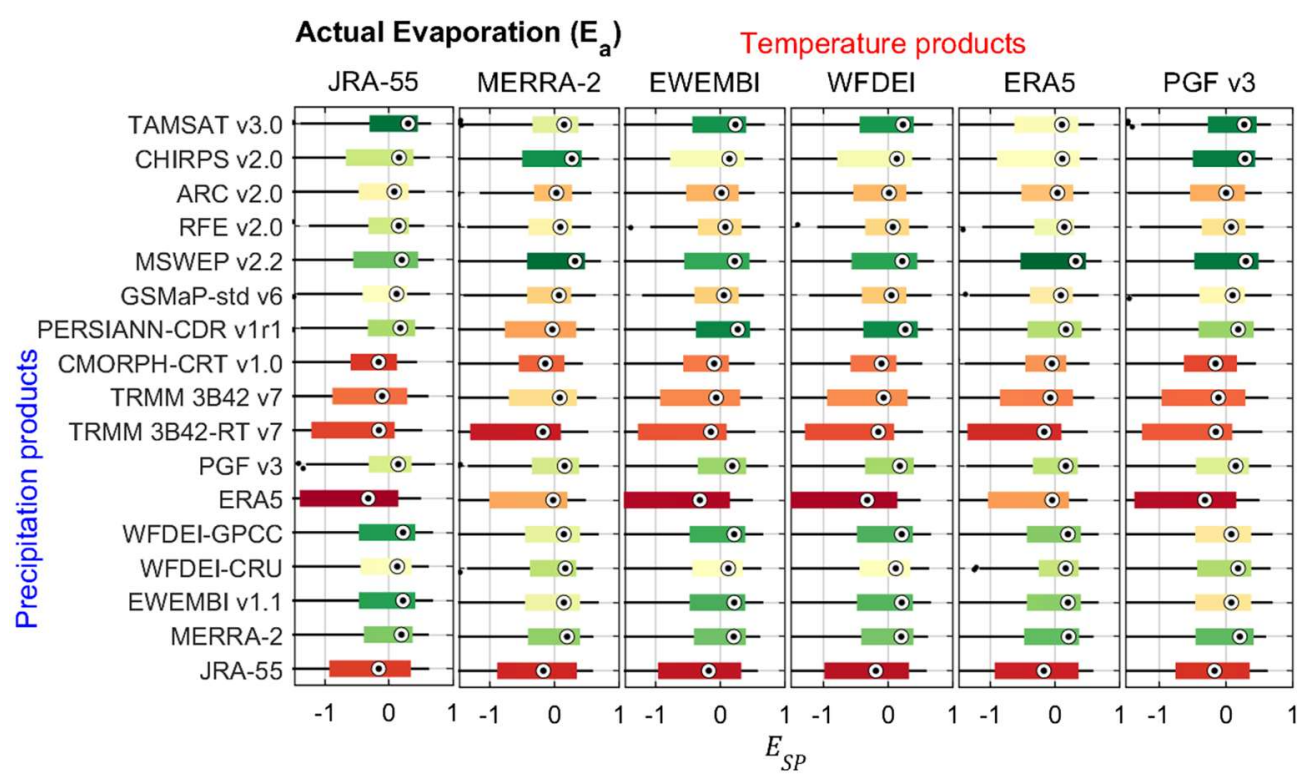

Figure S48. Spatial pattern efficiency $\left(E_{\mathrm{SP}}\right)$ of actual evaporation $\left(E_{\mathrm{a}}\right)$ over the entire simulation period (20032012) for the Volta River basin (VRB), using different combinations of precipitation and temperature products for hydrological modelling. The boxplots are colored from the best (green) to the worst performance (red) based on the median value.

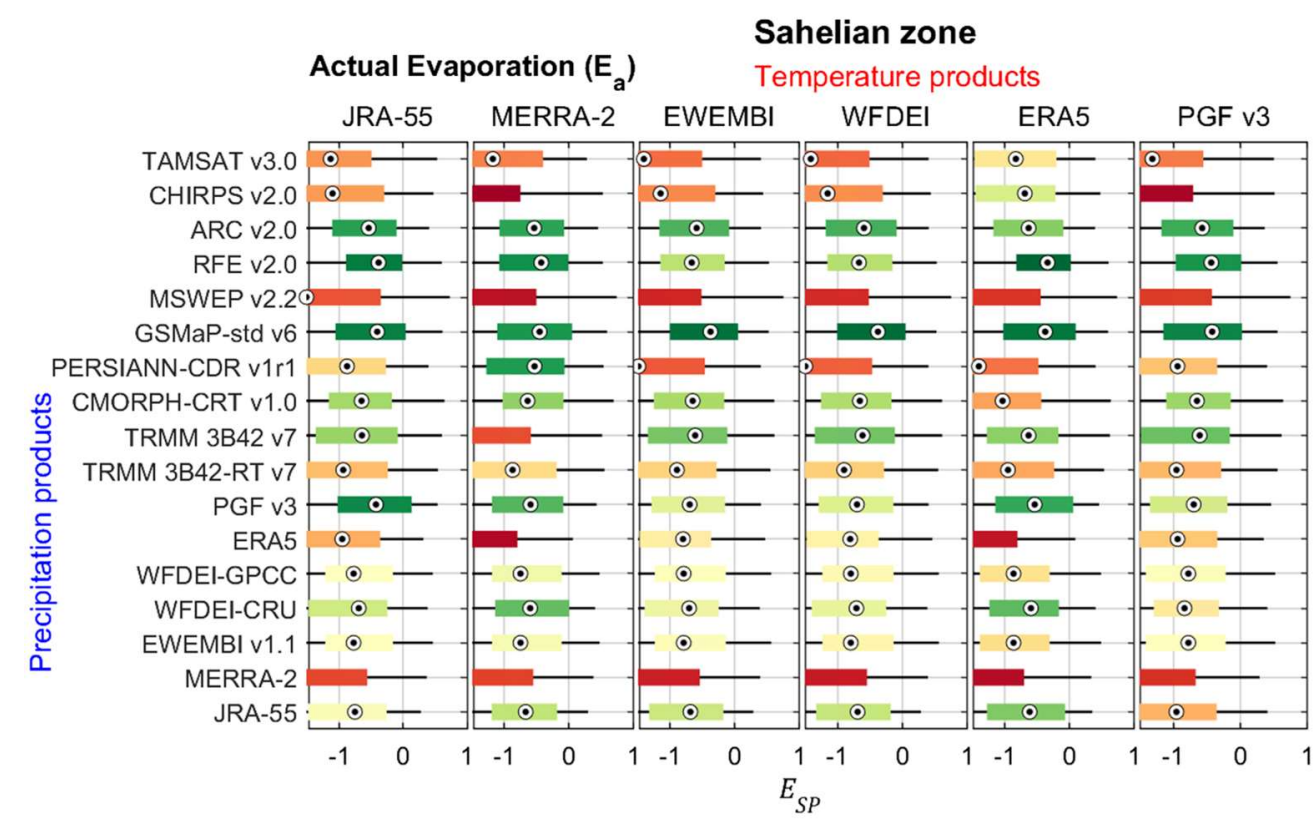

Figure S49. Spatial pattern efficiency $\left(E_{\mathrm{SP}}\right)$ of actual evaporation $\left(E_{\mathrm{a}}\right)$ over the entire simulation period (20032012) for the Sahelian zone, using different combinations of precipitation and temperature products for hydrological modelling. The boxplots are colored from the best (green) to the worst performance (red) based on the median value. 


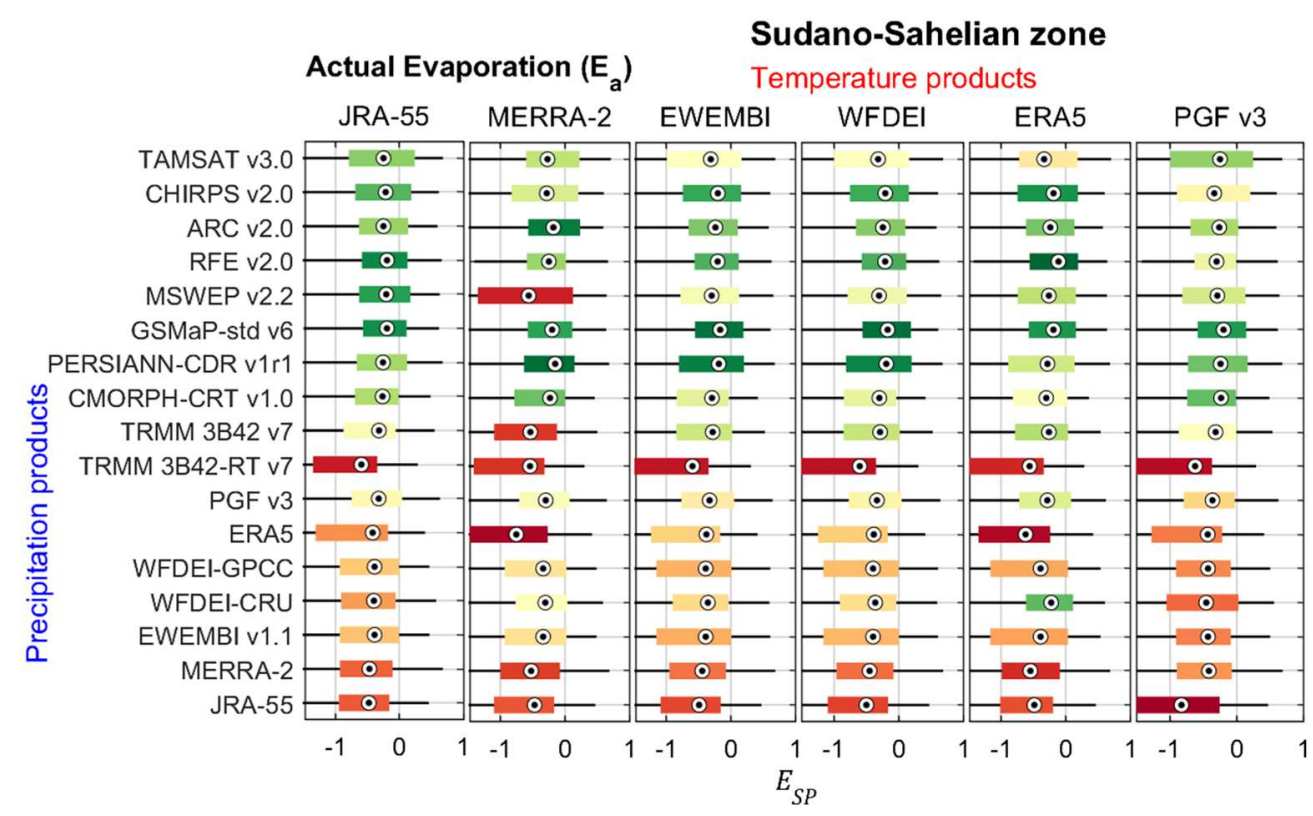

Figure S50. Spatial pattern efficiency $\left(E_{\mathrm{SP}}\right)$ of actual evaporation $\left(E_{\mathrm{a}}\right)$ over the entire simulation period (20032012) for the Sudano-Sahelian zone, using different combinations of precipitation and temperature products for hydrological modelling. The boxplots are colored from the best (green) to the worst performance (red) based on the median value.

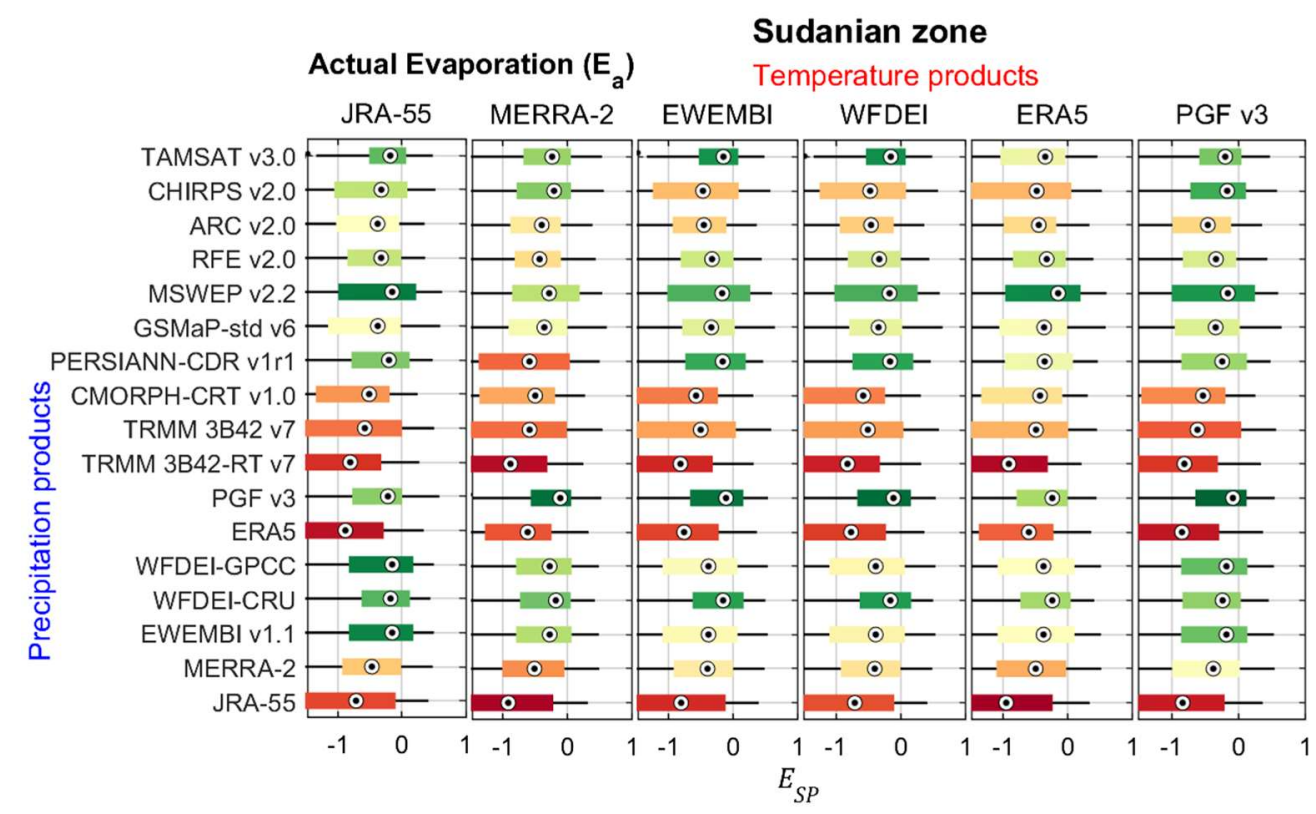

Figure S51. Spatial pattern efficiency $\left(E_{\mathrm{SP}}\right)$ of actual evaporation $\left(E_{\mathrm{a}}\right)$ over the entire simulation period (20032012) for the Sudanian zone, using different combinations of precipitation and temperature products for hydrological modelling. The boxplots are colored from the best (green) to the worst performance (red) based on the median value. 


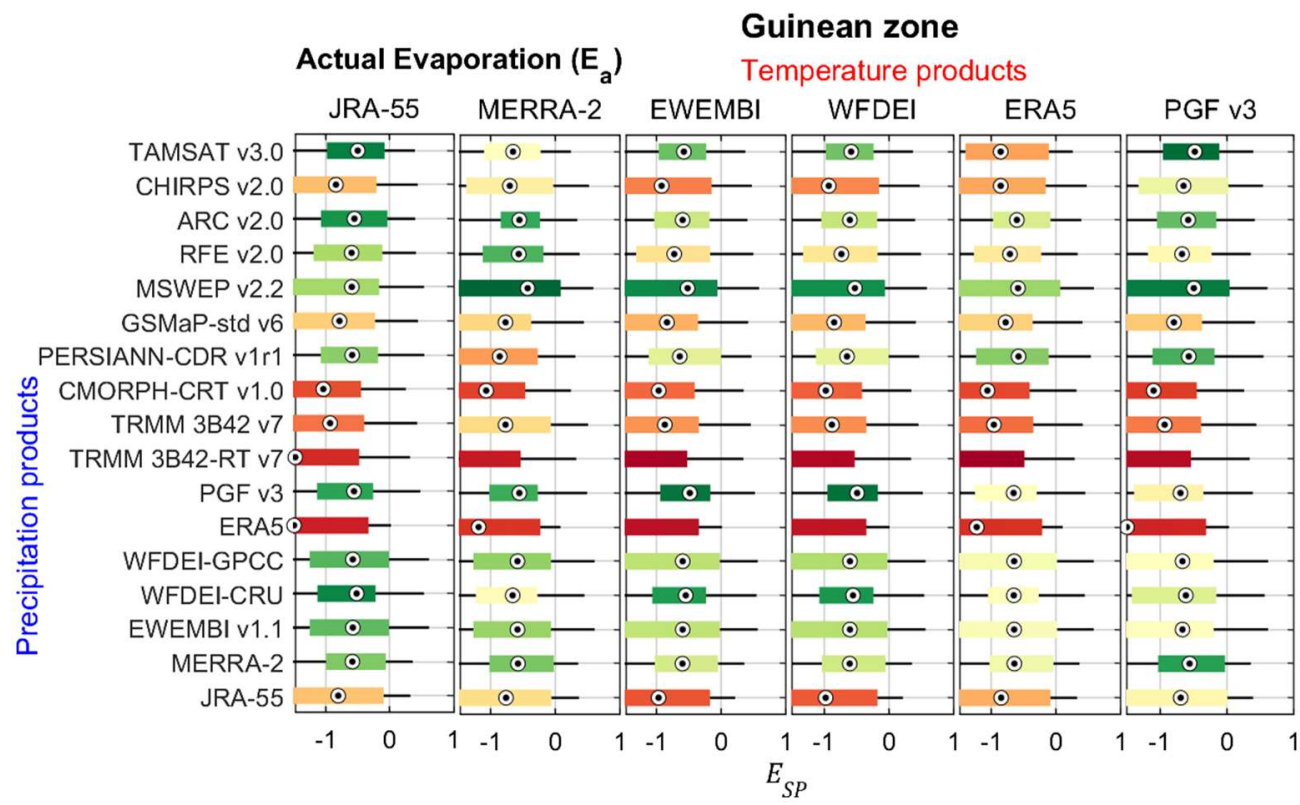

Figure S52. Spatial pattern efficiency $\left(E_{\mathrm{SP}}\right)$ of actual evaporation $\left(E_{\mathrm{a}}\right)$ over the entire simulation period (20032012) for the Guinean zone, using different combinations of precipitation and temperature products for hydrological modelling. The boxplots are colored from the best (green) to the worst performance (red) based on the median value. 


\subsubsection{Separated calibration and evaluation periods}
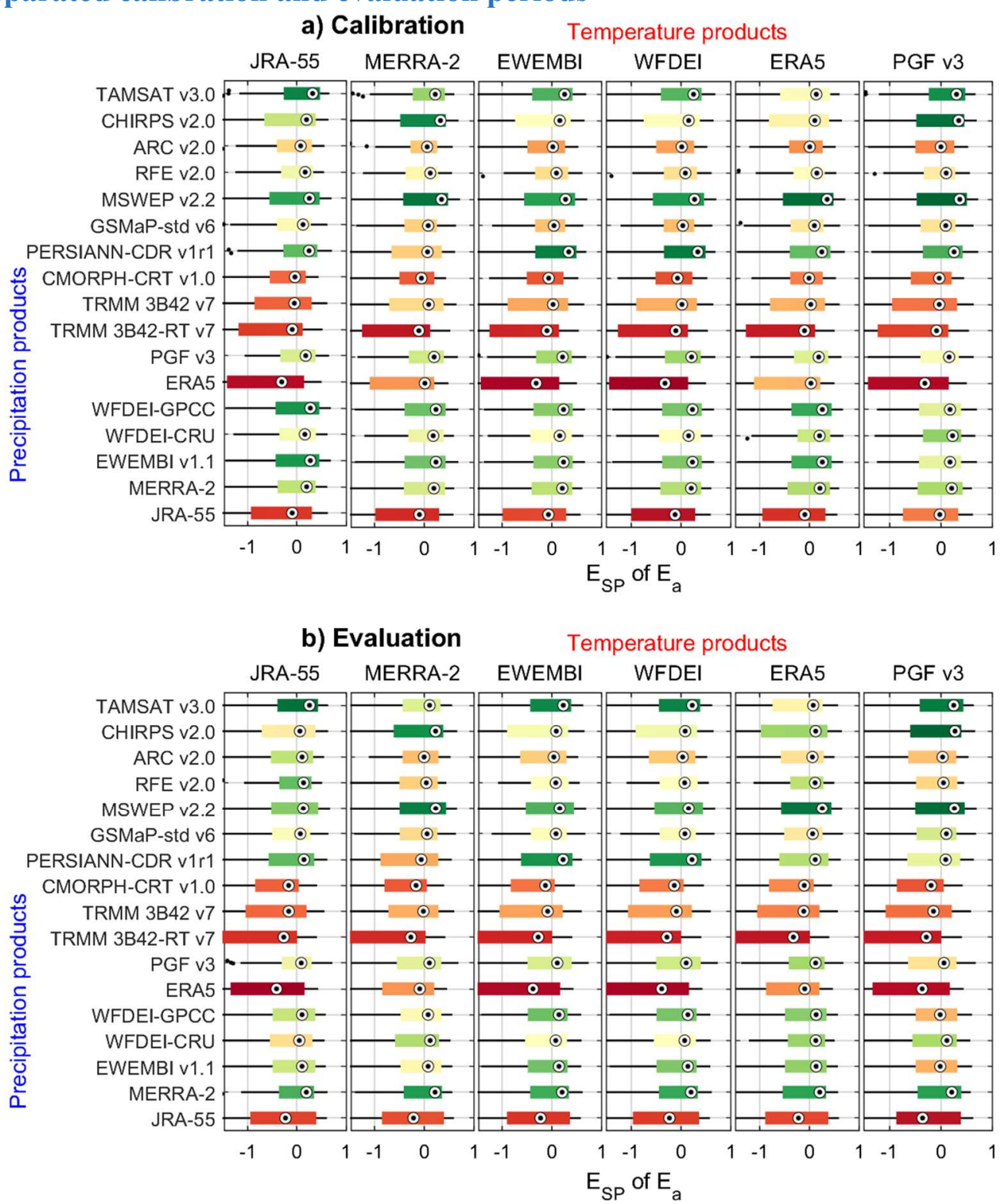

Figure S53. Spatial pattern efficiency $\left(E_{\mathrm{SP}}\right)$ of actual evaporation $\left(E_{\mathrm{a}}\right)$ over (a) the calibration (2003-2008) and (b) the evaluation (2009-2012) periods for the Volta River basin (VRB), using different combinations of precipitation and temperature products for hydrological modelling. The boxplots are colored from the best (green) to the worst performance (red) based on the median value. 

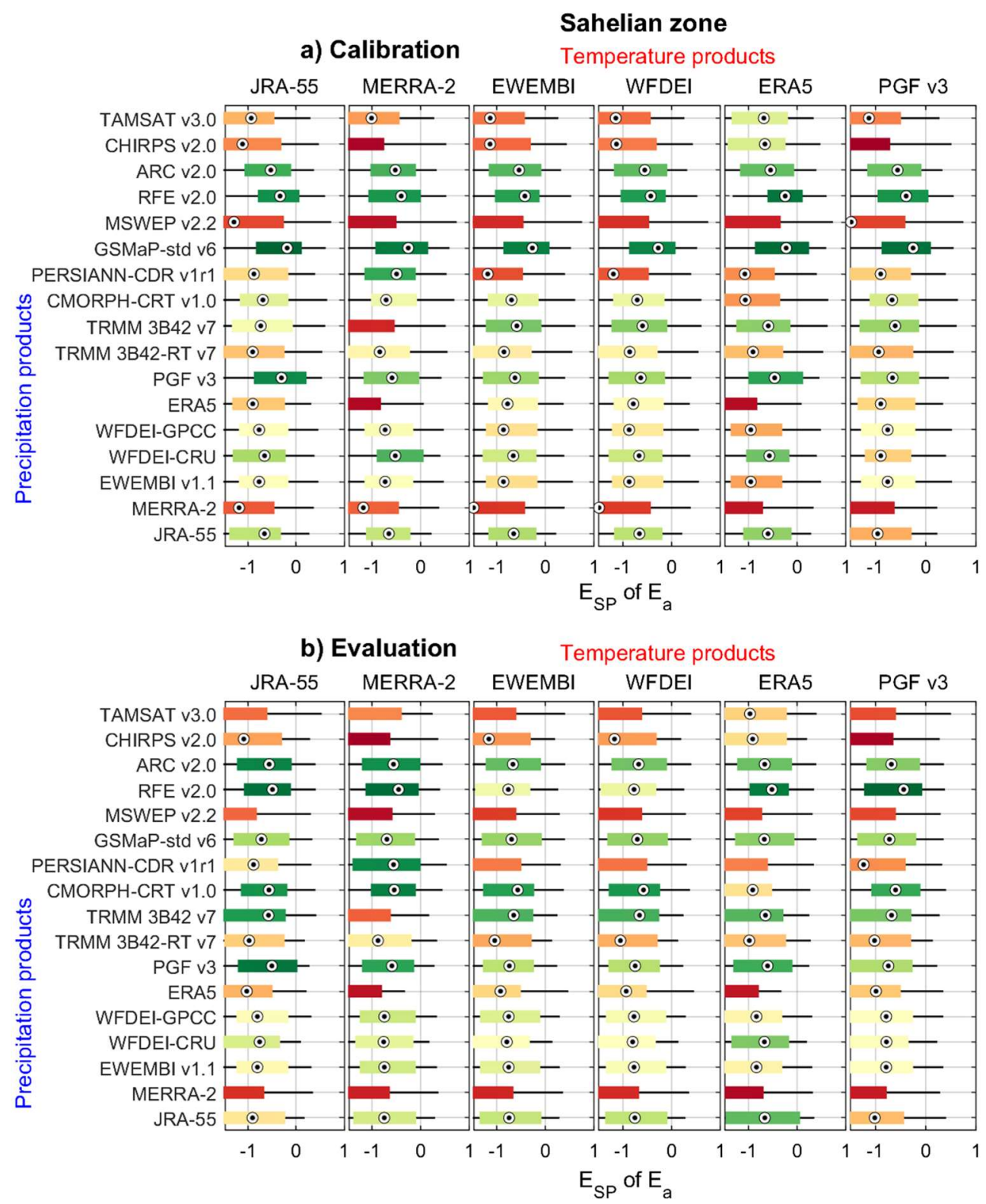

Figure S54. Spatial pattern efficiency $\left(E_{\mathrm{SP}}\right)$ of actual evaporation $\left(E_{\mathrm{a}}\right)$ over (a) the calibration (2003-2008) and (b) the evaluation (2009-2012) periods for the Sahelian zone, using different combinations of precipitation and temperature products for hydrological modelling. The boxplots are colored from the best (green) to the worst performance (red) based on the median value. 

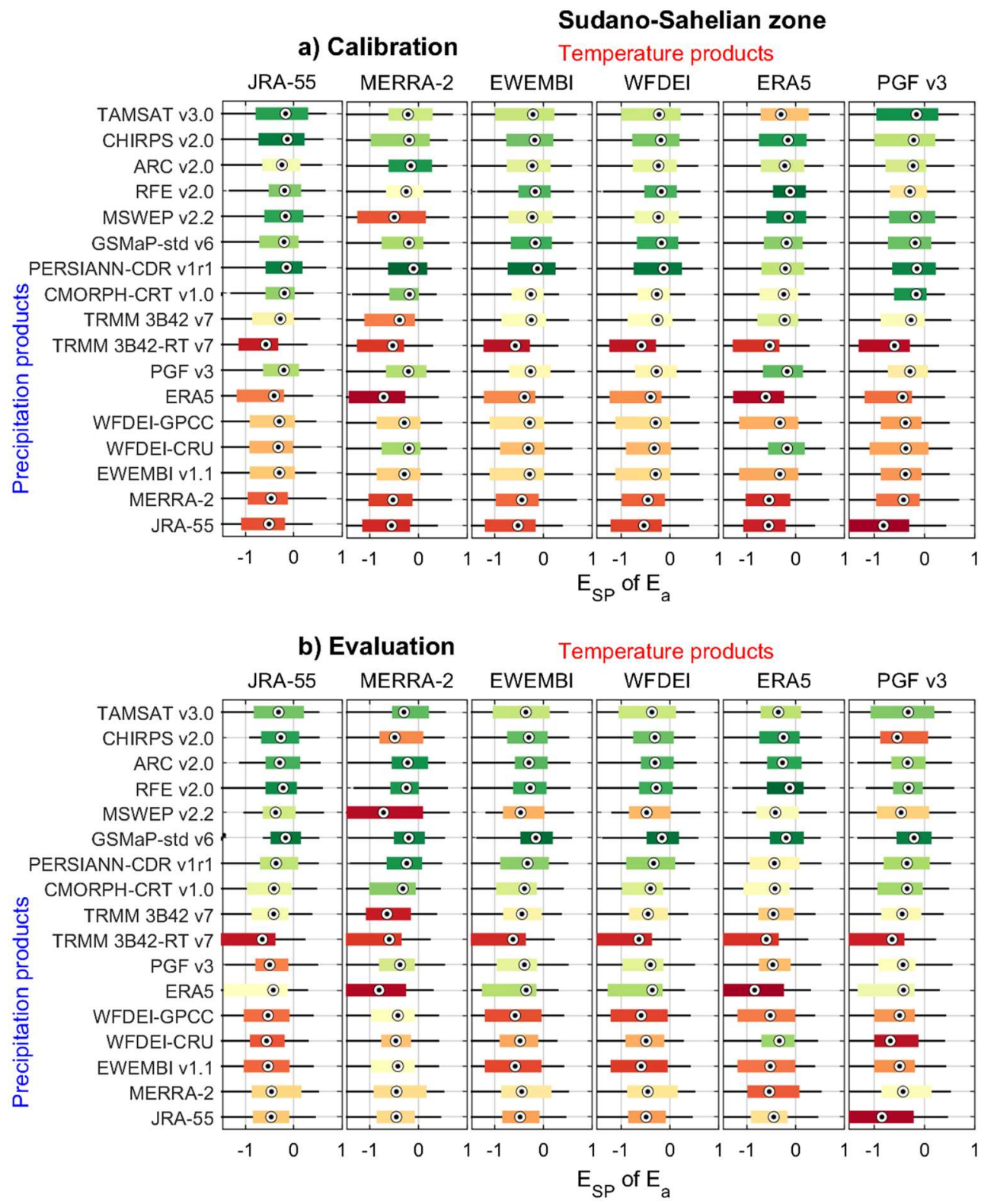

Figure S55. Spatial pattern efficiency $\left(E_{\mathrm{SP}}\right)$ of actual evaporation $\left(E_{\mathrm{a}}\right)$ over (a) the calibration (2003-2008) and (b) the evaluation (2009-2012) periods for the Sudano-Sahelian zone, using different combinations of precipitation and temperature products for hydrological modelling. The boxplots are colored from the best (green) to the worst performance (red) based on the median value. 

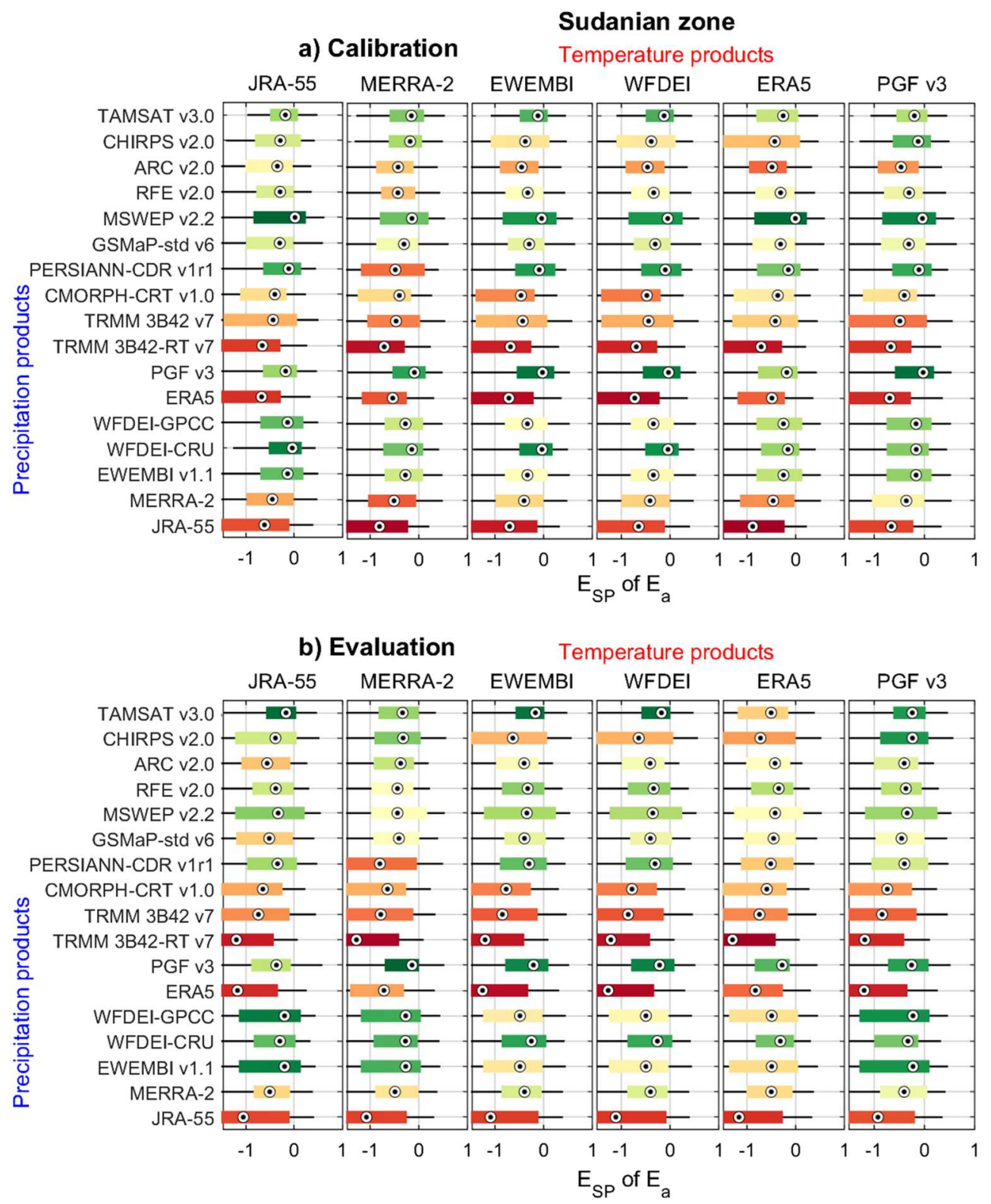

Figure S56. Spatial pattern efficiency $\left(E_{\mathrm{SP}}\right)$ of actual evaporation $\left(E_{\mathrm{a}}\right)$ over (a) the calibration (2003-2008) and (b) the evaluation (2009-2012) periods for the Sudanian zone, using different combinations of precipitation and temperature products for hydrological modelling. The boxplots are colored from the best (green) to the worst performance (red) based on the median value. 

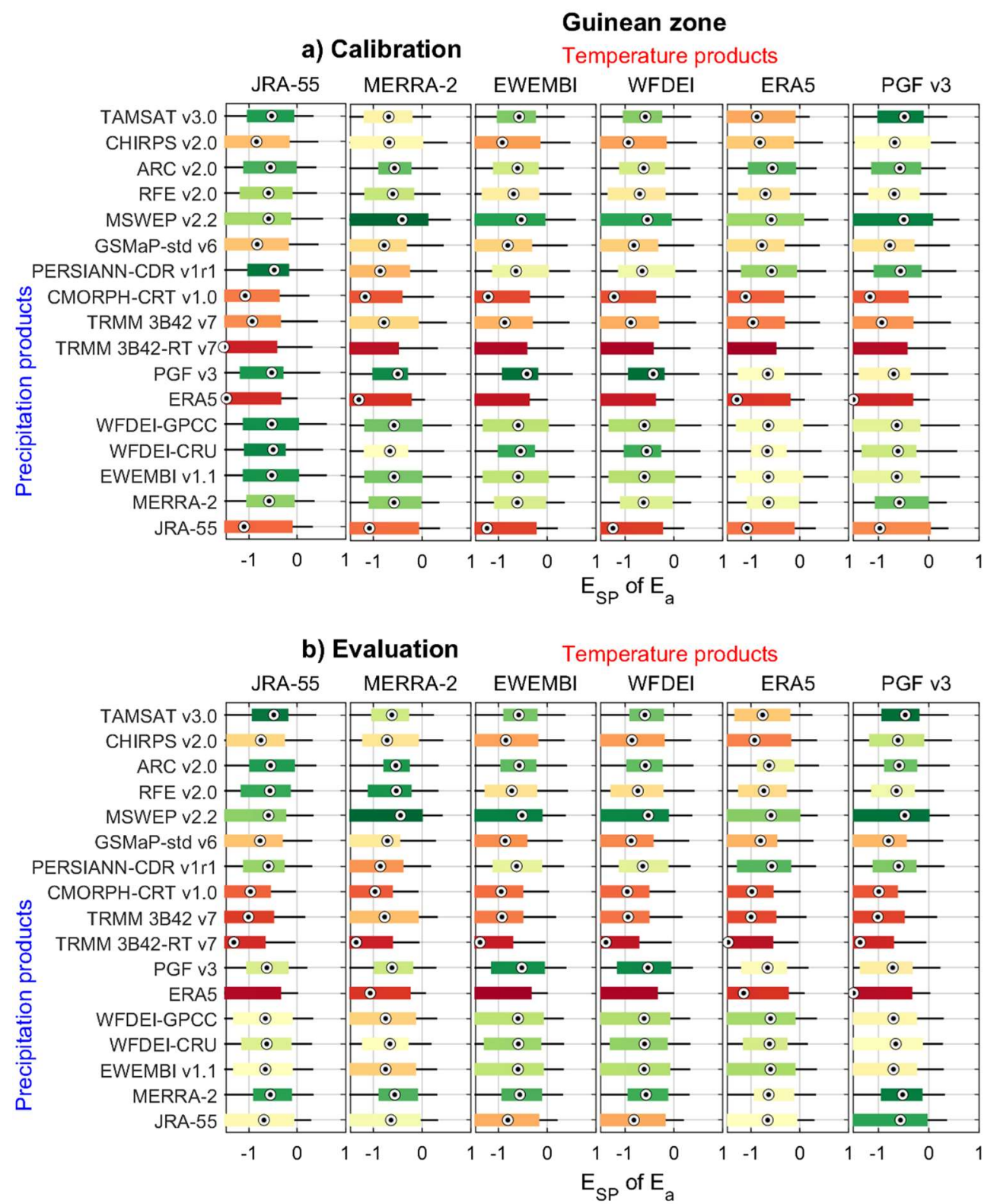

Figure S57. Spatial pattern efficiency $\left(E_{\mathrm{SP}}\right)$ of actual evaporation $\left(E_{\mathrm{a}}\right)$ over (a) the calibration (2003-2008) and (b) the evaluation (2009-2012) periods for the Guinean zone, using different combinations of precipitation and temperature products for hydrological modelling. The boxplots are colored from the best (green) to the worst performance (red) based on the median value. 
8.3 Time series

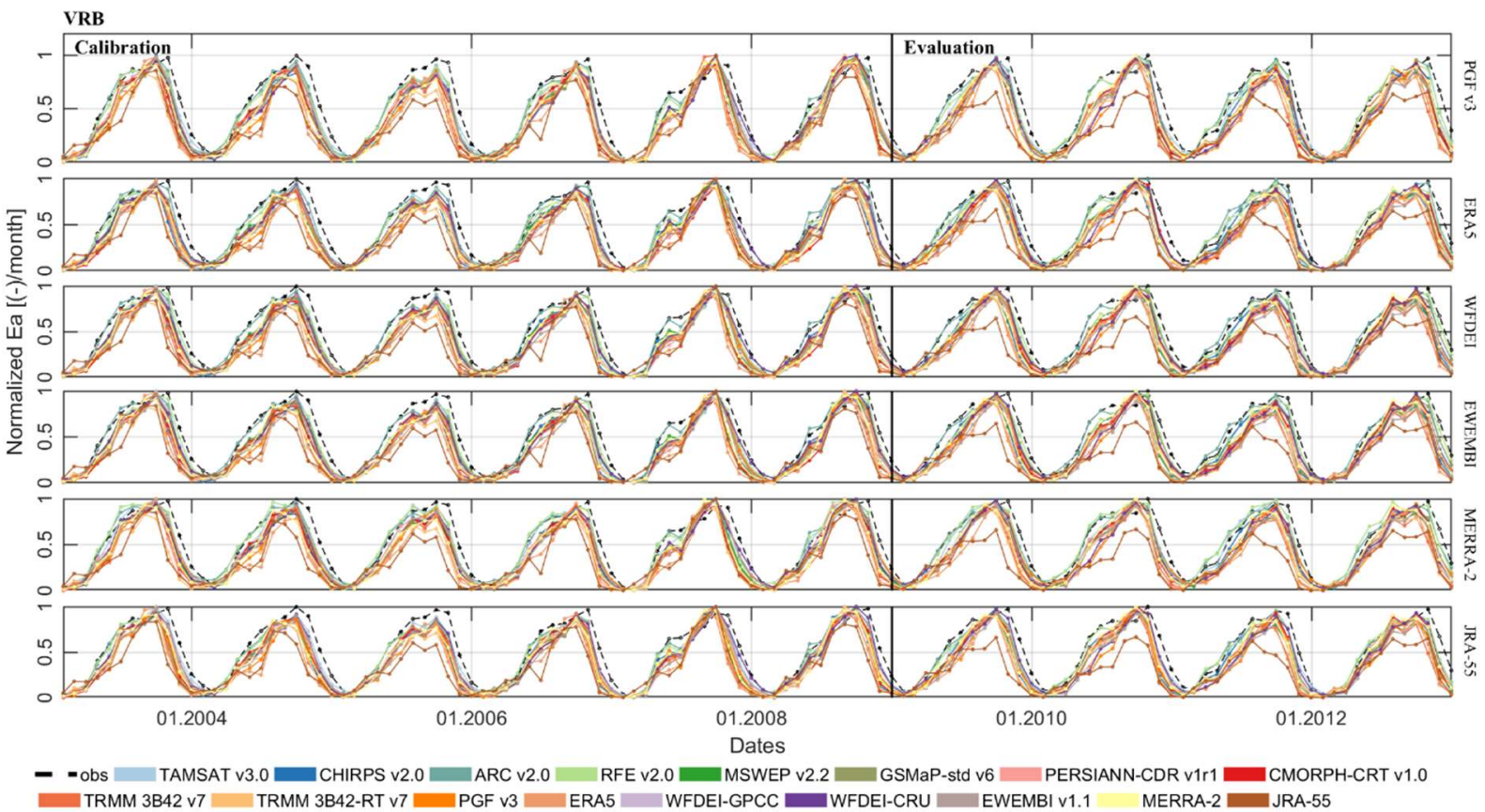

Figure S58. Monthly time series of actual evaporation $\left(E_{\mathrm{a}}\right)$ in the Volta River basin (VRB) obtained for different combinations of rainfall datasets (colors in legend) and temperature datasets (right y-axis of the 6 subplots) used as inputs in a hydrological model (i.e. mHM). All values are normalized between 0 and 1.

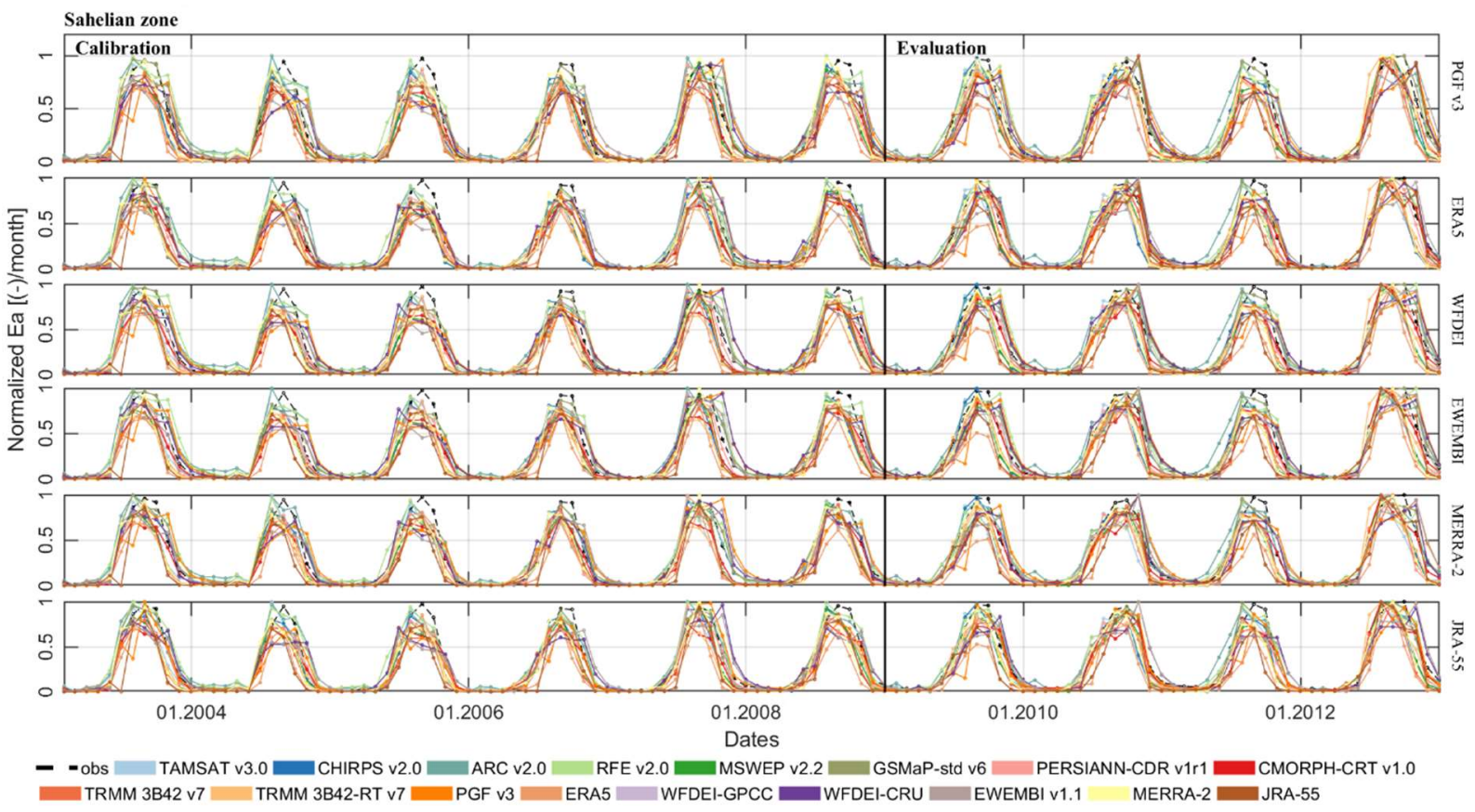

Figure S59. Monthly time series of actual evaporation $\left(E_{\mathrm{a}}\right)$ in the Sahelian zone of the Volta basin obtained for different combinations of rainfall datasets (colors in legend) and temperature datasets (right y-axis of the 6 subplots) used as inputs in a hydrological model (i.e. mHM). All values are normalized between 0 and 1. 


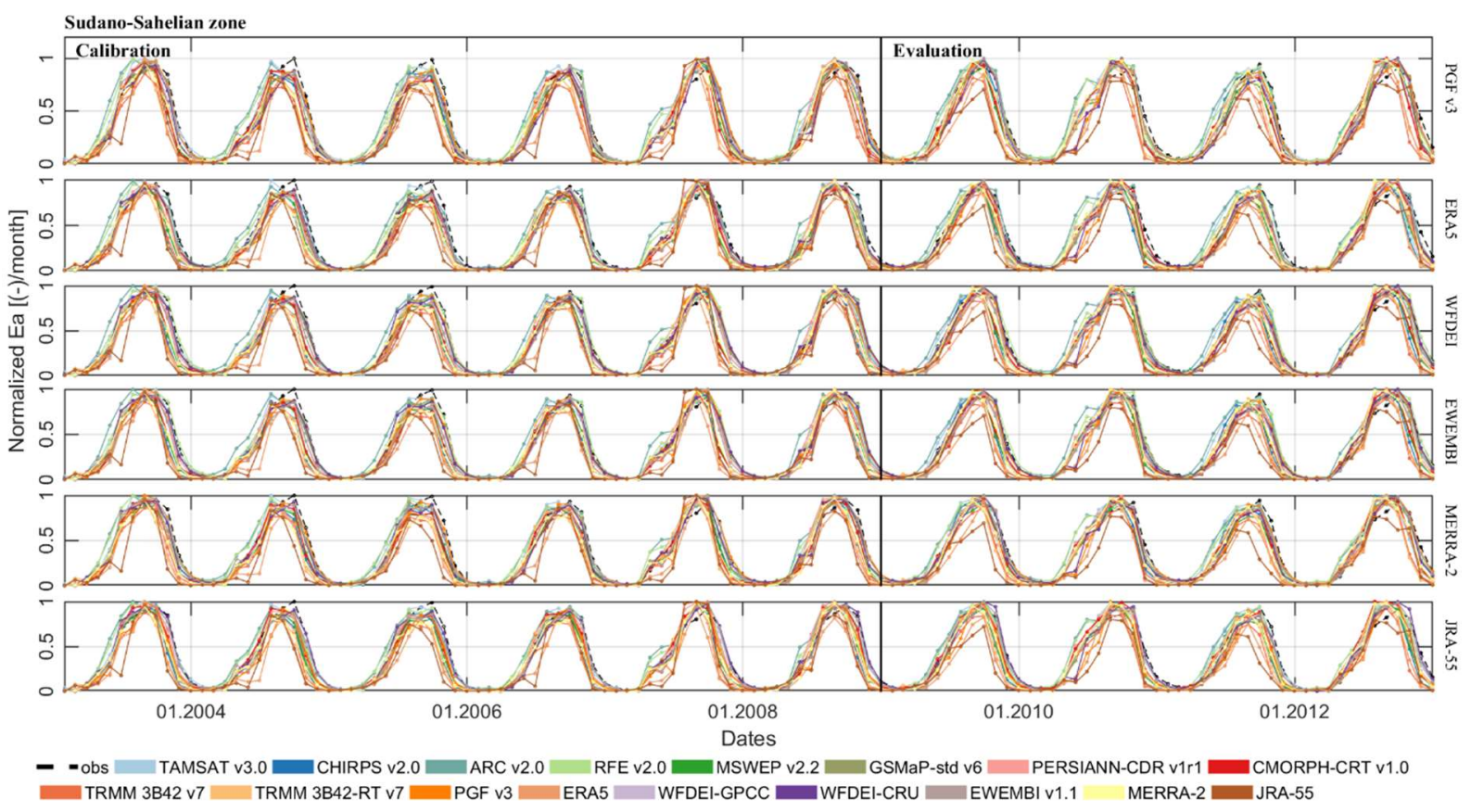

Figure S60. Monthly time series of actual evaporation $\left(E_{\mathrm{a}}\right)$ in the Sudano-Sahelian zone of the Volta basin obtained for different combinations of rainfall datasets (colors in legend) and temperature datasets (right y-axis of the 6 subplots) used as inputs in a hydrological model (i.e. mHM). All values are normalized between 0 and 1.

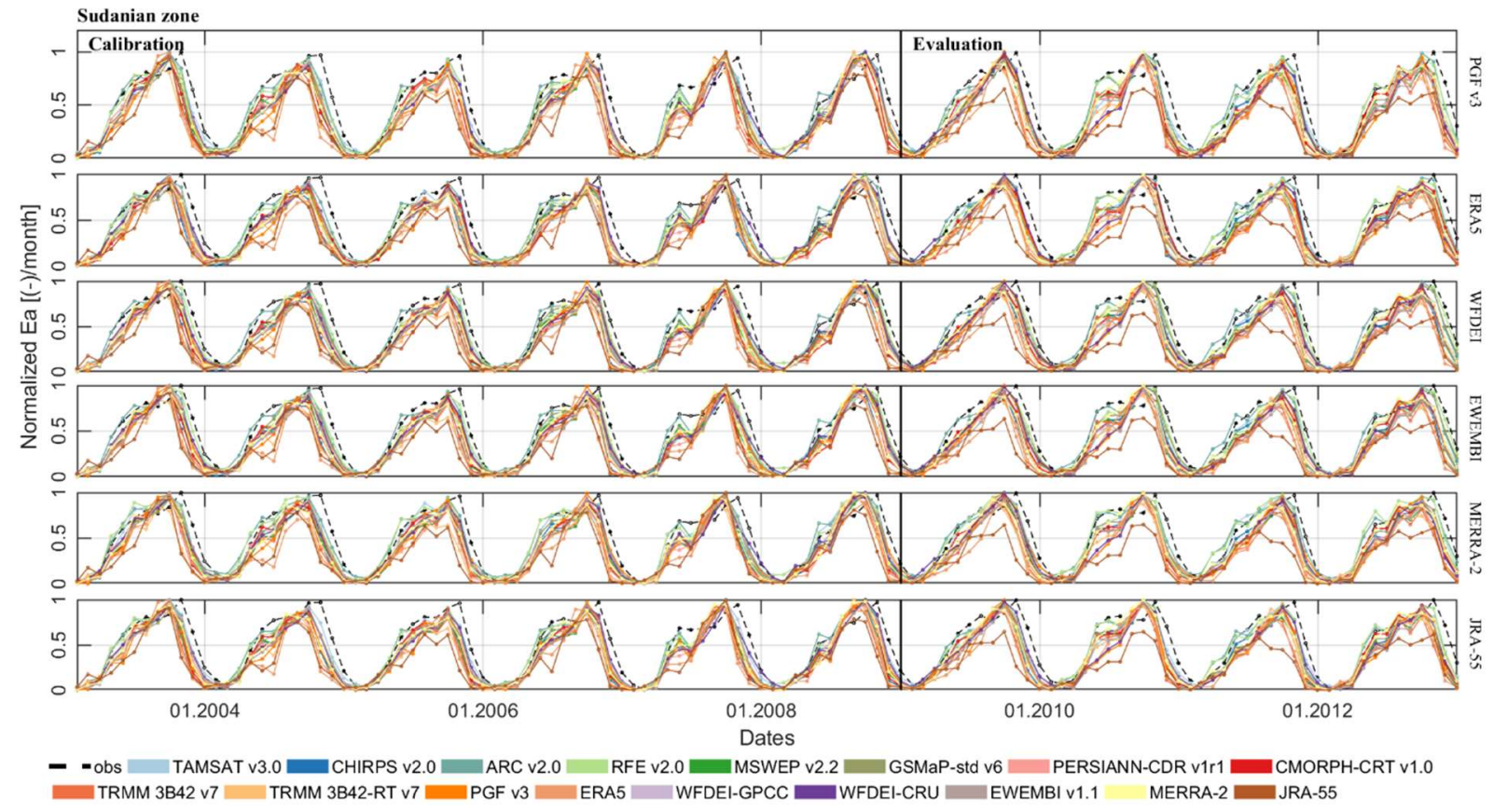

Figure S61. Monthly time series of actual evaporation $\left(E_{\mathrm{a}}\right)$ in the Sudanian zone of the Volta basin obtained for different combinations of rainfall datasets (colors in legend) and temperature datasets (right y-axis of the 6 subplots) used as inputs in a hydrological model (i.e. mHM). All values are normalized between 0 and 1 . 


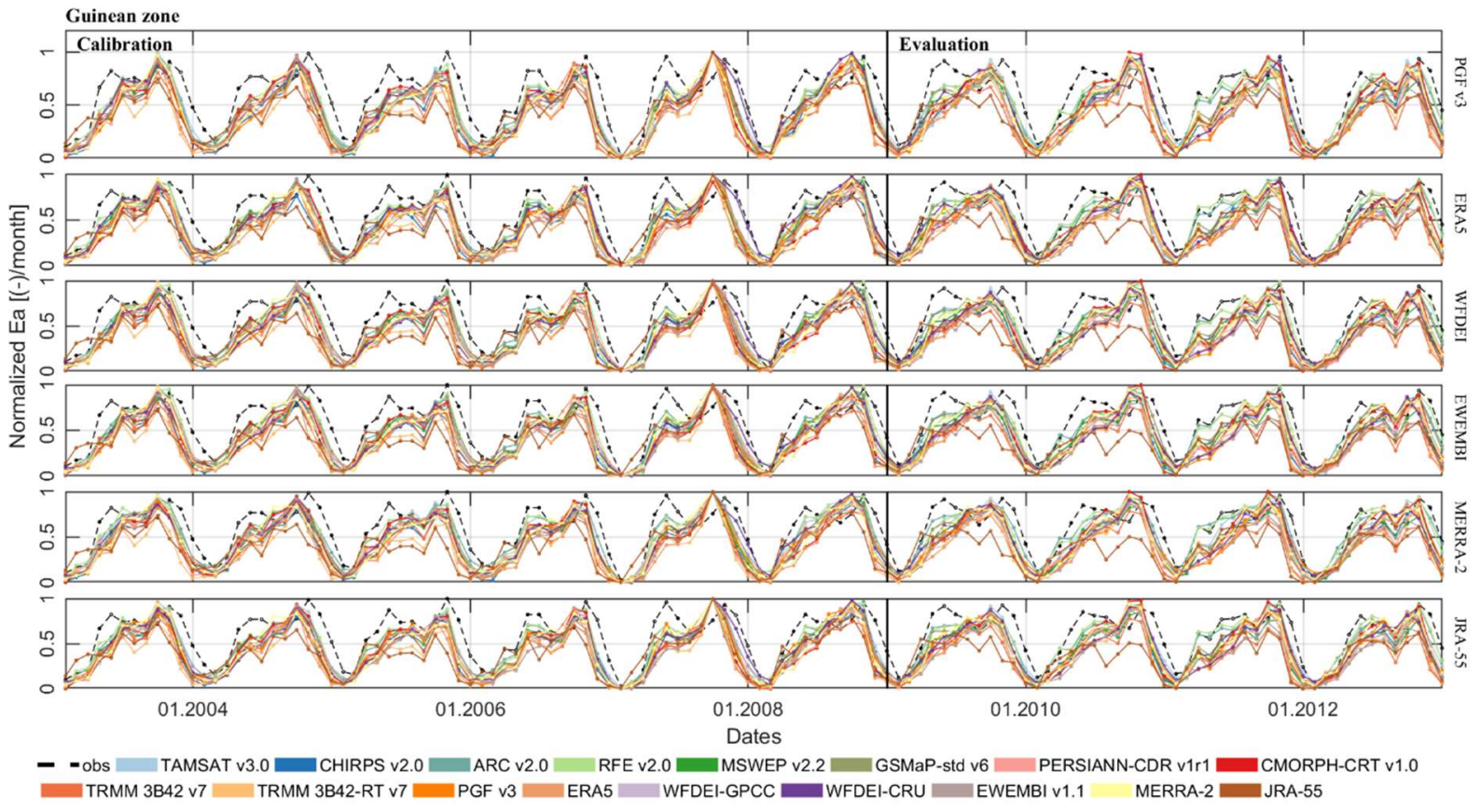

Figure S62. Monthly time series of actual evaporation $\left(E_{\mathrm{a}}\right)$ in the Guinean zone of the Volta basin obtained for different combinations of rainfall datasets (colors in legend) and temperature datasets (right y-axis of the 6 subplots) used as inputs in a hydrological model (i.e. mHM). All values are normalized between 0 and 1. 
8.4 Maps of long-term average

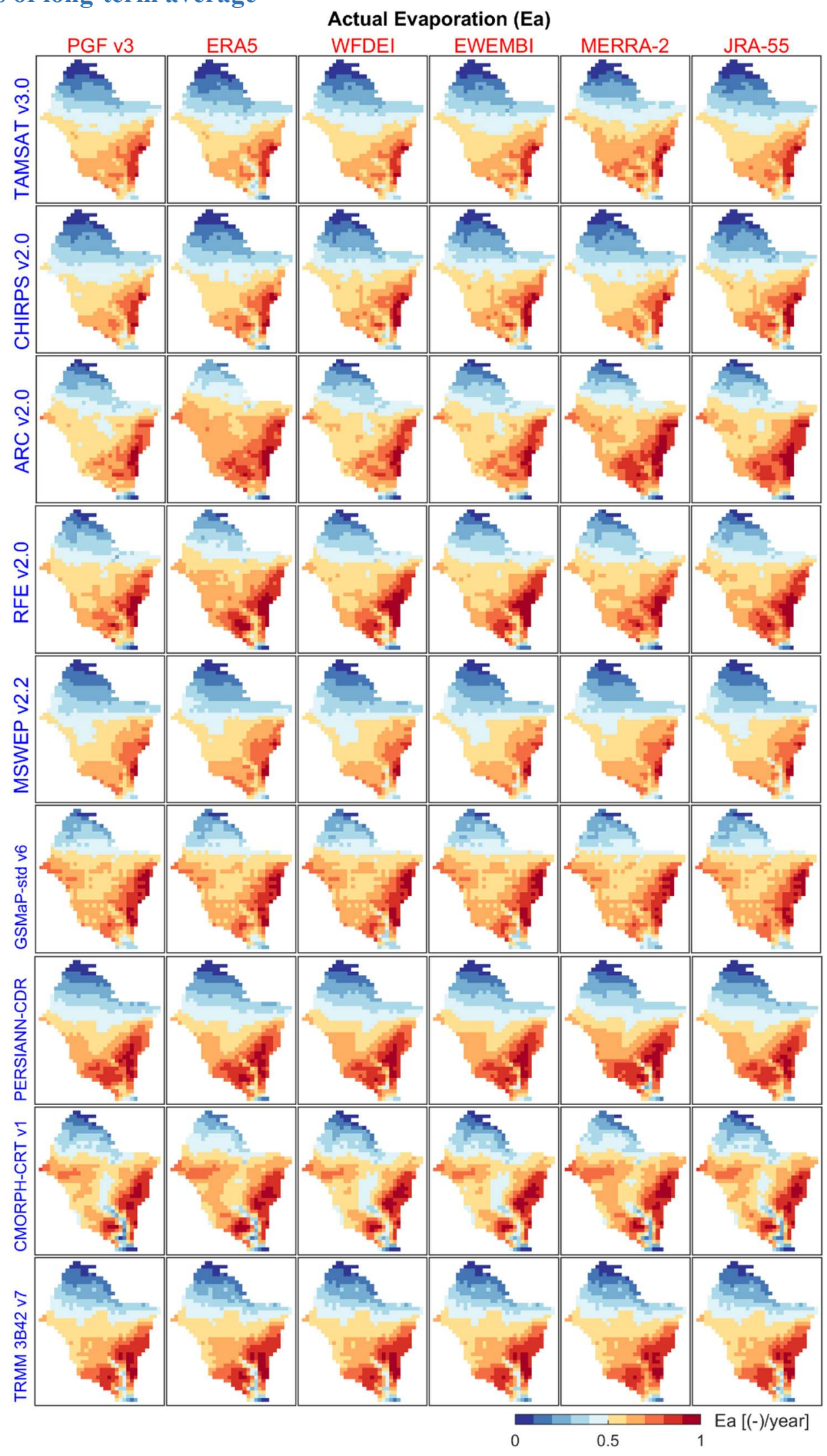

Figure S63. (Part1/2) Maps of long-term (2003-2012) annual average of actual evaporation $\left(E_{\mathrm{a}}\right)$ obtained as outputs of hydrological modelling using different combinations of rainfall datasets (y-axis, blue font) and temperature datasets ( $\mathrm{x}$-axis, red font). The values are normalized for better emphasizing on patterns and using a unique color scale. 

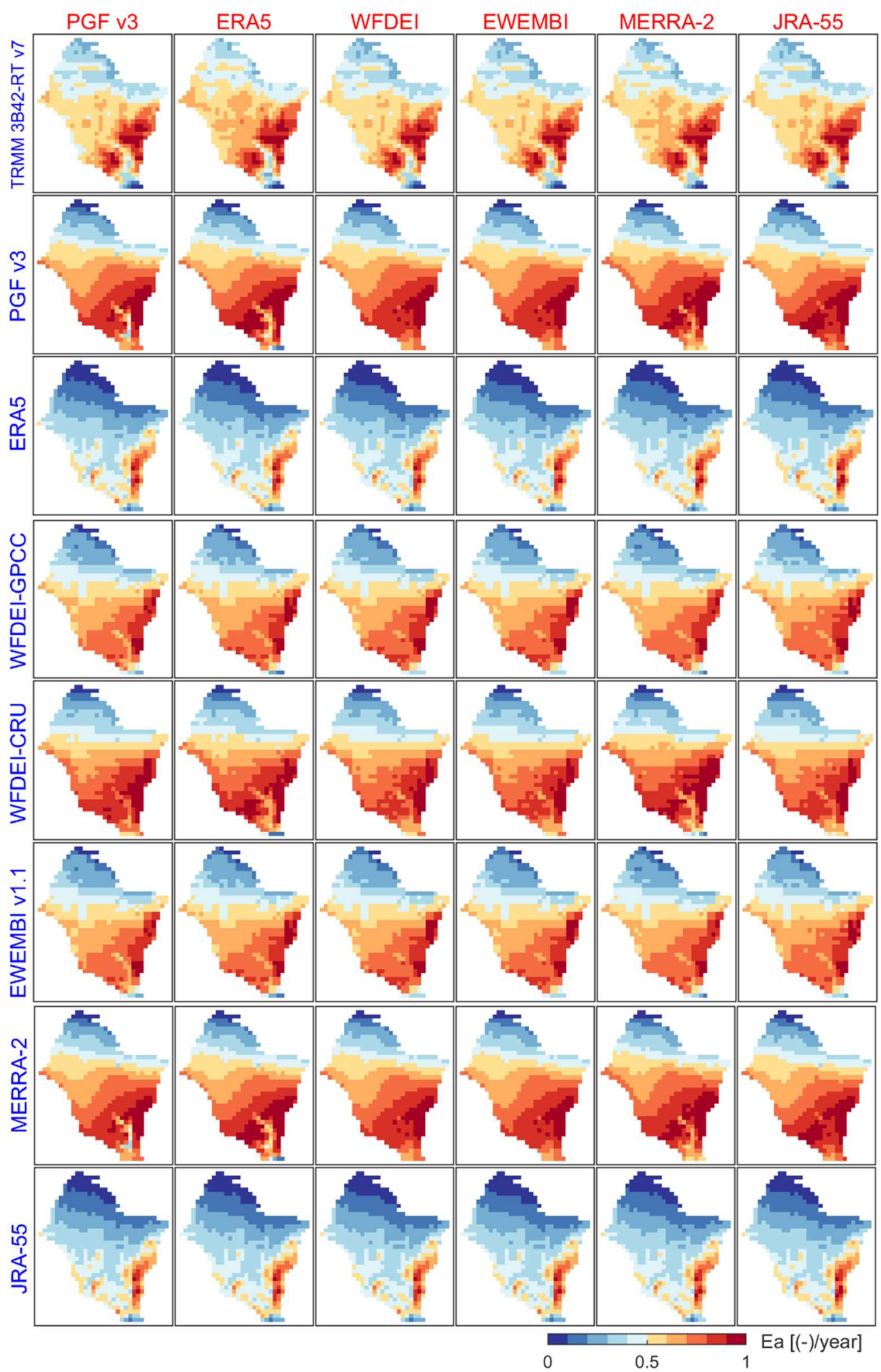

Figure S64. (Part2/2) Maps of long-term (2003-2012) annual average of actual evaporation $\left(E_{\mathrm{a}}\right)$ obtained as outputs of hydrological modelling using different combinations of rainfall datasets (y-axis, blue font) and temperature datasets (x-axis, red font). The values are normalized for better emphasizing on patterns and using a unique color scale. 


\section{Model parameters}

\subsection{Initial global parameter set used for model calibration}

Table S18. mHM global parameters. The description of the model parameters can be found in the work of Samaniego et al. (2010).

\begin{tabular}{|c|c|c|c|}
\hline Parameters & Lower bound & Upper bound & Initial value \\
\hline \multicolumn{4}{|l|}{ Interception } \\
\hline canopylnterceptionFactor & 0.01 & 0.40 & 0.278 \\
\hline \multicolumn{4}{|l|}{ Soil Moisture } \\
\hline orgMatterContent_forest & 0.00 & 200.00 & 105.84 \\
\hline orgMatterContent_impervious & 0.00 & 1.00 & 0.755 \\
\hline orgMatterContent_pervious & 0.00 & 4.00 & 2.967 \\
\hline PTF_lower66_5_constant & 0.65 & 1.00 & 0.939 \\
\hline PTF_lower66_5_clay & 0.000 & 0.004 & 0.003 \\
\hline PTF_lower66_5_Db & -0.373 & -0.187 & -0.204 \\
\hline PTF_higher66_5_constant & 0.536 & 1.123 & 0.924 \\
\hline PTF_higher66_5_clay & -0.009 & 0.005 & -0.001 \\
\hline PTF_higher66_5_Db & -0.551 & -0.091 & -0.107 \\
\hline PTF_Ks_constant & -1.700 & -0.285 & -0.424 \\
\hline PTF_Ks_sand & 0.0060 & 0.0260 & 0.0064 \\
\hline PTF_Ks_clay & 0.001 & 0.013 & 0.005 \\
\hline rootFractionCoefficient_forest & 0.800 & 0.999 & 0.864 \\
\hline rootFractionCoefficient_impervious & 0.800 & 0.950 & 0.912 \\
\hline rootFractionCoefficient_pervious & 0.001 & 0.090 & 0.022 \\
\hline infiltrationShapeFactor & 1.000 & 4.000 & 3.945 \\
\hline \multicolumn{4}{|l|}{ Direct sealed area runoff } \\
\hline imperviousStorageCapacity & 0.00 & 100.00 & 5.340 \\
\hline \multicolumn{4}{|l|}{ Potential evapotranspiration } \\
\hline PET_a_forest & 0.30 & 1.30 & 0.5282 \\
\hline PET_a_impervious & 0.90 & 1.30 & 0.9743 \\
\hline PET_a_pervious & 0.30 & 1.30 & 0.5088 \\
\hline PET_b & 0.00 & 1.50 & 1.4993 \\
\hline PET_c & -3.00 & 0.00 & -1.0330 \\
\hline HargreavesSamaniCoeff & 0.0020 & 0.0027 & 0.0023 \\
\hline \multicolumn{4}{|l|}{ Interflow } \\
\hline interflowStorageCapacityFactor & 75.00 & 400.00 & 313.85 \\
\hline interflowRecession_slope & 0.00 & 10.00 & 5.51 \\
\hline fastInterflowRecession_forest & 0.00 & 3.00 & 2.52 \\
\hline slowlnterflowRecession_Ks & 1.00 & 30.00 & 22.21 \\
\hline exponentSlowinterflow & 0.005 & 0.300 & 0.283 \\
\hline \multicolumn{4}{|l|}{ Percolation } \\
\hline rechargeCoefficient & 0.00 & 200.00 & 183.51 \\
\hline \multicolumn{4}{|l|}{ Routing } \\
\hline streamflow_celerity & 0.10 & 15.00 & 12.47 \\
\hline \multicolumn{4}{|l|}{ Geology } \\
\hline GeoParam1 & 1.00 & 1500.00 & 1037.16 \\
\hline GeoParam2 & 1.00 & 1500.00 & 709.99 \\
\hline GeoParam3 & 1.00 & 1500.00 & 381.09 \\
\hline GeoParam4 & 1.00 & 1500.00 & 525.30 \\
\hline GeoParam5 & 1.00 & 1500.00 & 783.83 \\
\hline
\end{tabular}


9.2 Distribution of model parameters after model calibration

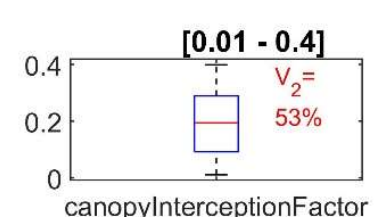

canopyInterceptionFactor
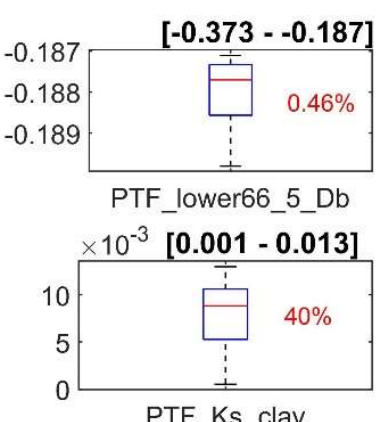

PTF_Ks_clay

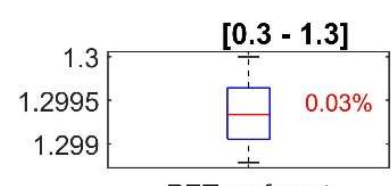

PET_a_forest

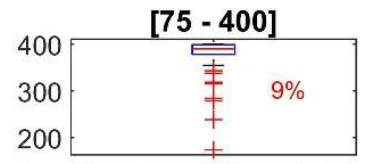

interflowStorageCapacityFactor

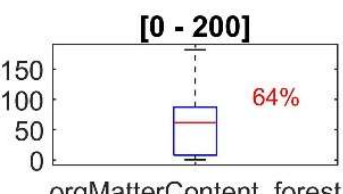

orgMatterContent forest
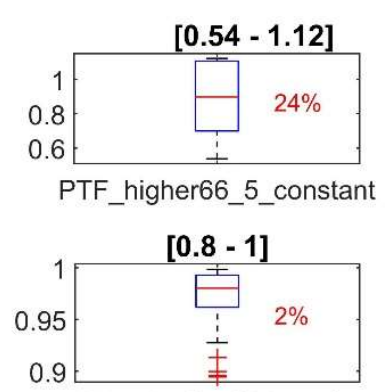

rootFractionCoefficient for

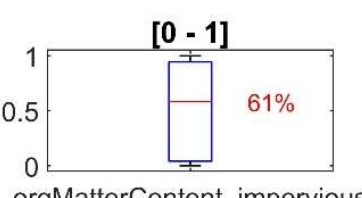

orgMatterContent_impervious

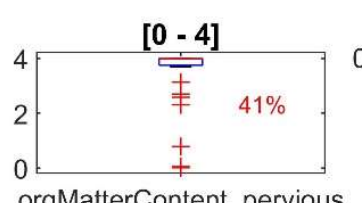

$\times 10^{-3}[-0.009-0.005]$
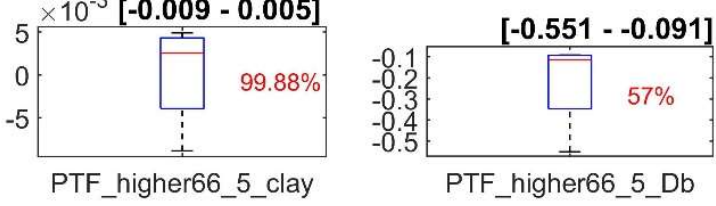

PTF_higher66_5_Db
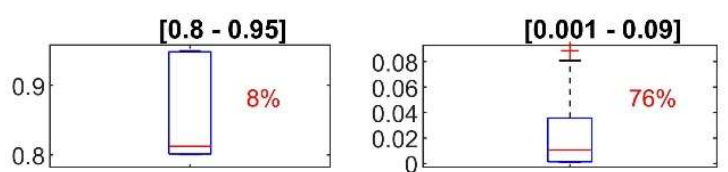

[0.9 - 1.3]

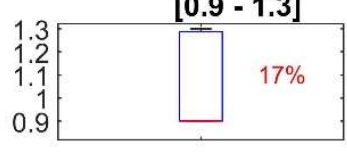

PET_a impervious

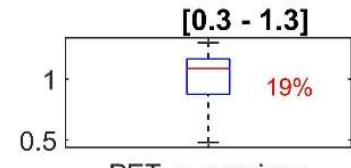

PET_a_pervious

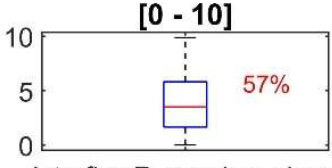

interflowRecession_slope

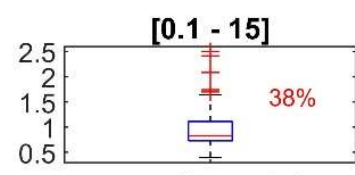

streamflow_celerity

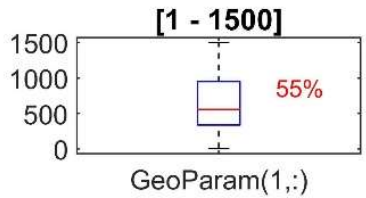

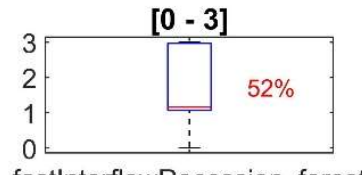

fastInterflowRecession forest

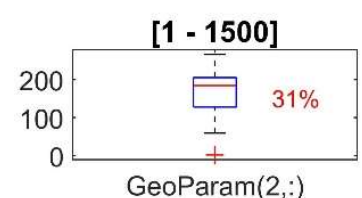

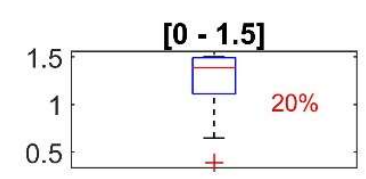

PET_b

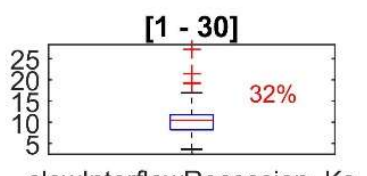

slowInterflowRecession Ks

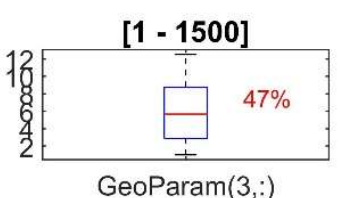

PTF_Ks_constant

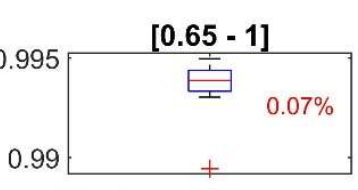

PTF lower66 5 constant
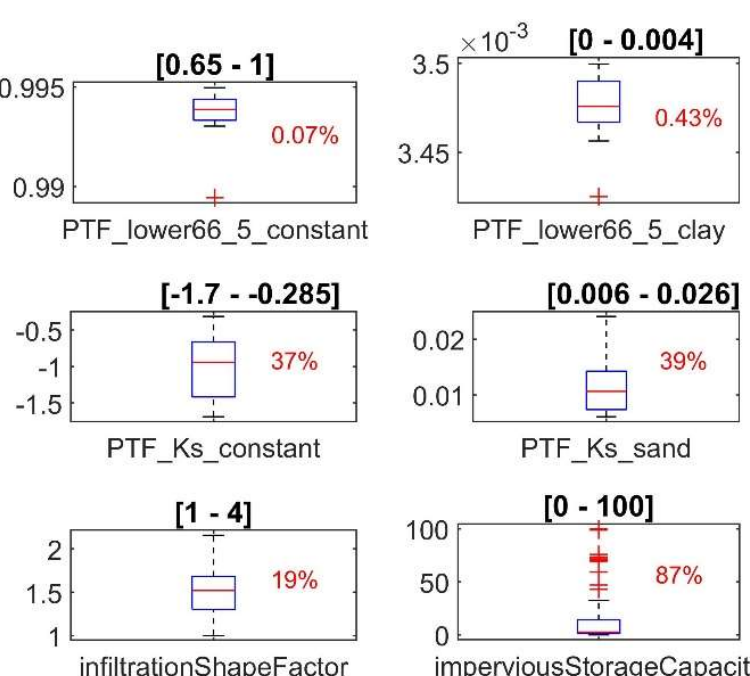

$[-3-0]$

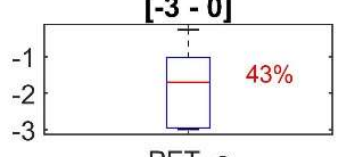

PET C
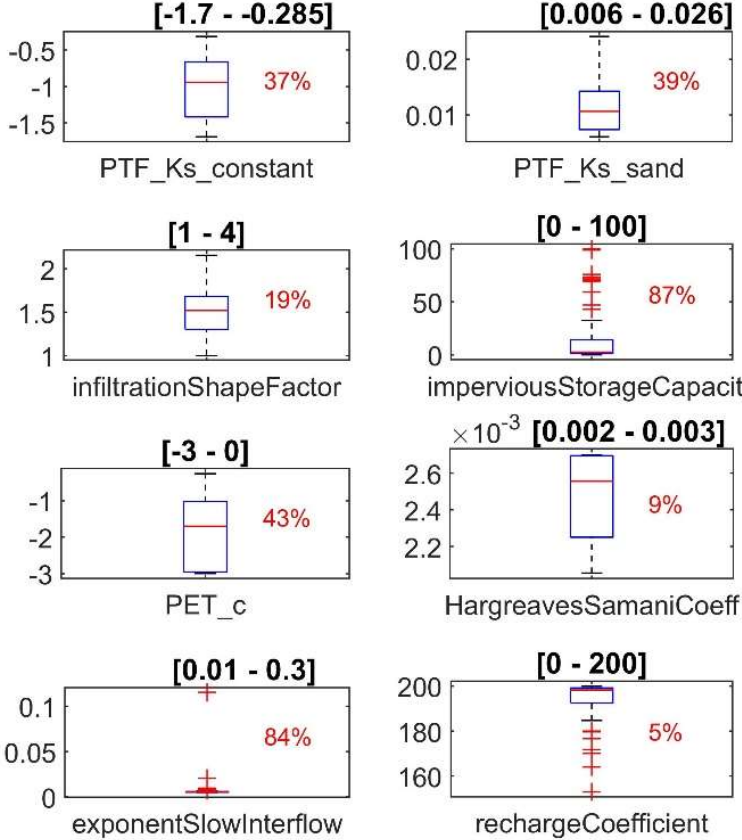

PTF_Ks_sand

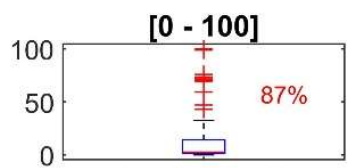

imperviousStorageCapacity

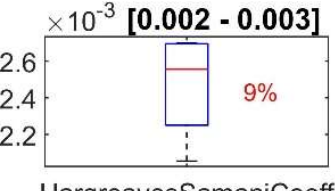

HargreavesSamaniCoeff
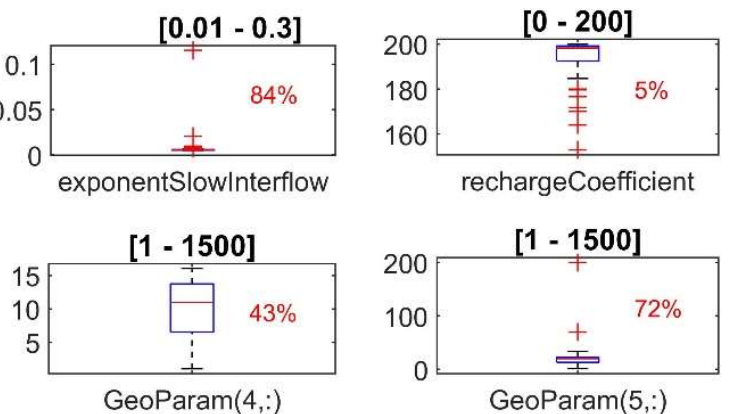

Figure S65: Distribution of mHM global parameters after model calibration with different combinations of precipitation and temperature datasets as inputs. Each boxplot has 102 elements corresponding to parameter values obtained with different input datasets. The initial parameters' ranges are provided in squared brackets. The second-order coefficient of variation $\left(V_{2}\right)$ is given in percentage. 

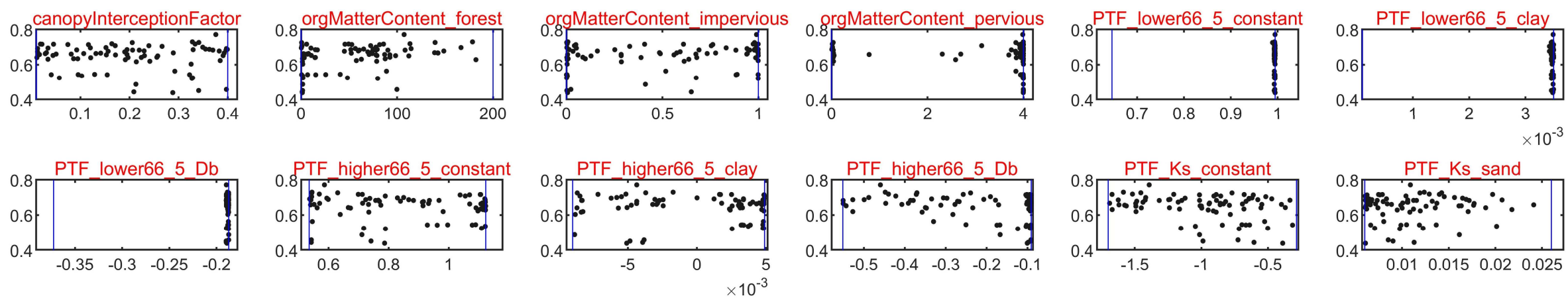

PTF_Ks_sand
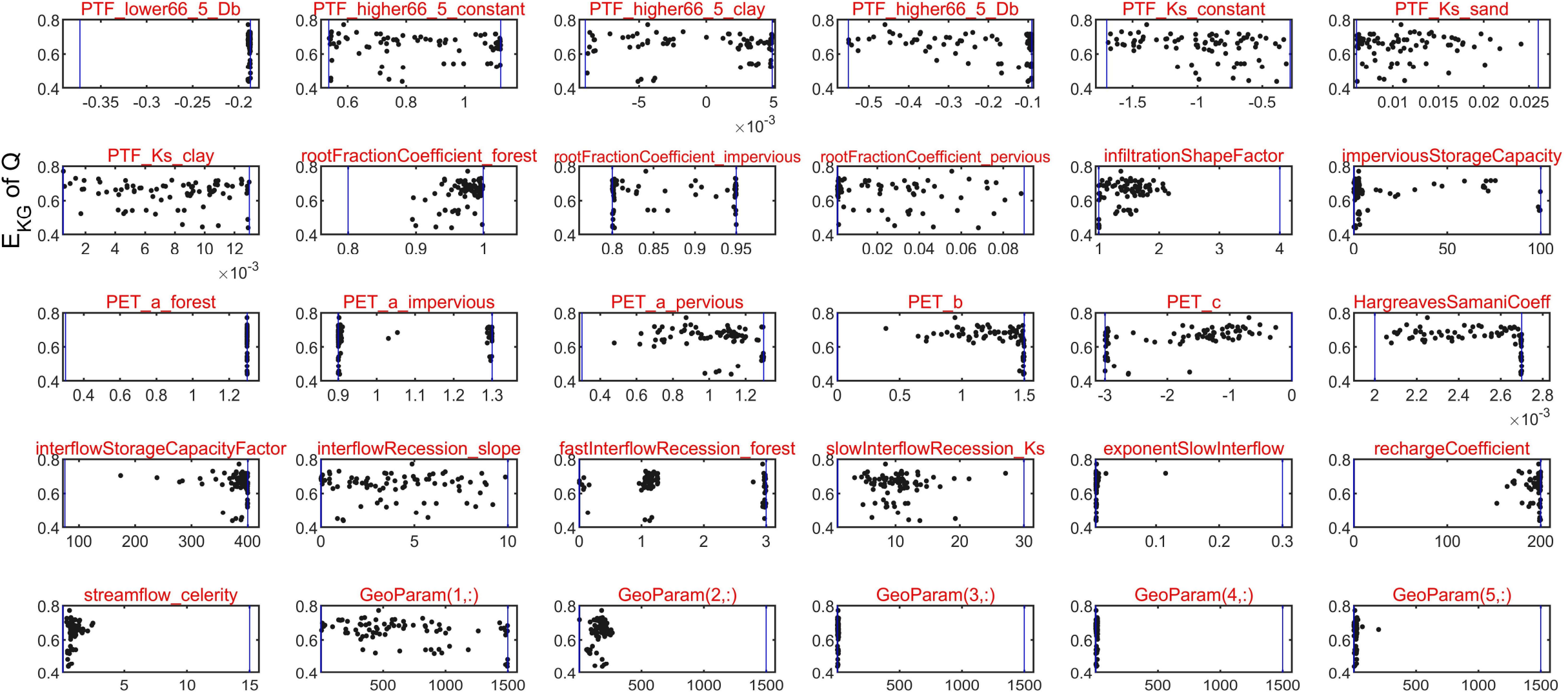

Figure S66: Model performance for streamflow $(Q)$ as a function of final global parameters (subplot titles) obtained after model calibration with different combinations of precipitation and temperature datasets as inputs. The x-axis give the parameter values for 102 different input datasets. The y-axis gives the Kling-Gupta efficiency ( $E K G)$ of $Q$ over the simulation period (2003-2012). The vertical lines (in blue) show the lower and upper bounds of the parameter ranges. 